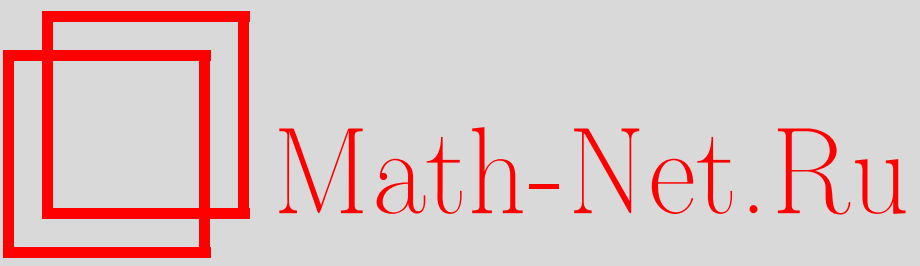

В. А. Малышев, Гиббсовские и квантовые дискретные пространства, УМН, 2001, том 56, выпуск 5, 117-172

DOI: https://doi.org/10.4213/rm402

Использование Общероссийского математического портала Math-Net.Ru подразумевает, что вы прочитали и согласны с пользовательским соглашением

http://www.mathnet.ru/rus/agreement

Параметры загрузки:

IP : 54.174 .149 .18

26 апреля 2023 г., 12:52:08 


\title{
ГИББСОВСКИЕ И КВАНТОВЫЕ ДИСКРЕТНЫЕ ПРОСТРАНСТВА
}

\author{
В. А. МАЛЫШЕВ
}

Гиббсовское поле является одним из центральных объектов теории вероятностей, математической статистической физики и евклидовой теории поля. В статье определяется и изучается естественное его обобшение, когда пространство (решетка, граф̆), на котором определено случайное поле, само является случайным. Более того, эта случайность не задана априори и независимо от конфигурации, но пространство и конфигурация на нем зависят друг от друга, и оба задаются гиббсовской процедурой. Полученньй объект назван гиббсовским семейством потому, что он параметризует гиббсовские поля на различных граффах, входящих в носитель распределения. Мы исследуем также квантовый аналог гиббсовских семейств. Обсуждаются связи с современной теорией струн и квантовой гравитацией.

Библиография: 55 названий.

\section{СОДЕРЖАНИЕ}

1. Введение

2. Гиббсовские семейства .............................................. 120

2.1. Конечные гиббсовские семейства ............................... 120

2.2. Предельные гиббсовские семейства . . . . . . . . . . . . . . . . . . . . . 126

2.3. Эмпирические распределения ............................... 128

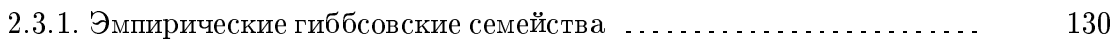

2.3.2. Эмпирические распределения в планарной гравитации . . . . . . . . 130

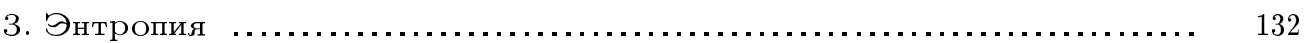

3.1. Произволшный род в размерности 2 . . . . . . . . . . . . . . . . . . . . 132

3.2. Размерности 3 и выше . . . . . . . . . . . . . . . . . . . . . . . . . . . . 137

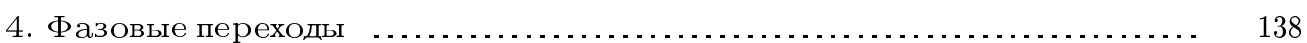

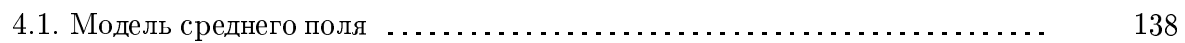

4.2. Фазовые переходы в модели с границами

4.2.1. Связь с топологической теорией поля …................... 143

4.3. Неединственность в модели с низкой энтропией … . . . . . . . . . . . . . . . . . . 145

4.4. Гиббсовская характеризация структур ....................... 147

4.4.1. Единственность в модели с высокой энтропией . . . . . . . . . . . . . . . 147

4.4.2. Гиббсовские многообразия групп ....................... 148

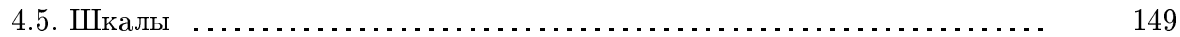

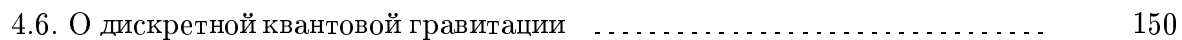


5. Квантовые дискретные пространства ............................. 156

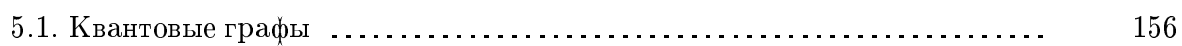

5.1.1. $C^{*}$-алгебры ........................................... 159

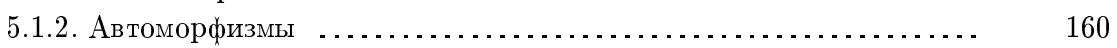

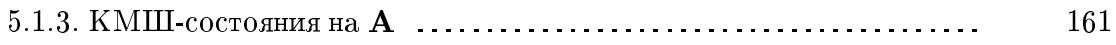

5.2. Примеры и структура гамильтонианов ................................ 164

5.2.1. Квантование по Тоофту ................................. 164

5.2.2. Квантовые спиновые системы на фиксированном графе ....... 165

5.2.3. Линейные грамматики и теплицевы операторы $\ldots . . . . . . . . . . .2165$

5.2.4. Расширение и сжатие пространства . . . . . . . . . . . . . . . . . . . . . . 167

5.2.5. Лоренцевы модели и грамматики ......................... 168

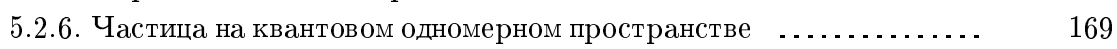

5.2.7. Два типа квантов пространства ............................. 169

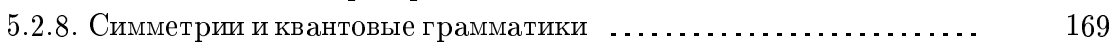

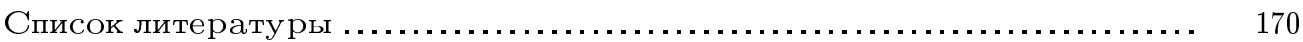

\section{1. Введение}

Мы перечисляем различные мотивации, которые привели к данной работе.

Гиббсовское поле [1], [2] является одним из центральных объектов теории вероятностей, математической статистической физики и евклидовой теории поля [3]. Здесь определяется естественное его обобшение, когда пространство (решетка, граф)), на котором определено случайное поле, само является случайным. Более того, эта случайность не задана априори и независимо от конфигурации, но пространство и конфигурация на нем зависят друг от друга, и задаются оба гиббсовской процедурой. Даже если на графе нет конфигурации, получается нетривиальньй объект, которьй здесь назван гиббсовским графом. Полученный объект назван гиббсовским семейством потому, что он параметризует конечное или континуальное множество пар $(G, \mu)$, где $G$-графф, $\mu$-гиббсовское поле на $G$, входящих в носитель распределения.

Квантовый аналог гиббсовских семейств является обобшением квантовых спиновых систем [4] на случай квантовой решетки. Даже если нет спина, полученньй объект - квантовое дискретное пространство - интересен с многих точек зрения.

Мы излагаем здесь математические основы такой теории, включающей в себя различные аспекты - от общего понятия локальности до комбинаторики графов.

Другой мотивацией данной статьи являлись вопросы, возникающие при обдумьвании общих аспектов более современных физических теорий, таких как теория струн, $M$-теория, другие подходы к квантовой гравитации. Первый вопрос возникает в связи с разньми аспектами локальности. Пространство в физических теориях задается обычно априори как сцена для всех дальнейших действий. Долгое время только трехмерное евклидово пространство изучалось в физике. В XX веке стала варьироваться размерность и от глобально евклидова пространства перешли к многообразиям. При этом глобальная структура многообразия определяется вешеством согласно уравнениям общей теории относительности, ограничениям из теории струн и т. д. Тем не менее постулат локальной гладкости остался и, в том или ином виде, сохраняется и теперь. Однако этот постулат серьезно расшатьвается расходимостями квантовой теории поля, квантовостью метрики и т. д. Логика вешей ведет к естественным вопросам о том, что может быть заменой локальной евклидовости. В настоящее время 
многие считают, что ответ надо искать в понятии дискретного пространства. Оказывается однако, что для дискретных пространств само понятие локальности допускает различные толкования, которые здесь обсуждаются.

С другой стороны, пространство приобретает равный статус с полями. Другими словами, оно само должно быть случайным или квантовым. Заметим, что понятие квантового дискретного пространства часто звучит в связи с некоммутативной геометрией [5]-[8]. Здесь не исследуется эта связь, но делается акцент на аспекте квантовых пространств, связанных с локальностью. Локальность в физике ассоциируется прежде всего с гиббсовостью. Пространство строится из дискретных элементов (квантов) особым (гиббсовским) способом. Если допустить, что окрестность точки может содержать сколько угодно квантов, то при таком общем понимании локальности, чтобы получить известные пространства, необходима процедура перенормировок, аналогичная перенормировкам в квантовой теории поля. Если же понимать локальность в метрическом смысле, т.е. если квант соответствует всей окрестности, то перенормировки не нужны. Последнее понимание не вполне удовлетворительно, так как сама метрика не должна быть фиксирована.

План статьи таков. В разделе 2.1 мы вводим два определения конечных гиббсовских дискретных пространств (конечные гиббсовские семейства) - локальное (где энергия равна сумме энергий подграфов) и суперлокальное (где энергия есть сумма энергий всех окрестностей заданного радиуса). Это соответствует разному пониманию локальности в случае отсутствия фиксированного пространства. Показьвается, что эти два определения могут быть сведены друг к другу только с помощью процедуры, напоминающей перенормировки в квантовой теории поля. В разделе 2.2 рассматриваются предельные гиббсовские семейства, находится аналог условия Добрушина-Лэнфорда-Рюэлля, условие компактности. Показывается, что для заданного гиббсовского семейства условная мера на конфигурациях, при условии, что граф фиксирован, является гиббсовской мерой в обычном смысле с тем же потенциалом. В разделе 2.3 предложен аналог вероятностных мер на множестве счетных графов без нумерации вершин и без выделенной вершины, где колмогоровский метод конечномерных распределений не работает. Этот аналог назван эмпирическим распределением, приводятся примеры эмпирических гиббсовских распределений, возникаюших из дискретной квантовой гравитации.

В разделе 3 мы получаем логарифмическую асимптотику $\sum_{g} F_{b, p}(g)$, где $F_{b, p}(g)-$ число карт с $p+1$ вершиной и $b+p$ ребрами на замкнутой поверхности рода $g$. Частным случаем карты является триангуляция, и опыт подсчета числа карт на поверхности фиксированного рода показывает одинаковый характер асимптотики вне зависимости от рассматриваемого класса карт.

В разделе 4.2 подробно изучена простейшая модель дискретной планарной квантовой гравитации (с границей), в которой есть полньй контроль: существование термодинамического предела, явный вид свободной энергии, полная фазовая диаграмма с тремя разными фазами, включая критическую точку. Доказьвается также эффект влияния наблюдателя на флуктуацию длины границы в критической точке. Соответствующая модель с двумя гранищами изучена только в “высокотемпературной” области. В разделе 4.3 приводится пример топологического фазового перехода в локально однородном графе, где 1-окрестности всех вершин одинаковы. В разделе 4.4 доказывается единственность предельного гиббсовского семейства в модели с факториально 
растущей статсуммой. Это же позволяет дать локальную гиббсовскую характеризацию деревьев и свободных некоммутативных групп. В разделе 4.5 мы обсуждаем как существенно разные топологии могут возникать на разных шкалах. Понятие шкалыболее общая альтернатива термодинамическому предельному переходу.

В разделе 5.1 вводятся квантовые аналоги классических гиббсовских графов с локальной структурой - квантовые грамматики на графах. Доказьваются основные утверждения о самосопряженности гамильтониана, существовании группы автоморфизмов соответствующих $C^{*}$-алгебр. Квантовые спиновые системы являются частным случаем этих конструкций. Для линейных графов и высоких температур доказывается сушествование и аналитичность состояний Кубо-Мартина-Швингера. В разделе 5.2 приводятся разнообразные примеры $C^{*}$-алгебр и гамильтонианов в квантовых грамматиках: линейные грамматики и теплицевы операторы, квантовое расширение и сжатие пространства, двумерная лоренщева модель и др.

\section{2. Гиббсовские семейства}

\section{1. Конечные гиббсовские семейства.}

Спиновые графы. Мы рассматриваем графы $G$ (конечные или счетные) с множеством $V=V(G)$ вершин и множеством $L=L(G)$ ребер. Будет всегда предполагаться, что между двумя вершинами не более одного ребра и что каждая вершина имеет конечную степень (число инщидентных ей ребер). Графы предполагаются связными, если не оговорено противное.

Если не оговорено противное, предполагается отсутствие петель. Однако обшие результаты легко переносятся на графы с петлями и с кратными ребрами. Если рассматриваются такие более обшие графы, то петля прибавляет к степени вершины (число инщидентных ей ребер) число 2.

Подграф графа $G$ определяется подмножеством $V_{1} \subset V$ вершин вместе с некоторыми ребрами, унаследованными из $L$ и соединяюшими вершины из $V_{1}$. Подграф $G\left(V_{1}\right)$ графа $G$ с множеством вершин $V_{1}$ назьвается регулярным, если ему принадлежит любое ребро из $L(G)$ между вершинами из $V_{1}$.

Множество $V$ вершин образует метрическое пространство, при этом расстояние $d(x, y)$ между вершинами $x, y \in V$ определяется как минимум длин путей, соединяюших эти вершины. Длина ребра полагается равной 1.

Спинграф̆ $\alpha=(G, s)$ определяется как граф̆ $G$ вместе с функцией $s: V \rightarrow S$, где $S-$ некоторое множество (значений спина). Графы и спинграфы всегда рассматриваются с точностью до изоморфизма, т.е. незанумерованные. Изоморфизм есть взаимно однозначное соответствие между множествами вершин, уважающее ребра и спины. Обозначим через $\mathscr{G}_{N}\left(\mathscr{A}_{N}\right)$ множество классов эквивалентности (относительно изоморфизма) связных графов (спинграфов) с $N$ вершинами. Счетные графы соответствуют $N=\infty$.

Далее, где это не вызовет неясностей, мы допускаем некоторую свободу терминологии: говорим о спинграфе или графе вместо класса эквивалентности, о подграфе вместо подспинграфа и т. д.

Корневые спинграфы. Мы будем также рассматривать графы с выделенной вершиной (корневые графы), назьваемой началом координат или корнем. В этом случае рассматриваются только изоморфизмы, сохраняющие начало координат. Когда 
надо подчеркнуть, что графы рассматриваются с выделенной вершиной $v$, мы будем писать $G^{(v)}, \mathscr{G}_{N}^{(v)}$ и т. д.

Определим кольцевую окрестность $\gamma(\alpha, v ; a, b)$ вершины $v$ как регулярньй подграф спинграфа $\alpha$ с множеством вершин $V(\gamma(\alpha, v ; a, b))=\left\{v^{\prime}: a \leqslant d\left(v, v^{\prime}\right) \leqslant b\right\} . O_{d}(v)=$ $\gamma(\alpha, v ; 0, d)$ называется $d$-окрестностью $v, O(v)=O_{1}(v) . R_{0}(G)=\max _{v \in V(G)} d(0, v)$ называется радиусом графа $G$ по отношению к вершине 0 . В частности, $R_{v}(O(v))=1$. Тогда диаметр графа $G$ равен $d(G)=\max _{v, v^{\prime} \in V(G)} d\left(v, v^{\prime}\right)=\max _{v \in V(G)} R_{v}(G)$.

Обозначим через $\mathscr{G}_{N}^{(0)}\left(\mathscr{A}_{N}^{(0)}\right)$ множество классов эквивалентности (относительно изоморфизма) связных графов (спинграфов) с корнем 0 и радиусом $N$ относительно 0.

Обозначим $\mathscr{A}^{(0)}=\bigcup_{N \neq \infty} \mathscr{A}_{N}^{(0)}$ множество всех конечных графов с корнем 0.

Графы с локальной структурой. Простым обобщением спинграф̆а является понятие графа с локальной структурой. Этого понятия достаточно для описания произвольных многомерных объектов.

ОПрЕДЕЛЕНИЕ 1. Пусть заданы функция $s(\gamma)$ на $\mathscr{A}_{d}^{(0)}$ и функция $t(\gamma)$ на множестве $\mathscr{A}(d)$ спинграфов диаметра $d$ со значениями в некотором множестве $S$. Суперлокальная структура (радиуса $d$ ) на графе $G$ задается набором значений $s\left(O_{d}(v)\right), v \in V(G)$. Локальная структура (диаметра $d$ ) на графе $G$ задается набором значений $t(\gamma)$ для всевозможных регулярных подграфов $\gamma$ диаметра $d$.

\section{ПРИМЕРЫ.}

- Спинграфы соответствуют локальной (или суперлокальной) структуре с $d=0$. Многие определения и результаты для спинграфов, полученные ниже, обобщаются на $d>0$ путем соответствуюшего переписывания.

- Калибровочные поля на графах: каждой вершине и каждому ребру ставится в соответствие значение из некоторой группы $U$; в этом случае можно взять $d=1$.

- Симплициальный комплекс полностью определяется по своему одномерному остову, если каждому полному регулярному подграфу $\gamma$ диаметра 1 его одномерного остова сопоставлена 1 , когда он определяет симплекс соответствующей размерности, и 0 - в противном случае. Здесь снова можно взять $d=1$.

- Квантовые сети Р. Пенроуза [9], [10]: в конечном графе, каждая вершина которого имеет степень 3 , каждому ребру $l$ приписывается целое число $p_{l}=2 s_{l}$, где полуцелые $s_{l}$ интерпретируются как степени неприводимых представлений группы $S U(2)$. При этом в каждой вершине должно соблюдаться условие для спинов инцидентных ей ребер: сумма трех $p_{i}$ четна и любое $p_{i}$ не превосходит суммы двух других. Тогда для тензорного произведения трех представлений в каждой вершине сушествует единственный (с точностью до множителя) инвариантньй элемент, которьй и ставится в соответствие вершине.

- Есть, однако, структуры на графах, которые не могут быть охарактеризованы как графы с локальной структурой. Примером могут служить деревья, графы Кәли груп и др. Мы увидим в дальнейшем, однако, что возможна гиббсовская (в некотором смысле тоже локальная) характеризация таких графов.

$\sigma$-алгебра и свободная мера. Пусть $\mathscr{A}$ - произвольное множество конечных спинграфов $\alpha=(G, s)$. Пусть $\mathscr{G}=\mathscr{G}(\mathscr{A})$ - множество всех графов $G$ таких, что сушествует функция $s$ с $\alpha=(G, s) \in \mathscr{A}$. Всегда предполагается, что если $\alpha=$ $(G, s) \in \mathscr{A}$, то для любой $s^{\prime}$ все $\left(G, s^{\prime}\right)$ также принадлежат $\mathscr{A}$. 
Тогда $\mathscr{A}$ - топологическое пространство, являющееся дискретным (конечным или счетным) объединением топологических пространств $T_{G}=S^{V(G)}, G \in \mathscr{G}(\mathscr{A})$. Борелевская $\sigma$-алгебра на $\mathscr{A}$ порождается цилиндрическими подмножествами $A\left(G, B_{v}, v \in V(G)\right), G \in \mathscr{G}$, где $B_{v}$ - некоторые борелевские подмножества $S$. При этом $A\left(G, B_{v}, v \in V(G)\right)$ - множество всех $\alpha=(G, s)$ таких, что граф $G$ фиксирован, а функции (конфигурации) $s(v): V(G) \rightarrow S$ удовлетворяют условию: $s(v) \in B_{v}$ для всех $v \in V(G)$.

Пусть на $S$ задана неотрицательная (не обязательно вероятностная) мера $\lambda_{0}$. Назовем свободной мерой неотрицательную меру $\lambda_{\mathscr{A}}$ на $\mathscr{A}$, определяемую посредством

$$
\lambda_{\mathscr{A}}\left(A\left(G, B_{v}, v \in V(G)\right)\right)=\prod_{v \in V(G)} \lambda_{0}\left(B_{v}\right) .
$$

Потенциалы. Потенциалом (локальным) назьвается функция $\Phi: \bigcup_{N<\infty} \mathscr{A}_{N} \rightarrow$ $\mathbb{R} \cup\{+\infty\}$, т.е. функщия на множестве конечных спинграфов, инвариантная относительно изоморфизмов. Если $\Phi$ может принимать значение $\infty$, то будем говорить, что $\Phi$ имеет твердую сердшевину.

Будем говорить, что потенциал $\Phi$ имеет конечньй диаметр, если $\Phi(\alpha)=0$ для всех $\alpha$ с диаметром более некоторой константы $d$. Минимальное такое $d$ называется диаметром $\Phi$.

Для потенщиала $\Phi$ энергию спинграфа $\alpha$ можно определить как

$$
H(\alpha)=\sum_{\gamma \subset \alpha} \Phi(\gamma), \quad \alpha=(G, s),
$$

где сумма берется по всем регулярным связным подграффам $\gamma$ спинграфа $\alpha$.

Частным случаем потенциала является химический потенциал. Он задается функцией $\Phi$, равной константе $\mu_{0}$ для каждой вершины $v$, независимо от спина в этой вершине, равной константе $\mu_{1}$ для каждого ребра и равной 0 в остальных случаях. Иначе говоря, для химического потенциала

$$
H_{N}(\alpha)=\mu_{0} V(\alpha)+\mu_{1} L(\alpha)
$$

где $V(\alpha), L(\alpha)$ - число вершин и ребер в $\alpha$.

Конечные гиббсовские семейства. Пусть $\mathscr{A}$ - некоторое множество конечных спинграфов. Гиббсовским $\mathscr{A}$-семейством с потенциалом $\Phi$ назьвается вероятностная мера $\mu_{\mathscr{A}}$ на $\mathscr{A}$, определяемая следующей плотностью по свободной мере:

$$
\frac{d \mu_{\mathscr{A}}}{d \lambda_{\mathscr{A}}}(\alpha)=Z^{-1} \exp (-\beta H(\alpha)), \quad \alpha \in \mathscr{A},
$$

где $\beta \geqslant 0$ - обратная температура. При этом мы предполагаем, что $Z \neq 0$, т.е. $H(\alpha)<\infty$ хотя бы для одного $\alpha \in \mathscr{A}$. Ниже мы приведем примеры, когда $Z=0$. Мы будем предполагать также, что, когда $\mathscr{A}$ бесконечно, вьполнено следующее условие устойчивости:

$$
Z=Z(\mathscr{A})=\int_{\mathscr{A}} \exp (-\beta H(\alpha)) d \lambda_{\mathscr{A}}<\infty .
$$

Если $S$ тривиально (состоит из одного элемента), то мы получаем вероятностное распределение на графах, которое будем назьвать гиббсовским графом.

Устойчивость. Будем говорить, что потенциал $\Phi$ устойчив на $\mathscr{A}_{N}^{(0)}$, если для любого $N$ статсумма $Z_{N}<\infty$. Потенциал $\Phi \equiv 0$ очевидно неустойчив, так же как и химический потенциал с $\mu_{0}=0$. 
ЛЕмма 1. Химический потенциал неустойчив на $\mathscr{A}_{N}^{(0)}$ для всех конечных $\mu_{0}, \mu_{1}$.

ДокАЗАТЕльство. Пусть $g(n, k)$ - число графоров радиуса 1, у которых степень вершины 0 равна $n$, а число ребер между остальными вершинами равно $k$. Достаточно показать, что

$$
G_{n}=\sum_{k=0}^{N} g(n, k) \exp \left(-\mu_{1}(k+n)-\mu_{0}(n+1)\right)
$$

растет вместе с $n$, где $N=n(n-1) / 2$ - число различных пар из $n$ занумерованных вершин.

Действительно, число графов с занумерованньми $n$ вершинами и $k$ ребрами равно $C_{N}^{k}$. В то же время число способов занумеровать $n$ вершин не превьшает $n !=$ $2^{n \log _{2} n+O(n)}$. Докажем, что для любого $\mu_{1}$ сушествует достаточно малое $\delta>0$ такое, что при $k=\delta N, n \rightarrow \infty$ будет $G_{n} \rightarrow \infty$. Имеем

$$
\begin{aligned}
& g(n, k) \exp \left(-\mu_{1}(k+n)-\mu_{0}(n+1)\right) \\
& \quad \approx 2^{-N\left(\delta \log _{2} \delta+(1-\delta) \log _{2}(1-\delta)\right)+O\left(n \log _{2} n\right)} \exp \left(-\mu_{1}(k+n)-\mu_{0}(n+1)\right) \\
& \quad>\exp \left(-\mu_{1} \delta N\right) 2^{N\left(-\delta \log _{2} \delta+\delta\right)+O\left(n \log _{2} n\right)} \rightarrow \infty,
\end{aligned}
$$

если $\delta$ достаточно мало.

Неустойчивость возникает за счет большой степени вершин. Поэтому необходима регуляризация. Одна из возможностей - вводить регуляризуюшие потенциалы.

Примером регуляризующего потенциала может быть следующий. Пусть $\gamma_{2}\left(\gamma_{3}\right)-$ граф, состояший из трех вершин 1, 2, 3 и двух ребер 12, 13 (трех ребер 12, 13, 23). Потенциал $\Phi_{2}=\mu_{2} P(\alpha)$, где $P(\alpha)$ - число регулярных подграфов графа $\alpha$, изоморфных $\gamma_{2}$ или $\gamma_{3}$, обеспечивает устойчивость потенщиала $\Phi+\Phi_{2}$ для многих $\Phi$. Например, имеет место утверждение, которое мы не доказьваем.

ПРЕДЛОЖЕНИЕ 1. Если $\mu_{2}>\ln 2$, то потенииал $\Phi=\Phi_{0}+\Phi_{1}+\Phi_{2}$ устойчив для всех $\mu_{0}, \mu_{1} \geqslant 0$.

Однако наиболее интересны потенциалы, равные $\infty$ на граффах определенного вида. Например, на всех графах диаметра 1 или 2, у которых хотя бы одна из вершин имеет степень, равную $r+1$. Рассмотрение такого потенциала эквивалентно тому, что мы ограничиваемся графами, у которых каждая вершина имеет степень, не превосходящую $r$.

Другая возможность - иначе понимать локальность.

Локальность и суперлокальность.

Во многих работах по дискретной квантовой гравитации заранее предполагается, что действие происходит на гладком или кусочно-линейном многообразии заданной размерности. Но тогда локально пространство остается классическим. Более последовательной программой был бы отказ от этого предположения. Но тогда немедленно встает центральньй вопрос: что такое локальность, если нет пространства?

И здесь сразу же выясняется, что локальность в условиях отсутствия основного пространства можно понимать по-разному. Во-первых, локальным можно называть 
объект, если он определен в терминах “малых" связных подграффов. Существенно более сильное понятие локальности (суперлокальность) возникает, когда мы знаем, что подграф̆ исчерпьвает всю окрестность данной вершины. В соответствии с этим назовем суперлокальным потенциалом радиуса $r$ функцию $\Phi: \mathscr{A}_{r}^{(0)} \rightarrow \mathbb{R} \cup\{+\infty\}$, т.е. функцию на множестве конечных корневых спинграфов радиуса $r$ относительно 0 , инвариантную относительно изоморфизмов.

Энергия для суперлокального потенциала определяется как

$$
H(\alpha)=\sum_{v \in V(\alpha)} \Phi\left(O_{r}(v)\right)
$$

где сумма берется по всем вершинам спинграфа $\alpha$. В этом случае будем говорить о суперлокальных гиббсовских семействах, а определенные вьше гиббсовские семейства будем назьвать локальными.

Суперлокальньй потенциал может быть сведен к локальному, но только с помощью дополнительного предельного перехода, см. ниже. Можно сказать, что если мы хотим описьвать мир локально (но без априорных ограничений на локальную структуру граффа), то только с перенормировками.

Для построения примеров полезно ввести следуюший класс графов. Рассмотрим набор "малых" графов $G_{1}, \ldots, G_{k}$ с корнем 0 , имеющих радиус $r$ относительно 0 . Будем говорить, что граф $G$ порожден образуюшими $G_{1}, \ldots, G_{k}$, если каждая вершина $G$ имеет окрестность (радиуса $r$ ), изоморфную одному из $G_{1}, \ldots, G_{k}$. Обозначим $\mathscr{G}\left(G_{1}, \ldots, G_{k}\right)$ - множество всех таких графов. Введем суперлокальный потенциал $\Phi=\Phi_{G_{1}, \ldots, G_{k}}$ радиуса $r$, положив $\Phi(\Gamma)=0$, если корневой подграф $\Gamma$ изоморфен одному из $G_{i}$, и $\Phi(\Gamma)=\infty$ в остальных случаях. Очевидно, что любое гиббсовское семейство с потенциалом $\Phi$ имеет носитель на $\mathscr{G}\left(G_{1}, \ldots, G_{k}\right)$.

Пример $Z_{N}=0$. Даже конечные гиббсовские семейства с потенциалом $\Phi=$ $\Phi_{G_{1}, \ldots, G_{k}}$ не всегда сушествуют. В качестве простейшего примера возьмем случай $k=1, r=1$, когда $G_{1}$ - конечньй полньй граф $g_{m}$ радиуса 1 с $m+1$ вершиной $0,1,2,3, \ldots, m$ и корнем 0 . Тогда он “самопорождаюший” и $\mathscr{G}\left(G_{1}\right)$ состоит из одного элемента - самого $g_{m}$.

Другой пример. Введем граф̆ $g_{m, 0} \mathrm{c} m+1$ вершиной $0,1,2,3, \ldots, m$ и $m$ ребрами $01,02, \ldots, 0 m$ и граф $g_{m, m}$ с $m+1$ вершиной $0,1,2,3, \ldots, m$ и $2 m$ ребрами $01,02, \ldots$, $0 m, 12,23, \ldots, m 1$. Можно видеть, что пентагон $G_{1}=g_{5,5}$ не может породить счетного графа.

Перенормировка. Суперлокальньй потенциал может быть выражен с помощюю локальных следуюшим образом. Мы поясним это для случая $k=1$, пусть радиус $G_{1}$ равен $r$. Мы построим конечное гиббсовское семейство с носителем на $\mathscr{G}\left(G_{1}\right)$, не используя суперлокальных потенциалов. Пусть $F\left(G_{1}\right)$ - множество всех граффов, изоморфных какому-либо собственному регулярному подграфу $\gamma$ графа $G_{1}$ такому, что радиус граф̆а $\gamma$ относительно некоторой вершины $v \in V(\gamma)$ равен $r$. Пусть $F(\Gamma, \gamma)-$ множество всех собственных регулярных подграфов графиа $\Gamma$, изоморфных $\gamma$.

Введем урезанньй локальный потенщиал $\Phi_{R}$, полагая

- $\Phi_{R}(\gamma)=\infty$ для графов $\gamma$, имеюших радиус $r$ относительно некоторой своей вершины и таких, что $\gamma \neq G_{1}$ и $\gamma \notin F\left(G_{1}\right)$; 
- $\Phi_{R}(\gamma)=0$ для графов, не имеющих радиуса $r$ относительно никакой из своих вершин;

- $\Phi_{R}(\gamma)=R, R>0$, для любого графа $\gamma \in F\left(G_{1}\right)$;

- $\Phi_{R}\left(G_{1}\right)=0$.

Предел при $R \rightarrow \infty$ гиббсовского семейства $\mu_{\mathscr{A}}(R)$ с потенциалом $\Phi_{R}$ может не существовать, однако имеет место

Теорема 1. Предположим, что $\mathscr{G}\left(G_{1}\right) \cap \mathscr{A}_{N} \neq \varnothing$. Тогда существуют такое $k$ и такие локальные потенциаль $\phi_{R}^{(j)}, j=1,2, \ldots, k$, что предел при $R \rightarrow \infty$ гиббсовского семейства на $\mathscr{A}_{N}$ с перенормированным потенциалом

$$
\Phi_{R}^{\mathrm{ren}}=\Phi_{R}+\sum_{j=1}^{k} \phi_{R}^{(j)}
$$

существует и имеет носитель на $\mathscr{G}\left(G_{1}\right) \cap \mathscr{A}_{N}$.

ДокАЗАТЕльство. Введем потенциалы (контрчлены) $\phi_{R}^{(j)}$, равные нулю везде, кроме следующих случаев:

- если Г изоморфен $G_{1}$, то

$$
\phi_{R}^{(1)}(\Gamma)=\sum_{\gamma \in F\left(G_{1}\right)} \phi_{R, \gamma}^{(1)}(\Gamma), \quad \phi_{R, \gamma}^{(1)}(\Gamma)=-R \sum_{\Gamma \in F\left(G_{1}, \gamma\right)} 1 ;
$$

- для любого $j \geqslant 2$ если Г является объединением $j$ попарно различных подграффов $\Gamma_{i}, i=1, \ldots, j$, причем каждьй $\Gamma_{i}$ изоморфен $G_{1}$, то

$$
\phi_{R}^{(j)}(\Gamma)=\sum_{\gamma \in F\left(G_{1}\right)} \phi_{R, \gamma}^{(j)}(\Gamma), \quad \phi_{R, \gamma}^{(j)}(\Gamma)=(-1)^{j} R \sum_{\Gamma \in F\left(\Gamma_{1}, \gamma\right) \cap \cdots \cap F\left(\Gamma_{j}, \gamma\right)} 1 .
$$

Заметим, что при таком определении $\phi_{R}^{(j)}=0$ начиная с некоторого $j$. Рассмотрим некоторую вершину $v$ графра. Если ее окрестность $O_{r}(v)$ не принадлежит $F\left(G_{1}\right)$, то потенциал $\Phi_{R}$ равен $\infty$, и, значит, это невозможно. Пусть теперь окрестность $O_{r}(v)$ не изоморфна $G_{1}$ и принадлежит $F\left(G_{1}\right)$. Тогда потенциал $\Phi_{R}$ на ней равен $R$, а контрчлены равны 0 , откуда и $\Phi_{R}^{\mathrm{ren}}$ равен $R$. Рассмотрим теперь некоторый подграф $\gamma \in F\left(G_{1}\right)$, которьй принадлежит ровно $m$ подграфам $\Gamma_{i}$, изоморфным $G_{1}$. Тогда по формуле включения-исключения для любого $\gamma$

$$
\Phi_{R}(\gamma)+\sum_{j=1}^{m} \phi_{R, \gamma}^{(j)}(g)=0
$$

Поэтому энергия графов из $\mathscr{G}\left(G_{1}\right) \cap \mathscr{A}_{N}$ равна 0, а для всех остальных она не менее $R$, что и дает результат.

Заметим, что $\phi_{R}^{(j)}(\Gamma)$ аналогичны контрчленам обычной теории перенормировок. При $k>1$ возникает более сложная комбинаторика. Возможно, для некоторых моделей с вещественным спином сушествует связь этих контрчленов с контрчленами стандартной теории перенормировок. 


\section{2. Предельные гиббсовские семейства.}

Предельные гиббсовские семейства. Введем топологию на множестве $\mathscr{A}_{\infty}^{(0)}$ счетных спинграфов с корнем 0, определив базис открытых множеств на нем. Пусть $C_{N}$ - произвольное открытое подмножество множества $\mathscr{A}_{N}^{(0)}$ всех спинграфов с радиусом $N$ относительно начала координат. Тогда открытые множества базиса состоят из всех счетных спинграфов таких, что (для некоторого $N$ и некоторого $C_{N}$ ) их $N$-окрестность начала координат принадлежит $C_{N}$. В качестве $\sigma$-алгебры в $\mathscr{A}_{\infty}^{(0)}$ возьмем борелевскую $\sigma$-алгебру, порожденную этими открытыми множествами.

Для любого спинграфа $\alpha$ с корнем 0 назовем $\gamma(\alpha, 0 ; N, N+d) \quad N$-разрезом ширины $d, \gamma(\alpha, 0 ; 0, N)-N$-внутренней, $\gamma(\alpha, 0 ; N, \infty)-N$-внешней частями соответственно. Заметим, что $N$-разрез и $N$-внешняя часть могут оказаться несвязными. Для данных $N, d$ и некоторого конечного графа $\gamma$ (не обязательно связного) пусть $\mathscr{A}_{N+d}^{(0)}(\gamma, d) \subset$ $\mathscr{A}_{N+d}^{(0)}$ - множество всех конечных связных спинграфов $\alpha$ с радиусом $N+d$ относительно начала координат, имеюших $\gamma$ в качестве $N$-разреза ширины $d$.

Пусть задан локальный потенщиал $\Phi$ диаметра $d+1$.

ОПРЕДЕЛЕНИЕ 2 . Назовем меру $\mu$ на $\mathscr{A}_{\infty}^{(0)}$ гиббсовским семейством с локальньм потенциалом $\Phi$, если для любых $N, \gamma, \Gamma$ условное распределение на множестве спинграфов $\alpha \in \mathscr{A}_{\infty}^{(0)}$, имеюших $N$-внешнюю часть $\Gamma$ и $N$-разрез $\gamma$ ширины $d$ (это множество можно отождествить с $\left.\mathscr{A}_{N+d}^{(0)}(\gamma, d)\right)$, совпадает п.н. с гиббсовским семейством с потенциалом $\Phi$ на $\mathscr{A}_{N+d}^{(0)}(\gamma, d)$.

В частности, это условное распределение зависит только от $\gamma$, но не от всей $N$-внешней части $Г$.

Граничные условия. Для нахождения чистых фаз, как и в обычной теории, полезно понятие гиббсовского семейства с заданными граничными условиями. Граничные условия ( $d$-граничные условия) задаются последовательностью $\nu_{N, d}(\gamma)$ вероятностных мер на множестве конечных (не обязательно связных) спинграффов $\gamma$, интуитивно - на $N$-разрезах ширины $d$. Гиббсовское семейство на $\mathscr{A}_{N}^{(0)}$ с граничньми условиями $\nu_{N+1, d}(\gamma)$ определяется как

$$
\begin{gathered}
\mu_{N}(\alpha)=Z^{-1}\left(N, \nu_{N+1, d}\right) \sum_{\xi \in \mathscr{A}_{N+d+1}^{(0)}(\gamma, d ; \alpha)} \int \exp (-\beta H(\xi)) d \nu_{N+1}(\gamma, d), \quad \alpha \in \mathscr{A}_{N}^{(0)}, \\
Z\left(N, \nu_{N+1, d}\right)=\int_{\mathscr{A}_{N}^{(0)}}\left[\sum_{\xi \in \mathscr{A}_{N+d+1}^{(0)}(\gamma, d ; \alpha)} \int \exp (-\beta H(\xi)) d \nu_{N+1}(\gamma, d)\right] d \lambda_{\mathscr{A}_{N}^{(0)}},
\end{gathered}
$$

где $\mathscr{A}_{N+d+1}^{(0)}(\gamma, d ; \alpha)$ - множество всех спинграфов из $\mathscr{A}_{N+d+1}^{(0)}$ с $(N+1)$-разрезом $\gamma$ ширины $d$ и $N$-внутренней частью $\alpha$. Заметим, что $\mathscr{A}_{N+d+1}^{(0)}(\gamma, d ; \alpha)$ конечно для любых $\alpha$ и $\gamma$.

Компактность. Гиббсовские семейства на множестве $\mathscr{A}_{\infty}^{(0)}$ связных счетных спинграфов с корнем 0 могут быть получены как слабые пределы гиббсовских семейств на $\mathscr{A}_{N}^{(0)}$. В принципе возможны три причины, почему предельное гиббсовское семейство может не сушествовать: несуществование конечных гиббсовских семейств с этим 
потенциалом (см. примеры вьше), некомпактность $S$, а также - распределение конечных гиббсовских семейств может концентрироваться на графах с вершинами большой степени. Предположения следующего утверждения соответствуют этому списку, однако компактность, конечно, может иметь место при более общих условиях.

Пусть $\mathscr{A}_{\infty}^{(0)}(r) \subset \mathscr{A}_{\infty}^{(0)}$ - множество счетных спинграфов с вершинами степени, не большей $r$.

ПРЕДЛОЖЕНИЕ 2. Пусть $S$ компактно. Пусть $\Phi$ имеет конечный диаметр, и пусть $\Phi(\gamma)=\infty$, если хотя бы одна вериина $\gamma$ имеет степень $r+1$. Пусть для всех $N$ существует гиббсовское семейство с потенциалом $\Phi$ на $\mathscr{A}_{N}^{(0)}$. Тогда существует гиббсовское семейство с потенииалом $\Phi$, сосредоточенное на $\mathscr{A}_{\infty}^{(0)}(r)$.

ДоказАТельство. Рассмотрим гиббсовские семейства $\mu_{N}$ на $\mathscr{A}_{N}^{(0)}$, фактически на $\mathscr{A}_{N}^{(0)}(r)$. Пусть $\widetilde{\mu}_{N}$ - произвольная вероятностная мера на $\mathscr{A}_{\infty}^{(0)}(r)$ такая, что ее фактор-мерана $\mathscr{A}_{N}^{(0)}(r)$ совпадает с $\mu_{N}$. Тогда последовательность мер $\widetilde{\mu}_{N}$ на $\mathscr{A}_{\infty}^{(0)}(r)$ компактна, что может быть доказано стандартной диагональной процедурой нумерации всех $N$-окрестностей. Ясно, что всякая предельная точка последовательности $\widetilde{\mu}_{N}$ - гиббсовское семейство с потенциалом $\Phi$.

Гиббсовские семейства и гиббсовские поля. Из следуюшего простого результата будет ясно, почему мы используем термин “гиббсовское семейство". Пусть $\mu$ - гиббсовское семейство с потенциалом $\Phi$. Предположим, что выполнены условия предыдущего предложения. Рассмотрим измеримое разбиение множества $\mathscr{A}_{\infty}^{(0)}$ : любой элемент $S_{G}$ этого разбиения определяется фиксированньм графом $G$ (без спинов) и состоит из всех конфигураций $s_{G}$ на $G$.

ПРЕДЛОЖЕНИЕ 3. Для данного графа $G$ условная мера на множестве конфигураций $S_{G}=\left\{s_{G}\right\}$ является п.н. гиббсовской мерой с тем жсе потенциалом $\Phi$.

Заметим, что “тем же” имеет следуюший очевидный смысл: если $\Lambda_{N}-N$-окрестность нуля в $G$ и $s_{\Lambda_{N}}$ - конфигурация на $\Lambda_{N}$, то $H\left(s_{\Lambda_{N}}\right)=\sum \Phi(\gamma)$, где сумма берется по всем подграффам спинграфа $\left(\Lambda_{N}, s_{\Lambda_{N}}\right)$. Таким образом, любое гиббсовское семейство является вьпуклой комбинацией (весьма специальной природы) гиббсовских мер (полей) на фиксированных графах, с тем же потенциалом. Эта вьпуклая комбинация определяется мерой $\nu=\nu(\mu)$ на фактор-пространстве $\mathscr{G}_{\infty}^{(0)}=\mathscr{A}_{\infty}^{(0)} /\left\{S_{G}\right\}$, индуцированной мерой $\mu$, соответствующей самому гиббсовскому семейству.

Следующая лемма (имеющая хорошо известный и полезньй аналог для обычных гиббсовских полей) сводит доказательство утверждения к соответствуюшему результату для гиббсовских семейств на конечных графах. Результат же для конечных графов - прямое вычисление, которое мы опускаем.

Лемма 2. Пусть $\mu$ - гиббсовское семейство на $\mathscr{A}_{\infty}^{(0)}$ с потенциалом $\Phi$. Пусть $\nu_{N+1, d}(\gamma)$ - мера на $N$-разрезах ширины $d$, индуцированная $\mu$. Тогда $\mu$ является слабым пределом гиббсовских семейств на $\mathscr{A}_{N}^{(0)}$ с потенциалом $\Phi$ и граничными условиями $\left\{\nu_{N+1, d}(\gamma)\right\}$. 
2.3. Эмпирические распределения. Вероятностные меры на $\mathscr{A}_{\infty}^{(0)}$ можно строить стандартным образом, используя колмогоровский подход с согласованными распределениями на цилиндрических подмножествах. Однако этот подход не работает, если мы хотим построить вероятностные меры на множестве $\mathscr{A}_{\infty}$ классов эквивалентности счетных связных спинграфов. Проблема в том, что нет единой естественной нумерации вершин и поэтому непонятно, как вводить конечномерные распределения. Можно сказать, что проблема - в отсутствии координатной системы, точки отсчета. Поэтому колмогоровский метод не работает. Однако, некий аналог конечномерных распределений существует. Мы назьваем полученную систему чисел эмпирическим распределением на $\mathscr{A}_{\infty}$.

Пусть $S$ конечно или счетно. Рассмотрим систему чисел

$$
\pi=\left\{p(\Gamma), \Gamma \in \mathscr{A}^{(0)}\right\}, \quad 0 \leqslant p(\Gamma) \leqslant 1,
$$

т.е. $Г$ - конечные спинграффы с корнем 0. Введем свойство согласованности этих чисел для любого $k=0,1,2, \ldots$ и любого фиксированного спинграфа $\Gamma_{k}$ радиуса $k$ (относительно 0)

$$
\sum_{\Gamma_{k+1}} p\left(\Gamma_{k+1}\right)=p\left(\Gamma_{k}\right), \quad k=0,1,2, \ldots
$$

где сумма по всем спинграфам $\Gamma_{k+1}$ радиуса $k+1$ таким, что $O_{k}(0)$ в $\Gamma_{k+1}$ изоморфина $\Gamma_{k}$. Напомним, что в последней формуле предполагается, что сумма берется по всем классам эквивалентности спинграфов.

Постулируем также условие нормировки

$$
\sum p\left(\Gamma_{0}\right)=1
$$

где $\Gamma_{0}$ - вершина 0 с произвольным спином на ней.

ОПРЕДЕЛЕНИЕ 3 . Произвольная такая система $\pi$ назьвается эмпирическим распределением.

Можно переписать условие согласования в терминах условных вероятностей:

$$
\sum_{\Gamma_{k+1}} p\left(\Gamma_{k+1} \mid \Gamma_{k}\right)=1, \quad k=0,1, \ldots,
$$

где суммирование, как и выше, и

$$
p\left(\Gamma_{k+1} \mid \Gamma_{k}\right)=\frac{p\left(\Gamma_{k+1}\right)}{p\left(\Gamma_{k}\right)} .
$$

Таким образом, мы рассматриваем $\mathscr{A}^{(0)}$ как дерево, вершины которого - спинграфы, а ребро между $\Gamma_{k+1}$ и $\Gamma_{k}$ существует тогда и только тогда, когда $\Gamma_{k}$ изоморфен $k$-окрестности 0 в $\Gamma_{k+1}$.

Очевидно, система $\pi$ определяет вероятностную меру на $\mathscr{A}_{\infty}^{(0)}$. Естественность ее интерпретации как некоторой “меры” на $\mathscr{A}_{\infty}$ определяется следующими примерами.

Примеры эмпирических распределений могут быть получены с помощью следующей предельной процедуры, которую будем называть эмпирическим пределом. Пусть 
$\mu_{N}$ - вероятностная мера на $\mathscr{A}_{N}$. Для любых $N, k$ и любых спинграфов $\Gamma \in \mathscr{A}_{k}^{(0)}$, $\alpha \in \mathscr{A}_{N}$ положим

$$
p^{N}(\Gamma)=\left\langle\frac{n^{N}(\alpha, \Gamma)}{N}\right\rangle_{\mu_{N}},
$$

где $n^{N}(\alpha, \Gamma)$ - число вершин в $\alpha$, имеюших $k$-окрестности, изоморфные спинграфу Г. Положим $\pi_{N}=\left\{p^{N}(\Gamma)\right\}$. В качестве мер $\mu_{N}$ можно брать гиббсовские семейства на $\mathscr{A}_{N}$ с фиксированным потенциалом. Сама система $\pi_{N}$ не является эмпирическим распределением. Однако имеет место

ЛЕмма 3. Пусть $S$ конечно, и пусть для любого $D$

$$
\mu_{N}\left(\min _{\alpha \in \mathscr{A}_{N}} D(\alpha) \leqslant D\right) \rightarrow 0
$$

при $N \rightarrow \infty$, где $D(\alpha)$ - диаметр $\alpha$. Тогда любая слабая предельная точка системы $\pi_{N}$ является эмпирическим распределением.

ДокАЗАТЕльство. Заметим, что вероятность $\mu_{N}$ того, что для всех вершин $v$ случайного граффа имеет место $R_{v}(\alpha)>D$, стремится к 1 при $N \rightarrow \infty$. Поэтому для любых $n_{0}, \delta>0$ сушествует $N_{0}=N_{0}\left(n_{0}, \delta\right)$ такое, что для любого $N>N_{0}$ числа $p_{N}(\Gamma)$ удовлетворяют условиям согласования для всех $\Gamma_{k}$ с $k<n_{0}$ с точностью до $\delta$. Из конечности $S$ следует компактность, т.е. сушествование хотя бы одного предельного эмпирического семейства.

ЗАмечАниЕ 1. Возникает вопрос, как характеризовать эмпирические распределения, которые могут быть получены приведенным выше способом, и, в частности, какие эмпирические распределения могут быть получены из самих себя, т.е. как пределы чисел

$$
p^{N}(\Gamma)=\frac{\left\langle n^{N}\left(\Gamma_{N}, \Gamma\right)\right\rangle_{\pi_{N}}}{\left\langle V\left(\Gamma_{N}\right)\right\rangle_{\pi_{N}}}
$$

где $V\left(\Gamma_{N}\right)$ - число вершин в $\Gamma_{N}, \pi_{N}$ - ограничение меры $\pi$ на $\mathscr{A}_{N}^{(0)}$.

ЗАмечАниЕ 2. Заметим, что эмпирическая мера в очевидном смысле автоматически "однородна", одинакова в любой вершине. Причиной этого служит то, что вершины не занумерованы, или граф не вложен ни в какое пространство. Заметим, однако, что на $\mathscr{A}_{\infty}^{(0)}$ неоднородность легко вводится - можно взять $\Phi$ зависяшим от расстояния от 0 .

ЗАмЕчАНИЕ 3. Возможен подход к эмпирическим распределениям, основанный не на суперлокальности, а на локальности, когда мы “не знаем” всей окрестности вершины. Пусть, например, заданы два графа $\gamma \subset \Gamma$ таких, что в $Г$ нет другого подграфа, изоморфного $\gamma$. Тогда можно искать пределы отношений (условные эмпирические вероятности)

$$
\left\langle\frac{n^{N}(\alpha, \Gamma)}{n^{N}(\alpha, \gamma)}\right\rangle_{\mu_{N}}
$$


2.3.1. Эмпирические гиббсовские семейства. Покажем, что иногда предельные гиббсовские семейства на $\mathscr{A}_{\infty}^{(0)}$ можно получать пределом по числу вершин, а не по радиусу. Пусть $\mathscr{B}_{N, p}^{(0)}$ - множество спинграфов с $N$ вершинами, причем каждая имеет степень не более $p$, и корнем 0 . Рассмотрим конечное гиббсовское семейство $\mu_{N}$ на $\mathscr{B}_{N, p}^{(0)}$ с локальным потенциалом $\Phi$.

ЛЕмма 4. Любая слабая предельная точка последовательности $\mu_{N}$ является предельным гиббсовским семейством на $\mathscr{A}_{\infty}^{(0)}$ с потенииалом $\Phi$.

ДокАЗАТЕльство. Фиксируем $n, d$ и некоторый $\alpha \in \mathscr{A}_{n+d+1, p}^{(0)}$ с $n$-разрезом $\gamma$ ширины $d+1$. Тогда для всех достаточно больших $N$ сушествует $\beta \in \mathscr{B}_{N, p}^{(0)}$ такой, что $O_{n+d+1}(\beta, 0)$ изоморфна $\alpha$. Для любого такого $\beta$ условное распределение $O_{n}(\alpha, 0)$ при условии $\gamma$ является гиббсовским с потенциалом $\Phi$.

Эмпирическое распределение $\pi$ на $\mathscr{A}_{\infty}$ назьвается гиббсовским, если оно получено как эмпирический предел конечных гиббсовских семейств $\pi_{N}$ на $\mathscr{A}_{N}$ с потенциалом $\Phi$.

Чтобы оправдать это определение, мы покажем, что при некоторых условиях эмпирическое распределение $\pi$ на $\mathscr{A}_{\infty}$ является гиббсовским семейством как мера на $\mathscr{A}_{\infty}^{(0)}$. Предположим, что эмпирическое распределение $\pi$ на $\mathscr{A}_{\infty}$ является гиббсовским с потенциалом $\Phi$, равным $\infty$, если степень хотя бы одной вершины более $p$, и что вероятность $\pi_{N}$ того, что группа автоморфизмов графа $\alpha \in \mathscr{A}_{N}$ тривиальна, стремится к 1 при $N \rightarrow \infty$. Тогда $\pi$ является гиббсовским семейством как мера на $\mathscr{A}_{\infty}^{(0)}$. Действительно, если графф имеет тривиальную группу автоморфизмов, то можно поместить корень в любую его вершину, получая каждый раз разные графы. Тогда мы имеем гиббсовские семейства на $\mathscr{B}_{N, p}^{(0)}$, и по предыдущей лемме получаем предельное гиббсовское семейство на $\mathscr{A}_{\infty}^{(0)}$.

2.3.2. Эмпирические распределения в планарной гравитации. Следует подчеркнуть, что вопрос о существовании предельных корреляционных функций, который принято игнорировать в физике, далеко не тривиален. Помимо математической естественности есть еще один аспект этого вопроса, представляющий интерес и для физики. Дело в том, что при принятом в дискретной квантовой гравитации определении распределения с помошью большого канонического ансамбля термодинамический предельный переход имеет смысл только в некоторой критической точке, определяющей гранищу сходимости ряда, которая, более того, сильно зависит от геометрических деталей даже при фиксированной топологии многообразия. При суммировании по топологиям даже в размерности 2 соответствуюший ряд расходится для всех значений параметров из-за факториальной асимптотики энтропии. В каноническом ансамбле, для нашего определения гиббсовских распределений, таких трудностей не существует.

Здесь мы приведем популярные примеры эмпирических гиббсовских распределений. В физике двумерная планарная квантовая гравитация (или струна в размерности 0) определяется большой статистической суммой

$$
P(T)=Z^{-1} \exp (-\mu F(T)), \quad Z=\sum_{T \in \mathscr{A}} \exp (-\mu F(T))=\sum_{N} C(N) \exp (-\mu N),
$$


где первая сумма берется по всем триангулящиям $T$ сферы с $N$ треугольниками, принадлежашим некоторому классу $\mathscr{A}$ триангуляций. Точное определение класса $\mathscr{A}$ не играет существенной роли, см. [11], но ведет, однако, к разным эмпирическим распределениям для разных $\mathscr{A} . C(N)=C_{\mathscr{A}}(N)$ назьвается энтропией и равно числу таких триангулящий. Известно, что

$$
C(N) \sim a N^{-\frac{7}{2}} c^{N}
$$

При желании $N$ можно считать числом вершин двойственного граффа, которьй является 3-регулярным графом, вложенным в двумерную сферу. Большой ансамбль рассматривается при $\mu \rightarrow \mu_{\mathrm{cr}}=-\log c$. В каноническом ансамбле нет необходимости вводить параметр $\mu$.

Рассмотрим триангуляии с выделенной вершиной 0 и фиксированной окрестностью $O_{d}(0)=\Gamma_{d}$ радиуса $d$. Пусть $p^{N}\left(\Gamma_{d}\right)$ - ее вероятность в нашем каноническом ансамбле.

Теорема 2. Существует предел $\lim p^{N}\left(\Gamma_{d}\right)=\pi\left(\Gamma_{d}\right)$, который определяет әмпирическое распределение.

Мы приведем доказательство, используюшее явные формулы. Пусть $\Gamma_{d}$ имеет $u$ треугольников и $k$ граничных ребер (т.е. ребер с обеими вершинами на $d$-срезе). Пусть $r(d, k, u)$ - число таких окрестностей. Внешняя часть является триангуляцией диска с $N-u$ треугольниками и с $k$ граничными ребрами. Число $D(N-u, k)$ таких триангуляций (это следует из результатов Татта, см. [11]) имеет следующую асимптотику при $N \rightarrow \infty$ :

$$
D(N-u, k) \sim \phi(k) N^{-\frac{5}{2}} c^{N-u} .
$$

Поэтому вероятность $\Gamma_{d}$ (в вершине 0) равна

$$
P^{N}\left(\Gamma_{d}\right) \sim \frac{\phi(k) N^{-\frac{5}{2}} c^{N-u}}{\sum_{u, k} r(d, k, u) \phi(k) N^{-\frac{5}{2}} c^{N-u}}=\frac{\phi(k) c^{-u}}{\sum_{u, k} r(d, k, u) \phi(k) c^{-u}} .
$$

Функции $\phi, r$ и константа $c$ известны в явном виде, но нам это не важно. Из того, что почти все графы имеют тривиальную групу автоморфизмов, следует, что эмпирический предел такой же.

Заметим, что если вместо сферы мы взяли бы поверхность рода $\rho$, то получили бы другое эмпирическое распределение, зависящее от $\rho$ и $\mathscr{A}$.

Является ли это эмпирическое распределение (планарное!) гиббсовским эмпирическим семейством? Можно выделить двумерные триангулящии с помошью потенщиала $\Phi$ такого, что $\Phi\left(O_{1}(v)\right)=\infty$, если $O_{1}(v) \neq g_{k, k}, k \geqslant 2$. Однако одновременно мы получим триангуляции любой двумерной поверхности. Поэтому остается два способа. Первый - получить триангуляции сферы как чистую фазу некоторого гиббсовского семейства. Подобный пример мы строим ниже. Второй способ: рассматривать нелокальные потенщиалы (бесконечного радиуса). Последняя характеризация хорошо известна в теории графов. Положим $\Phi\left(K_{5}\right)=\Phi\left(K_{3,3}\right)=\infty$, если $K_{5}$ является (нерегулярным) подграфом, топологически изоморфным полному графу с 5 вершинами, а $K_{3,3}$ - (нерегулярньй) подграф, топологически изоморфньй графу с 6 вершинами 
$1,2,3,4,5,6$ и всеми ребрами вида $(i, j), i=1,2,3, j=4,5,6$ (по теореме Понтрягина-Куратовского это выделяет плоские подграфы). Положим $\Phi=0$ в остальных случаях. Пока планарность следует рассматривать как априорное условие глобального характера. Заметим, однако, что если нет ограничения на род поверхности, то мы очевидно имеем гиббсовское распределение.

\section{3. Энтропия}

Знаменитая теорема ван Хова в статистической физике утверждает, что статистическая сумма растет экспоненщиально с объемом. В квантовой гравитации этот результат, касательно числа триангуляций (энтропии), был предметом многочисленных исследований, см. [12]. При этом рассматривался только случай, когда топология многообразия фиксирована или подчинена существенным ограничениям (см. [13]). Однако нетрудно видеть, что статсумма растет быстрее чем экспоненциально даже в двумерном случае, если не фиксировать род поверхности. Поэтому принятьй в физических работах большой канонический ансамбль не сушествует, если имеется сверхэкспоненщиальный рост канонической статсуммы. Двойной скейлинг-предел является (нестрогой) попыткой преодолеть эту трудность.

С другой стороны, интересен вопрос, в каких пределах вообще может меняться энтропия. Ниже мы встретимся с интересным случаем степенного роста энтропии, где будет фазовый переход. В то же время общее число $p$-регулярных графов имеет рост порядка $N^{\frac{p N}{2}}$. Заметим, что добавление спина в гиббсовский граф может влиять только на те топологии, которые типичны (т.е. отличаются от максимальной энтропии разве что экспоненциальным множителем). Поэтому надо знать грубую, например логарифмическую, асимптотику энтропии.

3.1. Произвольный род в размерности 2. Напомним, что асимптотика числа триангулящий поверхности фиксированного рода $g$ имеет вид

$$
C(N, g) \sim f(g) N^{a g+b} c^{N}, \quad a=\frac{5}{2}, \quad b=-\frac{5}{2}-1,
$$

причем $c$ не зависит от $g$. Мне неизвестно строгое формальное доказательство этого результата, но он аналогичен результатам в [14] для других классов карт, полученным прямьм, но громоздким, развитием метода Татта. Заметим, что сведение задачи к матричной модели хотя и приводит к такому же результату, но не дает строгого доказательства. Намного сложнее случай, когда род не фиксирован. Мы приведем здесь некоторые первые результаты на этот счет, см. также [15].

Приведем необходимые определения из [16]. Картой назьвается тройка $(S, G, \phi)$, где $S$ - замкнутое ориентированное связное двумерное многообразие, $G$ - связньй граф, $\phi$-гомеоморфное вложение $G$ в $S$ такое, что дополнение к $\phi G$ является объединением связных открытых областей, каждая из которых гомеоморфна диску. Корневая карта - карта с выделенным отростком (т.е. конец одного из ребер) одной из вершин $G$. Две корневые карты (комбинаторно) эквивалентны, если сушествует сохраняющий ориентацию гомеоморфизм $S$, переводящий в себя множество вершин, множество ребер и корень. Сушествует эквивалентное чисто комбинаторное определение карт как упорядоченного графа. Упорядоченньм (или ленточным) графом называется граф с заданным циклическим порядком отростков для каждой вершины. Это определение основано на следуюшей теореме. 
ТЕОРема 3. Для каждого связного упорядоченного графа существует единственная с точностью до әквивалентности карта, у которой порядок отростков соответствует движению по часовой стрелке.

Пусть $E$ - множество ребер, тогда $2 E=E \times\{-1,1\}$ можно отождествить с множеством отростков, вершины определяются разбиением множества $2 E$. Разбиение вместе с циклическим порядком внутри каждого его элемента задаются подстановкой $P$ на $2 E$. Другая подстановка $(-)$, переставляет отростки одного ребра, т.е. определяет разбиение множества $2 E$ на ребра. Диски (грани) определяются последовательностью отростков $e,(-) e, P(-) e,(-) P(-) e, \ldots$, соответствуюшей обходу границы диска. Таким образом, число граней равно числу циклов перестановки $P(-)$. Это объяснение позволяет дать следующее определение.

ОПРЕДЕЛЕНИЕ 4. Комбинаторная карта есть тройка $(2 E, P,(-))$, состоящая из множества $2 E$ с четным числом элементов и двух его перестановок таких, что группа, порожденная ими, транзитивна на этом множестве (это соответствует связности графа). Требуется также, чтобы перестановка (-) состояла из непересекающихся циклов длины 2. Корень - выделенньй элемент множества $2 E$.

Пусть $F_{b, p}(g)$ - число корневых карт с $p+1$ вершиной и $b+p$ ребрами на поверхности рода $g$. Положим $F_{b, p}=\sum_{g} F_{b, p}(g)$. Основной наш результат таков.

ТЕОРема 4. Пусть $p(b)$ - неубывающая последовательность иелых чисел такая, что $p(b) / b \rightarrow \alpha$ для некоторой константы $0<\alpha<\infty$. Тогда

$$
b^{-1} \ln \frac{F_{b, p(b)}}{b !} \rightarrow c=c(\alpha), \quad 0<c(\alpha)<\infty .
$$

В работе [16] приводятся следуюшие рекуррентные соотношения для чисел $F_{b, p}$ :

$$
F_{b, p}=\sum_{j=0}^{p-1} \sum_{k=0}^{b} F_{k, j} F_{b-k, p-j-1}+(2(b+p)-1) F_{b-1, p}, \quad b, p \geqslant 0
$$

кроме случая $b=p=0$. При этом

$$
F_{0,0}=1, \quad F_{-1, p}=F_{b,-1}=0
$$

Отдельно надо проверить случаи $b=0$ или $p=0$. Случай $b=p=0$ соответствует вложению одной вершины в сфреру. Случаи $p \geqslant 1, b=0$ соответствуют дереву, вложенному также в сферу, $F_{0, p}=\frac{(2 p) !}{p !(p+1) !} \cdot F_{b, 0}, b \geqslant 1$, равны $(2 b-1)(2 b-3) \cdots=\frac{(2 b) !}{b ! 2^{b}}$ - числу разбиений множества $\{1,2, \ldots, 2 b\}$ на пары.

Лемма 5. Имеют место следующие оценки:

$$
c_{1}^{b+p}<G_{b, p}=\frac{F_{b, p}}{b !}<c_{2}^{b+p}, \quad 0<c_{1}<c_{2}<\infty
$$


ДокАЗАТЕЛЬСТво. Имеем из формулы (17)

$$
G_{b, p}=\sum_{j=0}^{p-1} \sum_{k=0}^{b} \frac{k !(b-k) !}{b !} G_{k, j} G_{b-k, p-j-1}+b^{-1}(2(b+p)-1) G_{b-1, p}, \quad b, p \geqslant 1 .
$$

Оценка снизу получается из минорируюшего рекуррентного соотношения

$$
E_{b, p}>2 E_{b-1, p}, \quad b \geqslant 1, \quad p \geqslant 1, \quad E_{0, p}=G_{0, p}=\frac{(2 p) !}{p !(p+1) !} .
$$

Для оценки сверху нам понадобится некоторая техника - разложение по деревьям, которое соответствует итерированию мажорируюшего соотношения

$$
G_{b, p}=\sum_{j=0}^{p-1} \sum_{k=0}^{b} \frac{k !(b-k) !}{b !} G_{k, j} G_{b-k, p-j-1}+\left(2+\frac{2 p}{b}\right) G_{b-1, p}
$$

с теми же граничными условиями $G_{0,0}=1, G_{b, p}=0$, если либо $p=-1$, либо $b=-1$. Мы будем рассматривать пары $(T, \phi)$, где $T-$ конечное корневое дерево, $\phi$ - отображение множества вершин $V(T)$ в положительньй квадрант плоскости, обозначая $\phi(v)=(b=b(v), p=p(v))$ для $v \in V(T)$. Пусть $\Phi(b, p)$ - множество таких пар, у которых корень соответствует точке $(b, p)$. Каждому такому дереву соответствует итерация соотношения (19) вплоть до некоторой остановки. Построение новой вершины (будем говорить, что они находятся под старой вершиной) соответствует одному шагу итерации: мы либо выбираем один из членов правой части соотношения (19), либо останавливаемся для данной вершины. Из каждой вершины $v=(b, p)$ дерева могут расти два ребра, в этом случае вершина назьвается бинарной и две другие вершины этих ребер находятся в точках $(k, j)$ и $(b-k, p-j-1)$. При этом вершине $(b, p)$ приписывается число $f(v)=\frac{k !(b-k) !}{b !}$. Из вершины может расти только одно ребро, в этом случае вершина назьвается унарной, а другая вершина этого ребра находится в точке $(b-1, p)$. В этом случае вершине $v=(b, p)$ приписьвается число $f(v)=2+2 p / b$. Вершина $v=(b, p)$, из которой не растет ребер, назьвается финальной, и ей приписывается число $f(v)=G_{b, p}$. Вкладом дерева $T$ называется произведение

$$
f(T)=\prod_{v \in V(T)} f(v)
$$

Будем назьвать дерево полным, если каждой его финальной вершине соответствует точка $(0,0)$.

Лемма 6. Имеет место разложение

$$
G_{b, p}=\sum_{T} f(T)
$$

где сумма берется по всем полным деревьям. 
ДоКАЗАТЕЛЬСТВО получается полной итерацией рекуррентного соотношения (19), причем мы останавливаемся, только когда не будет финальных точек, отличных от $(0,0)$.

Заметим теперь, что число бинарных вершин в произвольном дереве с корнем $(b, p)$ не превосходит $p$. Действительно, положим $p(T)=\sum p(v)$, где сумма по всем финальным вершинам $v$ дерева $T$. Пусть дерево $T^{\prime}$ получено из $T$ прибавлением двух ребер к некоторой финальной вершине дерева $T$. Тогда $p\left(T^{\prime}\right)=p(T)-1$. Если же дерево $T^{\prime}$ получено из $T$ прибавлением одного ребра к какой-либо финальной вершине дерева $T$, то $p\left(T^{\prime}\right)=p(T)$. Отсюда следует результат.

Лемма 7. Число $K_{b, p}$ nар $(T, \phi)$, где $T$ - полнье деревья с корнем $(b, p)$, не превосходит $C^{b+p}$ для некоторой константы $C$.

ДокАЗАТЕЛЬСтвО. Имеет место рекуррентное соотношение

$$
K_{b, p}=\sum_{j=0}^{p-1} \sum_{k=0}^{b} K_{k, j} K_{b-k, p-j-1}+K_{b-1, p}
$$

Уравнение для производящей функции

$$
K=K(x, y)=\sum K_{b, p} x^{b} y^{p}
$$

имеет вид

$$
K=x K^{2}+y K+1
$$

откуда

$$
K=-\frac{y-1}{2 x}+\frac{y-1}{2 x} \sqrt{1-\frac{4 x}{(y-1)^{2}}} .
$$

Из аналитичности $K$ в точке $x=y=0$ и следует результат.

Согласно последней лемме остается доказать, что для произвольной пары $(T, \phi)$ вклад не превосходит $C^{b+p}$ для некоторой не зависящей от пары константы $C$. Некоторую трудность представляет оценка произведения чисел $2 p / b$ по всем унарным вершинам. Докажем по индукции, что число унарных вершин не более $b$. Начнем с корневой вершины $(b, p)$. Пусть между ней и следуюшей бинарной вершиной ровно $b_{1}$ унарных вершин. Бинарная вершина $\left(b-b_{1}, p\right)$ создает две другие вершины $\left(b^{\prime}, p^{\prime}\right)$ и $\left(b^{\prime \prime}, p^{\prime \prime}\right)$ с $b^{\prime}+b^{\prime \prime}=b-b_{1}, p^{\prime}+p^{\prime \prime}=p-1$. По индукции под этими двумя вершинами не более $b^{\prime}+b^{\prime \prime}$ унарных вершин. Поэтому обшее число бинарных вершин не превосходит $b^{\prime}+b^{\prime \prime}+b_{1}=b$.

Сушествует множество $V^{\prime}$ вершин со следуюшими свойствами: 1) ни одна из вершин $V^{\prime}$ не находится под другой, 2$)$ любая вершина $\left(b^{\prime \prime}, p^{\prime \prime}\right)$, не расположенная под какой-либо из вершин $V^{\prime}$, обладает свойством $\left.b^{\prime \prime} \geqslant 2 p^{\prime \prime}, 3\right)$ для любой вершины $\left(b^{\prime}, p^{\prime}\right)$ из $V^{\prime}$ имеет место $b^{\prime}<2 p^{\prime}$.

Вклад унарных вершин типа 2) будем оценивать как 1 . Возьмем теперь вершину из $V^{\prime}$ с $b^{\prime}<p^{\prime} / \ln p^{\prime}$. Обшее число унарных вершин под ней не превосходит $b^{\prime}$, и поэтому их обший вклад не превосходит $\left(2 p^{\prime}\right)^{p^{\prime} / \ln p^{\prime}}<C^{p^{\prime}}$. 
Пусть теперь $p / \ln p \leqslant b<2 p$ для некоторой вершины $(b, p)$. Обозначим $g(T)=$ $b ! f(T)$, если $(b, p)$ является корнем дерева $T$. Если корень является унарной вершиной с вкладом $2 p / b$, то $g(T)<2 p g\left(T^{\prime}\right)$, так как вершина под ней есть $(b-1, p)$. Если корень является бинарной вершиной с вкладом $\frac{k !(b-k) !}{b !}$, то $g(T)=g\left(T_{1}\right) g\left(T_{2}\right)$, где $T_{i}-$ деревья с корнями $(k, j)$ и $(b-k, p-j-1)$ соответственно для некоторого $j$. Отсюда следует, что $g(T)<(2 p)^{b}$ и, значит,

$$
f(T)<\frac{(2 p)^{b}}{b !}<e^{2 p}
$$

Заметив, что $\sum_{v^{\prime} \in V^{\prime}} p\left(v^{\prime}\right) \leqslant p$, получим результат.

Окончим теперь доказательство теоремы. Рассмотрим функцию одного комплексного переменного

$$
f(z)=\sum_{b=0}^{\infty} z^{b+p(b)} G_{b, p(b)}
$$

По предыдущей лемме ее ряд имеет конечньй радиус сходимости, скажем, $R$. Тогда для любого $\varepsilon>0$ и достаточно больших $b=b(\varepsilon)$ имеет место

$$
\left(R^{-1}-\varepsilon\right)^{b}<G_{b, p(b)}<\left(R^{-1}+\varepsilon\right)^{b}
$$

Отсюда и следует результат.

Представим результат теоремы в более инвариантном виде. Пусть $\rho(M)$ - минимальный род, в которьй можно вложить карту $M$, пусть $\mathfrak{M}(b, p)$ - множество карт с параметрами $b, p$. Положим $\rho(b, p)=\max _{M \in \mathfrak{M}(b, p)} \rho(M)$. Тогда теорема говорит, что $\frac{|\mathfrak{M}(b, p)|}{(2 \rho(b, p)) !}$ имеет экспоненциальньй рост.

Иначе говоря, факториальньй множитель имеет порядок $\left(-\chi_{\min }\right) !$, где $\chi_{\min }$ равно минимуму эйлеровых характеристик получающихся многообразий.

Это видно из следуюшей формулы:

$$
-\chi=2 \rho-2=-V+L-F=b-1-F=-z(P,-)+b-1
$$

где $z(P,-)$ - число орбит групшы, порожденной обеими подстановками. Нетрудно видеть, что при данных орбитах подстановки $P$ можно выбрать подстановку $(-)$ так, чтобы орбиты $(P,-)$ были как угодно велики, т.е. $z(P,-)$ давало вклад гораздо меньше $b$.

Данньй результат позволяет предложить разумное каноническое распределение на множестве $\mathscr{A}(b)$ всех карт $M$ с фиксированным $b=-\chi_{\min }$ :

$$
P_{b}(M)=Z_{b}^{-1} \exp (-\mu p(M)-\lambda \rho(M))=Z_{b}^{-1} \exp \left(-\mu p(M)+\frac{\lambda}{2} F(M)\right) .
$$


3.2. Размерности 3 и выше. В квантовой гравитации при заданном триангулируемом $d$-мерном многообразия $M$ (симплициальном комплексе) потенциал считается линейным по числу $N_{i}(T) i$-мерных симплексов триангуляции $T, i=0, \ldots, d$, т.е. статсумма равна формально

$$
Z=\sum_{T} \exp \left(-\sum_{i} k_{i} N_{i}(T)\right)=\sum_{\left\{N_{i}\right\}} \exp \left(-\sum_{i} k_{i} N_{i}\right) C\left(\left\{N_{i}\right\}\right),
$$

где $C\left(\left\{N_{i}\right\}\right)$ - число триангулящий с заданными числами $\left\{N_{i}\right\}$. Но ввиду соотношений Дэна-Зоммерфельда (см. [12]) все $N_{i}(T)$ линейно выражаются через два из них. Поэтому можно записать распределение в виде

$$
\sum_{T} \exp \left(-\lambda_{d} N_{d}(T)+\lambda_{d-2} N_{d-2}(T)\right)
$$

где сумма по всем триангулящиям $T$ заданного многообразия. Более того, последняя формула соответствует действию Гильберта-Эйнштейна

$$
S=\frac{1}{16 \pi G} \int d x \sqrt{g}(2 \Lambda-R)
$$

где $R$ - скалярная кривизна, $\Lambda$ - космологическая константа, $G$ - константа Ньютона. Для сходимости ряда в некоторой области параметров необходимы экспоненциальные оценки на числа $C\left(\left\{N_{i}\right\}\right)$. Обсуждение необходимых экспоненциальных оценок см. в [12]. Д. Булатов [13] приводит следуюший результат. Пусть $C(N, \mathbf{M})$ - число 3 -мерных триангуляций с $N$ симплексами трехмерного замкнутого многообразия $\mathbf{M}$. Тогда

$$
\sum C(N, \mathbf{M})<C \exp (\gamma N)
$$

где сумма берется по всем $\mathbf{M}$ с фиксированной группой гомологий $H_{1}(\mathbf{M}, Z), \gamma=$ $\gamma\left(H_{1}(\mathbf{M}, Z)\right)$.

В обшем случае (если топология произвольна) энтропия неэкспоненциальна, и единственным известным автору способом ввести вероятностное распределение являются гиббсовские семейства.

Каждому $d$-мерному симплициальному комплексу соответствует двойственньй граф, вершины которого соответствуют $d$-мерным симплексам. Каждая вершина этого граффа имеет $d$ отростков, соответствуюших $d$ его $(d-1)$-мерным граням. Спариванию ног соответствует склеивание соответствуюших граней с помощью линейного отображения. В размерности 2 при таком склеивании обязательно получается многообразие. В больших же размерностях при склеивании граней $d$-мерных клеток с вероятностью, стремяшейся к 1, получится псевдомногообразие. Более того, при термодинамическом предельном переходе получаемое псевдомногообразие не имеет ни одной вершины с окрестностью, изоморфной шару. Это следует из результата раздела 4.4 .

Условие, выделяющее многообразия среди псевдомногообразий, является локальным и может быть задано суперлокальньм потенциалом (с твердой сердцевиной). Однако ограничение на топологию не является локальным условием. Ниже мы рассмотрим простейшие примеры, где ограничение на топологию получается как следствие гиббсовости. 


\section{4. Фазовые переходы}

Гиббсовское семейство (на $\mathscr{A}_{\infty}^{(0)}$ или на $\mathscr{A}_{\infty}$ ) с потенциалом $\Phi$ назьвается чистым гиббсовским семейством, если оно не является вьпуклой комбинацией гиббсовских семейств с тем же потенциалом. Таким образом, неединственность гиббсовского семейства означает, что есть по крайней мере два чистых гиббсовских семейства с тем же потенциалом $\Phi$. Чистое гиббсовское семейство может быть двух типов: сосредоточенное на фиксированном графе или имеющее носитель на нескольких или даже континууме различных графов. В первом случае оно сводится, таким образом, к обычному гиббсовскому полю.

Соответственно, неединственность гиббсовских семейств может иметь различньй характер. Во-первых, на каждом графе из носителя гиббсовского семейства чистые фазы могут отличаться структурой типичных конфигураций (фазовьй переход, наследуемьй из обычных гиббсовских полей на фиксированном графе). Во-вторых, чистые фазы могут отличаться по типичной структуре самого графа (метрический или топологический фазовый переход). Нестрого говоря, топологический фазовый переход имеет место, если имеется по крайней мере два гиббсовских семейства с непересекающимися носителями, и при этом носители имеют графы (с локальной структурой, определяющей топологическое пространство) с существенно разной топологией.

Вопрос о полном описании всех фаз достаточно сложен. Проше выделить какие-то чистые фазы, что мы и делаем ниже для нескольких примеров. Для этого бывает полезно следуюшее простое замечание: если чистое гиббсовское поле есть гиббсовское семейство, то оно является чистым гиббсовским семейством.

4.1. Модель среднего поля. В дискретной квантовой гравитации есть простая модель [17], где аналитически можно объяснить появление разных фаз в симплициальной гравитации со спином при численном моделировании. Это урновая модель с $M=\lambda N$ шарами в $N$ урнах. Ее можно интерпретировать как случайный граф, если урны назвать вершинами, а шары - отростками, затем отростки случайно соединяются попарно. Таким образом, имеется $N$ занумерованных вершин $i=1, \ldots, N$ с $q_{i} \geqslant 1$ отростками у вершины $i$. Канонический ансамбль задается статсуммой

$$
Z_{N}=\sum_{\left\{q_{i}\right\}: \sum q_{i}=M} \exp \left(-\sum_{i=1}^{N} \beta \Phi\left(q_{i}\right)\right) .
$$

Заметим, что в нашей терминологии это модель с локальным потенщиалом (неустойчивьм, как было показано вьше) и с нелокальным ограничением $\sum q_{i}=M=\lambda N$ типа среднего поля. Данное ограничение устраняет неустойчивость таких локальных потенциалов. Заметим, что факториальньй множитель $A(M)$ в асимптотике статсуммы, отвечаюший за случайное спаривание отростков, вьносится, после чего асимптотика статсуммы принимает вид

$$
Z_{N}=c N^{\alpha} \exp (\gamma N)
$$

Для довольно широкого класса потенциалов, в том числе и для $\Phi(q)=\ln q$, имеется две фазы с фазовым переходом по $\lambda$ при фиксированном $\beta$. Одна фаза (elongated, fluid) для малых плотностей, где шары равномерно распределены по урнам, вторая 
(crumpled, Bose-Einstein) фаза - где в одной единственной вершине сосредоточено порядка $M$ шаров. Некоторым видоизменением модели можно получить и смешанную фазу (condensed, crinkled), где есть одна вершина с $c M, c<1$, шарами, а остальные урны заполнены равномерно.

Численное моделирование, см. [18], показывает возникновение аналогичных фаз в двумерной симплициальной гравитации при добавлении спинов. Поэтому интересны различные объяснения и аналоги таких фаз. Как уже говорилось, это - сглаженньй переход между устойчивостью и неустойчивостью, сглаживание обеспечивается ограничением среднего поля.

В физике аналогом является конденсация Бозе-Эйнштейна. В информатике выбор $\Phi=c q$ дает стационарное распределение сетей Джексона в модели среднего поля, см. [19], где наблюдаются совершенно аналогичные фазовые переходы. Вся классическая теория случайных графов имеет дело с химическим потенциалом при таком же ограничении типа среднего поля, см. [20].

4.2. Фазовые переходы в модели с границами. Мы рассматриваем двумерные триангулящии $T$, назьваемые почти-триангулящиями в [21] и триангуляциями в [11]. Иначе говоря, мы рассматриваем вложения граффа в двумерную поверхность, где ребра - непересекающиеся гладкие дуги, а дополнение состоит из непересекающихся открытых областей, гомеоморфных диску, причем каждая область ограничена ровно тремя дугами.

Пусть $T\left(\rho, N, k ; m_{1}, \ldots, m_{k}\right)$ - множество всех (классов эквивалентности) таких триангулящй сферы с $\rho$ ручками, $k$ дырками, $N$ треугольниками и $m_{i}$ ребрами на гранище $i$-й дырки. Мы считаем, что $m_{i} \geqslant 2$. Пусть $C\left(\rho, N ; m_{1}, \ldots, m_{k}\right)$ - число таких триангулящий. При фиксированных $\rho$ и $k$ для данного $N$ определим распределение $\mu_{\rho, N, k}$ (канонический ансамбль) на множестве триангуляций

$$
T(\rho, N, k)=\bigcup_{m_{1}, \ldots, m_{k}} T\left(\rho, N, k ; m_{1}, \ldots, m_{k}\right)
$$

Мы введем распределение на $T(\rho, N, k)$ четьрьмя эквивалентными способами:

• где взаимодействие пропорционально общему числу ребер, т.е.

$$
\mu_{\rho, N, k}(T)=Z_{N}^{-1} \exp \left(-\mu_{1} L(T)\right)
$$

• где взаимодействие пропорционально сумме степеней вершин, ибо

$$
\exp \left(-\mu_{1} L(T)\right)=\exp \left(-\frac{\mu_{1}}{2} \sum_{v \in V(T)} \operatorname{deg} v\right)
$$

здесь $L(T)$ - число ребер, включая граничные, у триангуляции $T$. Поэтому

$$
Z_{N}=Z_{N}(\rho, k)=\sum_{\left(m_{1}, \ldots, m_{k}\right)} \sum_{T \in T\left(\rho, N ; m_{1}, \ldots, m_{k}\right)} \exp \left(-\frac{\mu_{1}}{2} \sum_{v \in V(T)} \operatorname{deg} v\right) .
$$


Используя дискретньй аналог теоремы Гаусса-Бонне, можно показать, что это дискретньй аналог действия Гильберта-Эйнштейна. Заметим, что это частный случай (с параметрами $t_{q}=t$ ) взаимодействия, рассмотренного в работе [22]:

$$
\prod_{q>2} t_{q}^{n(q, T)}
$$

где $n(q, T)$ - число вершин степени $q$.

- где взаимодействие пропорционально числу граничных peбер, т.е. для всех $\rho$ и $k$ определим коррелящионные функции векторов $\left(m_{1}, \ldots, m_{k}\right)$

$$
\begin{aligned}
P_{N, \rho, k}\left(m_{1}, \ldots, m_{k}\right) & =\sum_{T \in T\left(\rho, N ; m_{1}, \ldots, m_{k}\right)} \mu_{\rho, N, k}(T) \\
& =\Theta_{N}^{-1}(\rho, k) \exp \left(-\mu_{1} \frac{\sum_{i=1}^{k} m_{i}}{2}\right) C\left(\rho, N ; m_{1}, \ldots, m_{k}\right) .
\end{aligned}
$$

Последнее равенство следует из формулы

$$
L(T)=\frac{3 N-\sum m_{i}}{2}+\sum m_{i}=\frac{3 N}{2}+\frac{\sum m_{i}}{2} .
$$

Значит,

$$
\Theta_{N}(\rho, k)=\sum_{m_{1}, \ldots, m_{k}=2}^{\infty} \exp \left(-\mu_{1} \frac{\sum m_{i}}{2}\right) C\left(\rho, N ; m_{1}, \ldots, m_{k}\right)
$$

- Введем спины $\sigma_{v}$ в вершинах $v$ триангулящии, они принимают значения в произвольном компактном множестве и соответствуюший потенциал гиббсовского семейства имеет вид

$$
f\left(\sigma_{v}, \sigma_{v^{\prime}}\right) \equiv 1
$$

для любых двух соседних вершин $v, v^{\prime}$.

Рассмотрим сначала случай $k=1, \rho=0$. Более того, предположим, что на границе выделена вершина и инцидентное ей ребро, определяя таким образом начало системы координат и ориентацию. Обозначим класс таких триангулящий $T_{0}(N, m)$, а их число $C_{0}(N, m)$. Вероятность триангуляций здесь равна

$$
P_{0, N}(T)=Z_{0, N}^{-1} \exp \left(-\frac{\mu_{1}}{2} \sum_{v \in V(T)} \operatorname{deg} v\right)
$$

Мы докажем существование фазового перехода по $\mu_{1}$. Обозначим $\mu_{1, \mathrm{cr}}=\ln 12$. Пусть $0<\beta_{0}=\beta_{0}\left(\mu_{1}\right)<1$ определяется из уравнения

$$
\frac{1+\frac{4 \beta_{0}}{3\left(1-\beta_{0}\right)}}{\left(1+\frac{2 \beta_{0}}{1-\beta_{0}}\right)^{2}} \exp \left(-\mu_{1}+\ln 12\right)=1
$$


Теорема 5. Свободная әнергия $\lim _{N} \frac{1}{N} \ln Z_{0, N}=F$ равна $-\frac{3}{2} \mu_{1}+c, c=3 \sqrt{\frac{3}{2}}$, если $\mu_{1}>\mu_{1, \mathrm{cr}}$, и равна

$$
-\frac{3}{2} \mu_{1}+c+\beta_{0}\left(-\mu_{1}+\ln 12\right)+\int_{0}^{\beta_{0}} \ln \frac{1+\frac{4 \beta}{3(1-\beta)}}{\left(1+\frac{2 \beta}{1-\beta}\right)^{2}} d \beta,
$$

если $\mu_{1}<\mu_{1, \mathrm{cr}}$.

Заметим, что $\mu_{1} \rightarrow \mu_{1, c r}$, если $\beta_{0} \rightarrow 0$.

Пусть $m(N)$ - случайная длина границы при фиксированном $N$. Ее распределение имеет вид (если заметить, что $|L(T)|=3 N / 2+m / 2)$ :

$$
\begin{aligned}
P_{0, N}(m(N) & =m)=\Theta_{0, N}^{-1} \exp \left(-\mu_{1} \frac{m}{2}\right) C_{0}(N, m), \\
\Theta_{0, N} & =\sum_{m} \exp \left(-\mu_{1} \frac{m}{2}\right) C_{0}(N, m) .
\end{aligned}
$$

Теорема 6. Существуют три фазы, в которых распределение $m(N)$ имеет весьма разное асимптотическое поведение

- Субкритическая фаза, т.е. $12 \exp \left(-\mu_{1}\right)<1: m(N)=O(1)$, точнее, распределение $m(N)$ имеет предел $\lim _{N} P_{0, N}(m(N)=m)=p_{m}$ для фиксированных $m$ при $N \rightarrow \infty$.

- Суперкритическая фаза, т.е. $12 \exp \left(-\mu_{1}\right)>1$. Здесь длина границы имеет порядок $O(N)$. Точнее, имеется $\varepsilon>0$ такое, что $\lim P_{0, N}\left(m_{N} / N>\varepsilon\right)=1$.

- В критической точке, т.е. $12 \exp \left(-\mu_{1}\right)=1$, длина граничы имеет порядок $\sqrt{N}$. Точнее, распределение $m_{N} / \sqrt{N}$ сходится по вероятности.

ДокАЗАТЕЛЬСТВО. Мы используем формулу $(N=m+2 j)$

$$
C_{0}(N, m)=\frac{2^{j+2}(2 m+3 j-1) !(2 m-3) !}{(j+1) !(2 m+2 j) !((m-2) !)^{2}}
$$

(см. [21]). Прямьм вычислением, принимая во внимание, что замена $N \rightarrow N$, $m \rightarrow m+2$ соответствует замене $j \rightarrow j-1, m \rightarrow m+2$, получаем

$$
\begin{aligned}
& \frac{P_{0, N}(m+2)}{P_{0, N}(m)}=f(N, m)=\frac{C_{0}(N, m+2) \exp \left(-\frac{\mu_{1}}{2}(m+2)\right)}{C_{0}(N, m) \exp \left(-\frac{\mu_{1}}{2} m\right)} \\
& \quad=\exp \left(-\mu_{1}+\ln 12\right) \frac{\left(1+\frac{2}{N-m}\right)\left(1+\frac{4 m}{3(N-m)}\right)}{\left(1+\frac{2 m}{N-m}+\frac{2}{N-m}\right)\left(1+\frac{2 m}{N-m}+\frac{1}{N-m}\right)} \frac{\left(1-\frac{1}{4 m^{2}}\right)}{\left(1-\frac{1}{m}\right)} .
\end{aligned}
$$

В субкритическом случае при фиксированном $m$ и $N \rightarrow \infty$

$$
\frac{P_{0, N}(m+2)}{P_{0, N}(m)} \sim \exp \left(-\mu_{1}+\ln 12\right)\left(1+\frac{1}{m}+O\left(\frac{1}{m^{2}}\right)\right)
$$


и при $m \rightarrow \infty$, например, для четных $m$,

$$
\lim _{N} P_{0, N}(2 m) \sim C m \exp \left(m\left(-\mu_{1}+\log 12\right)\right) .
$$

В то же время второй множитель в правой части (21) не превосходит 1. Поэтому из

$$
Z_{0, N}=\exp \left(-\mu_{1} \frac{3 N}{2}\right) \Theta_{0, N}=\exp \left(-\mu_{1} \frac{3 N}{2}\right) \sum_{m} \exp \left(-\mu_{1} \frac{m}{2}\right) C_{0}(N, m)
$$

получаем $F=-\frac{3}{2} \mu_{1}+c, c=3 \sqrt{\frac{3}{2}}$, так как для фиксированного $m$ имеем

$$
C_{0}(N, m) \sim \phi(m) N^{-\frac{5}{2}} c^{N} .
$$

Из (21) получаем (20) также и в суперкритическом случае, если положить $m=\beta N$, $0<\beta<1$. В критической точке оба выражения совпадают, но свободная энергия не дифференцируема в этой точке.

Для доказательства второго утверждения теоремы положим $12 \exp \left(-\mu_{1}\right)=1+r$ и оценим отдельно каждый из трех множителей в (21). Тогда получим, что существуют $0<\delta \ll \varepsilon \ll 1$ такие, что для всех $m \leqslant \delta N$

$$
\frac{P_{0, N}(m)}{P_{0, N}(\varepsilon N)}<\left(1+\frac{r}{2}\right)^{-\frac{\varepsilon}{2} N}
$$

Отсюда и следует результат.

В критической точке аналогично имеем

$$
\frac{P_{0, N}(m+2)}{P_{0, N}(2)} \sim \prod_{k=1}^{m / 2}\left(1+\frac{1}{2 k}\right)\left(1-\frac{4}{3} \frac{k}{N}\right) \sim C \sqrt{m} \exp \left(-\frac{1}{3} \frac{m^{2}}{N}\right) .
$$

Отсюда для любого $0<\alpha<\beta<\infty$

$$
\lim \left(\frac{P_{0, N}(m(N)<\varepsilon \sqrt{N})}{P_{0, N}(\beta \sqrt{N}<m(N)<\beta \sqrt{N})}+\frac{P_{0, N}\left(m(N)>\varepsilon^{-1} \sqrt{N}\right)}{P_{0, N}(\beta \sqrt{N}<m(N)<\beta \sqrt{N})}\right)=0
$$

при $\varepsilon \rightarrow 0$.

Рассмотрим теперь случай, когда на границе нет выделенной вершины, иначе говоря, мы расширяем группу автоморфизмов. Свободная энергия, как и поведение вне критической точки, не меняется.

Теорема 7. В критической точке, без системы координат на граниче, длина граничы имеет порядок $N^{\alpha}, 0<\alpha<1 / 2$. Точнее, распределение $\frac{\log m_{N}}{\log \sqrt{N}}$ сходится к равномерному распределению на единичном интервале, т.е. $P_{0, N}\left(\frac{\alpha}{2} \leqslant \frac{\log m_{N}}{\log \sqrt{N}} \leqslant \frac{\beta}{2}\right) \rightarrow \beta-\alpha$ для всех $0 \leqslant \alpha<\beta \leqslant 1$. 
Лемма 8. $C(N, m) \sim m^{-1} C_{0}(N, m)$ nрu $N, m \rightarrow \infty$.

ДокАЗАТЕльСтвО ЛЕммы. Перенумеруем в циклическом порядке ребра границы: $1,2, \ldots, m$, начиная с корневого. Автоморфизм диска $\phi$ однозначно определен, если задано ребро $j=\phi(1)$, в которое переходит ребро с номером 1. Действительно, тогда примыкающие к ребру 1 треугольники однозначно переходят в треугольники, примыкающие к $j$, и так далее по связности.

Рассмотрим полосу ширины 1, примыкаюшую к границе, т.е. множество треугольников трех типов: которые имеют с границей общую сторону (тип 1), две обшие стороны (тип 2) и те, которые имеют с ней только общую точку (тип 0). Таким образом, полоса может быть представлена в виде слова $\alpha=x_{1} \ldots x_{n}, n>m$, где $x_{i}=0,1,2$. Рассмотрим множество $W\left(m, n_{0}, n_{1}, n_{2}\right)$ слов с заданными $m$ и числами $n_{i}$ букв $i$. Автоморфизм диска дает циклический автоморфизм слова $\alpha$. При этом множества $W\left(m, n_{0}, n_{1}, n_{2}\right)$ инвариантны. Заметим, что

$$
m=n_{1}+2 n_{2}
$$

и длина другой гранищы полосы равна $m^{\prime}=n_{0}$. Поэтому, если не существует циклического автоморфизма слова $\alpha$, то нет и автоморфизмов диска. Легко видеть, что при заданном $n_{0}$ и при $m \rightarrow \infty$ множество слов из $\bigcup_{n_{1}, n_{2}} W\left(m, n_{0}, n_{1}, n_{2}\right)$, имеющих нетривиальные циклические автоморфизмы, мало в сравнении с общим числом $\left|\bigcup_{n_{1}, n_{2}} W\left(m, n_{0}, n_{1}, n_{2}\right)\right|$.

Это дает в $f(N, m)$ множитель $1-1 / m$ вместо $1+1 / m$ в предыдущем случае. Аналогичные вычисления доказывают, что асимптотически распределение совпадает с семейством $\nu_{N}$ распределений на множестве $\{1, \ldots, \sqrt{N}\}$ из $\sqrt{N}$ элементов

$$
\nu_{N}(i)=Z_{\sqrt{N}} i^{-1}, \quad Z_{\sqrt{N}}=\sum_{i=1}^{\sqrt{N}} i^{-1}
$$

Легко видеть, что для $0 \leqslant \alpha \leqslant \beta \leqslant 1$

$$
\nu_{N}\left(\frac{\alpha}{2} \leqslant \frac{\log i}{\log \sqrt{N}} \leqslant \frac{\beta}{2}\right)=\nu_{N}\left(N^{\alpha / 2} \leqslant i \leqslant N^{\beta / 2}\right) \rightarrow \beta-\alpha .
$$

4.2.1. Связь с топологической теорией поля. Введение системы координат на границе напоминает переход от топологической теории поля к конформной теории поля. Топологическая теория поля (ТТП) обычно вводится аксиоматически в рамках теории категорий [23], эвристически (с помощью интегралов по путям) или алгебраически (как алгебра Фробениуса). Чтобы придать смысл интегралу по путям, идея дискретизации (см., например, [24]-[26]) кажется весьма естественной. В последней главе [27] строится ТТП, которая удовлетворяет всем аксиомам уже на конечных триангулящиях. Однако в принщипе возможны случаи, когда она удовлетворяет им только в пределе $N \rightarrow \infty$. Исследовать этот предел - задача интересная сама по себе. Мы здесь делаем это для случая двух границ (т.е. $k=2$ ), нулевого рода и для больших $\mu_{1}$ в предыдушей модели. Интересно, что возникает нетривиальное совместное распределение длин границ. 
Теорема 8. Для $k=2$ при достаточно больших $\mu_{1}$ и фиксированных $m_{1}, m_{2}$ существуют корреляиионные функиии $P_{0,2}\left(m_{1}, m_{2}\right)=\lim _{N} P_{N, 0,2}\left(m_{1}, m_{2}\right)$.

Мы приводим доказательство полностью, аналогичньй метод по-видимому работает для всех $k$ и $\rho$. Получим сначала следуюшие рекуррентные соотношения для чисел $C_{0}\left(N, m, m_{2}\right), m=m_{1}$ :

$$
\begin{aligned}
C_{0}\left(N, m, m_{2}\right)= & C_{0}\left(N-1, m+1, m_{2}\right)+C_{0}\left(N-1, m+m_{2}+1\right) \\
& +\sum_{N_{1}+N_{2}=N-1} \sum_{k_{1}+k_{2}=m+1} C_{0}\left(N_{1}, k_{1}, m_{2}\right) C_{0}\left(N_{2}, k_{2}\right) .
\end{aligned}
$$

Они получаются, как и в методе Татта [28], удалением корневого ребра. См. рис. 1.

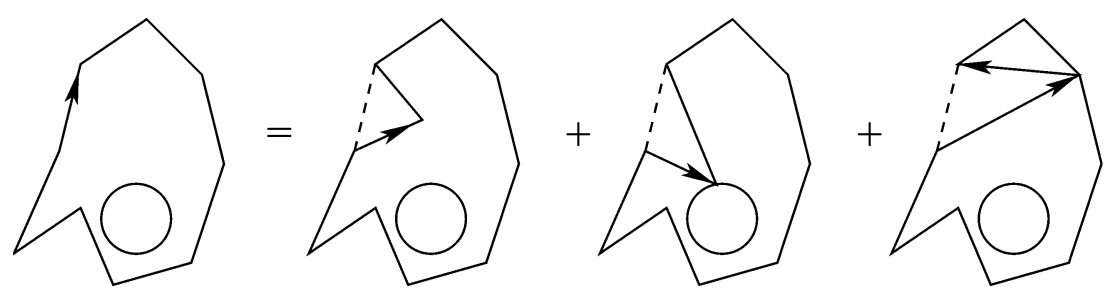

Рис. 1

Таким образом, возможны три случая, соответствуюшие трем членам правой части: граф̆ становится несвязньм; две дырки сливаются в одну; остаются две дырки, но у первой из них граница удлиняется, а число треугольников уменьшается. Граничные условия таковы. Для заданного $N$ только конечное число $C_{0}\left(N, m, m_{2}\right) \neq 0$, и удобно считать $C_{0}\left(N, m, m_{2}\right)$ известньми для $N<N_{0}$, их явньй вид нас не интересует. Кроме того, $C_{0}\left(N, m, m_{2}\right)=0$, если либо $m<2$, либо $m_{2}<2$.

Лемма 9. Асимптотика при $N \rightarrow \infty$ и фиксированных $m, m_{1}$ такова:

$$
C_{0}\left(N, m, m_{2}\right) \sim \phi\left(m, m_{2}\right) N^{-5 / 2+1} c^{N} .
$$

При этом $\phi(m, 3)=\phi(m)$.

ДОКАЗАТЕЛЬСтво. Хорошо известно, что

$$
C_{0}(N, m) \sim \phi(m) N^{-5 / 2} c^{N} .
$$

Найдем $C_{0}(N, m, 2), C_{0}(N, m, 3), C_{0}\left(N, 2, m_{2}\right), C_{0}\left(N, 3, m_{2}\right)$. Пусть сначала $m_{2}=3$. Чтобы получить триангулящию из $T(0, N ; m, 3)$, возьмем триангулящию из $T(0, N+1$; $m)$ и выберем один из $N$ треугольников так, чтобы он не касался гранишы, что можно сделать $N-O(m)$ способами. Отсюда следует, что $C_{0}(N, m, 3) \sim C_{0}(N+1, m) N$, и, значит, $\phi(m, 3)=c \phi(m)$. Убирая корень на границе $m$ и помещая его на границу длины 3 , имеем из тривиальности групшы автоморфизмов для почти всех графов, что $\phi(3, m)=\frac{3 c}{m} \phi(m)$. Чтобы получить триангуляцию из $T(0, N ; m, 2)$, возьмем триангулящию из $T(0, N ; m)$, выберем один из $N$ треугольников так, чтобы он не 
касался границы, выберем одну из его сторон и раздвоим ее. Тогда $\phi(m, 2)=\frac{3}{2} \phi(m)$ и аналогично $\phi(2, m)=\frac{3}{m} \phi(m)$.

Перепишем рекуррентные соотношения:

$$
\begin{aligned}
C_{0}\left(N-1, m+1, m_{2}\right)= & C_{0}\left(N, m, m_{2}\right) \\
& -\sum_{\substack{N_{1}+N_{2}=N-1\\
}} \sum_{k_{1}+k_{2}=m+1} C_{0}\left(N_{1}, k_{1}, m_{2}\right) C_{0}\left(N_{2}, k_{2}\right) \\
& -C_{0}\left(N-1, m+m_{2}+1\right) .
\end{aligned}
$$

Так как мы знаем $C_{0}(N, m, 2), C_{0}(N, m, 3)$ и $C_{0}\left(N, 2, m_{2}\right), C_{0}\left(N, 3, m_{2}\right)$, то результат следует индукцией по $m$ при фиксированном $m_{2}$ и наоборот. Действительно, умножим это соотношение на $c^{-N+1}(N-1)^{5 / 2-1}$. Тогда по индуктивному предположению правая часть стремится к

$$
c \phi\left(m, m_{2}\right)-2 \sum_{k_{1}+k_{2}=m+1} \phi\left(k_{1}, m_{2}\right) \sum_{n=1}^{\infty} C_{0}\left(n, k_{2}\right) c^{-n} .
$$

Поэтому предел левой части $c^{N-1}(N-1)^{5 / 2-1} C_{0}\left(N-1, m+1, m_{2}\right)$ конечен, обозначим его $\phi\left(m+1, m_{2}\right)$.

Перейдем к доказательству теоремы. Подставляя (23) в рекуррентную формулу для $C_{0}(N, m)$, получим рекуррентную формулу для $\phi(m)$ :

$$
\phi(m+1)=c \phi(m)-2 \sum_{k_{1}+k_{2}=m+1} \phi\left(k_{1}\right) \sum_{n} C_{0}\left(n, k_{2}\right) c^{-n} .
$$

В частности,

$$
\phi(3)=c \phi(2), \quad \phi(4)=c \phi(3)-2 \phi(2) \sum_{n} C_{0}(n, 2) c^{-n} .
$$

Получим теперь асимптотику для $\phi\left(m, m_{2}\right)$ из соотношения

$$
\phi\left(m+1, m_{2}\right)=c \phi\left(m, m_{2}\right)-2 \sum_{k_{1}+k_{2}=m+1} \phi\left(k_{1}, m_{2}\right) \sum_{N_{2}=1}^{\infty} C_{0}\left(N_{2}, k_{2}\right) c^{-N_{2}} .
$$

Отсюда $\phi\left(m, m_{2}\right)<\phi\left(2, m_{2}\right) c^{m}$. Но $\phi\left(2, m_{2}\right)=\frac{2}{m_{2}} \phi\left(m_{2}, 2\right)<\phi(2,2) c^{m_{2}}$. Теорема доказана.

4.3. Неединственность в модели с низкой энтропией. Пусть $U_{2}-$ граф, изоморфный 2-окрестности начала координат решетки $\mathbb{Z}^{2}$. Тогда среди графов, принадлежаших $\mathscr{G}\left(U_{2}\right)$, есть сама решетка $\mathbb{Z}^{2}$ и ее факторгруппы $\mathbb{Z}\left(k_{1}, 0\right), \mathbb{Z}\left(k_{1}, k_{2}\right)$ относительно подгрупп $\left\{\left(n k_{1}, 0\right)\right\}$ и $\left\{\left(n_{1} k_{1}, n_{2} k_{2}\right)\right\}, n, n_{1}, n_{2} \in \mathbb{Z}$, т.е. цилиндры и торы соответственно, где $k_{i} \geqslant 4$. Заметим, что есть и другие, например, скрученные цилиндры, получающиеся из полосы $\mathbb{Z} \times\{0,1,2, \ldots, k\} \subset \mathbb{Z}^{2}$ отождествлением точек $(n, 0)$ и $(n+j, k)$ для каждого $n$. Если нет спина, то рост энтропии здесь субэкспоненциальньй.

Рассмотрим гиббсовское семейство с $S=\{-1,1\}$ и потенциалом $\Phi$, равньм сумме $\Phi_{U_{d, 2}}$ и потенщиала Изинга взаимодействия ближайших соседей $\Phi_{\mathrm{Is}}=\sum_{\langle i, j\rangle} \sigma_{i} \sigma_{j}$. 
Теорема 9. Для любого $\beta$ существует бесконечное множество чистых гиббсовских семейств с потенииалом $\Phi$, которые являются гиббсовскими полями на фиксированных графах (иилиндрах).

ДокАЗАТЕЛЬСтво. Построим (для достаточно большого $N$ ) конечное гиббсовское семейство с граничными условиями, задаваемыми графом $\gamma_{N}$, определяемым следуюшим образом. Рассмотрим подграф $\alpha_{n}$ графа $\mathbb{Z}^{2}$ - полосу, состоящую из точек $(i, j), i \in \mathbb{Z}, j=-n, \ldots, n, n \geqslant 2$, с корнем $(0,0)$. При этом точки $(i,-n)$ и $(i, n)$ отождествлены. Расстояние точки $(i, n)$ от $(0,0)$ определяется как $|i|+|n|$. Положим $\gamma_{N}=\gamma\left(\alpha_{n},(0,0) ; N+1, N+3\right)$. Если $n \ll N$, то $\gamma_{N}$ состоит из двух изоморфных связных компонент. При этом мы воспользуемся леммой 2 , считая, что $\nu_{N}-$ единичная мера на спинграфе $\gamma_{N}$, причем все спины на $\gamma_{N}$ равны 1 . Мы докажем, что (конечное) гиббсовское семейство на $\mathscr{G}_{N}^{(0)}$ будет гиббсовской мерой (Изинга) на графе $\gamma\left(\alpha_{n},(0,0) ; 0, N\right)$.

Для доказательства удобно сначала считать, что $S$ тривиально, используя только часть потенциала, равную $\Phi_{U_{d, 2}}$. Докажем, что искомое конечное гиббсовское семейство с определенньми граничньми условиями будет единичной мерой на $\gamma\left(\alpha_{n},(0,0)\right.$; $0, N)$, т.е. случайньй граф $G$ является с вероятностью 1 графом $\gamma\left(\alpha_{n},(0,0) ; 0, N\right)$. Введем метод "аналитического" продолжения, состояший в индуктивном доказательстве того, что слои $\gamma(N)=\gamma(G, 0 ; N, N), \gamma(N-1)$ и т. д. случайного графа $G$ совпадают с соответствуюшими слоями графа $\alpha_{n}$. Построим сначала слой $\gamma(N)$ и докажем, что он единственен и совпадает с соответствующим слоем $\gamma\left(\alpha_{n},(0,0) ; N, N\right)$ графра $\alpha_{n}$.

Рассмотрим точку $(N+2,0)$ в $\gamma_{N}$ и ее 2-окрестность в $\gamma_{N}$. Этой окрестности не хватает одного ребра $l_{0}$ из точки $(N+1,0)$ с новой вершиной $v_{0}$, которую обозначим $(N, 0)$. Последняя вершина должна быть на расстоянии $N$ от начала, так как все точки на расстояниях $N+1, N+2 ; N+3$ уже даны.

Рассмотрим теперь точку $(N+1,1)$. Из вида ее окрестности в $\gamma\left(\alpha_{n},(0,0) ; N+1\right.$, $N+3)$ следует, что из точки $(N, 1) \in \gamma\left(\alpha_{n} ; N+1, N+3\right)$ должно выходить два новых ребра $l_{1}, l_{2}$ на слой $N$. При этом одно из них должно быть соединено с вершиной $v_{0}$.

Далее по индукции строим весь слой $\gamma(N)$ (и только его), сначала точки с положительной первой координатой, а затем симметричные. При этом все точки слоя $\gamma(N+2)$ получат нужные окрестности и можно исключить их из дальнейшего рассмотрения.

Далее индукцией по слоям строим слои $\gamma(N-1), \gamma(N-2), \ldots$, используя окрестности уже построенных слоев $N+1,-N-1, N,-N, \ldots$ Э Эа индукция действует до тех пор, пока две связные компоненты не сольются в одну. Докажем, что это событие произойдет на прямой $y= \pm n$ на слое $n$. Исключим две возможности: первая слияние произойдет впервые на другой прямой, вторая - оно произойдет на прямой $y= \pm n$, но на слое, отличном от слоя $n$. Вторая возможность исключается следуюшими рассуждениями. После слияния на прямой $y= \pm n$ образовавшаяся полость заполняется ровно за $n$ шагов индукции. Поэтому слияние должно произойти именно на слое $n$. Первая возможность устраняется так. Предположим, что слияние произошло впервые на прямой $y=k$. Тогда прямая $y=k+1$ на этом же шаге индукции будет иметь препятствие к заполнению.

Таким образом, вне зависимости от спинов на $\gamma_{N}$ гиббсовское семейство имеет носитель на единственном графе, и, тем самым, данное гиббсовское семейство является гиббсовским полем. Теорема доказана, так как $n$ можно выбрать произвольно. 


\section{4. Гиббсовская характеризация структур.}

4.4.1. Единственность в модели с высокой энтропией. Счетные деревья нельзя характеризовать локально, т.е. они не могут быть выделены из класса всех графов каким-либо ограничением на подграфы диаметра меньшего некоторой константы. Интересно, однако, что локальная характеризация счетных деревьев может быть дана с помощью гиббсовских семейств с локальным потенциалом. Точнее, можно определить гиббсовские семейства с потенциалом диаметра 2 и имеющие носитель на множестве счетных деревьев. Мы рассматриваем здесь случай $p$-регулярных деревьев.

Кроме того, это дает пример, когда статсумма имеет факториальный рост, но тем не менее гиббсовское семейство единственно.

Рассмотрим множество $\mathscr{A}_{N, p}$ всех $p$-регулярных графов с $N$ вершинами, т.е. графов, в которых все вершины имеют степень $p \geqslant 3$. Рассмотрим конечное гиббсовское семейство на $\mathscr{A}_{N}$ со следуюшим суперпотенциалом $\Phi: \Phi=0$ на графах радиуса 1 , если 0 имеет степень $p$, и $\Phi=\infty$ для остальных графов радиуса 1 . Можно сказать также, что рассматривается конечное гиббсовское семейство $\mu_{N}$ на $\mathscr{A}_{N, p}$ с потенциалом $\Phi \equiv 0$.

ТеОрема $10 . \lim p^{N}\left(\Gamma_{k}\right)=p\left(\Gamma_{k}\right)=1$, если $\Gamma_{k}-$ любое р-регулярное дерево, в котором любой путь из 0 в финальную вершину имеет длину $k$, и 0 для осталь$\operatorname{Hblx} \Gamma_{k}$.

ДокАЗАТЕЛьство. Заметим, что все графы из $\mathscr{A}_{N, p}$ равновероятны по мере $\mu_{N}$, что сводит задачу к классической теории случайных графов, см. [20]. Комбинаторньй метод доказательства этой теоремы состоит из нескольких шагов.

1. Назовем графф занумерованным, если его вершины занумерованы. Пусть $L_{N}(p)$ - число занумерованных $p$-регулярных графов с $N$ вершинами. Это число равно (см. теорему II.16 в [20])

$$
L_{N}(p) \sim C(p) \frac{(p N-1)(p N-3) \cdots}{(p !)^{N}},
$$

где $C(p)$ - константа, ее явньй вид известен, но нам не нужен. Аналогично для фиксированных $p, k, d_{1}, \ldots, d_{k}$ обозначим $L_{N}\left(p ; d_{1}, \ldots, d_{k}\right)$ - число занумерованных графов с какими-то $N-k$ вершинами степени $р$ и $N-k$ другими вершинами степеней $d_{1}, \ldots, d_{k}$ соответственно. Оно равно (см. также теорему II.16 в [20])

$$
L_{N}\left(p ; d_{1}, \ldots, d_{k}\right) \sim C\left(p, k ; d_{1}, \ldots, d_{k}\right) \frac{(2 m-1)(2 m-3) \cdots}{(p !)^{N}},
$$

где $C\left(p, k ; d_{1}, \ldots, d_{k}\right)$ - константа и $2 m=p(N-k)+d_{1}+\cdots+d_{k}$.

2. Докажем, что вероятность того, что окрестность $O_{1}(v)$ некоторой вершины (например, вершины 1) не содержит циклов, стремится к нулю при $N \rightarrow \infty$. Число графов таких, что $O_{1}(v)$ не содержит циклов, равно $L_{N-1}\left(p ; d_{1}, \ldots, d_{p}\right)(N-1) \cdots(N-p)$ с $d_{1}=\cdots=d_{p}=p-1$. В то же время число графов таких, что $O_{1}(v)$ содержит один цикл, т.е. одно дополнительное ребро, скажем, между ребрами 1 и 2 , равно $L_{N-1}\left(p ; e_{1}, \ldots, e_{p}\right)(N-1) \cdots(N-p)$, где $d_{1}=d_{2}=p-2, d_{3}=\cdots=d_{p}=p-1$. Легко видеть, что

$$
\frac{L_{N-1}\left(p ; e_{1}, \ldots, e_{p}\right)}{L_{N-1}\left(p ; d_{1}, \ldots, d_{p}\right)} \rightarrow 0
$$

при $N \rightarrow \infty$. 
3. Отсюда следует, что среднее число вершин $v$ с окрестностями $O_{1}(v)$ без циклов равно $o(N)$. Но так как почти все $p$-регулярные графы не имеют автоморфизмов (теорема IX.7 в [20]), то этот же результат верен и для незанумерованных графов.

4. Аналогично рассматриваются окрестности большего радиуса.

Отметим однако следующее важное обстоятельство: неверно, что для заданного $N$ вероятность того, что граф̆ из $\mathscr{A}_{N, p}$ является деревом, близка к 1 . Более того, вероятность, что данньй граб̆ в $\mathscr{A}_{N, p}$ есть дерево, стремится к 0 при $N \rightarrow \infty$. Нетривиальная топология (наличие циклов) возникает на большей шкале: типичная длина цикла стремится к $\infty$ при $N \rightarrow \infty$, см. ниже.

ЗАмЕчАниЕ 4 . Если множество $S$ конечно, есть нетривиальный спин, причем взаимодействие тривиально, то в пределе будет независимое поле на $p$-регулярном дереве. Вопрос: что будет, если есть дополнительный потенциал взаимодействия, например, двухчастичньй и ближайших соседей?

4.4.2. Гиббсовские многообразия групп. Кроме деревьев, гиббсовским способом можно выделять другие математические объекты, которые не допускают локальной характеризации. Мы рассмотрим случаи, когда в результате предельного перехода с вероятностью 1 возникает алгебраический объект - група.

Рассмотрим класс $\mathscr{H}_{N, 2 p} 2 p$-регулярных графов с локальной структурой, где все отростки каждой вершины занумерованы символами $a_{1}, \ldots, a_{p}, a_{1}^{-1}, \ldots, a_{p}^{-1}$. При этом если одному концу ребра соответствует, скажем, $a_{i}$, то другому соответствует $a_{i}^{-1}$, и наоборот. Этот класс выделяется из класса $\mathscr{A}_{N}$ локальным потенциалом $\Phi$ на ребрах, равньм 0 , если отростки ребра несут символы $a_{i}, a_{i}^{-1}$, и $\infty$ в остальных случаях, а также суперлокальным потенциалом $\Phi_{1}$ на окрестностях радиуса 1 , равным нулю, лишь если вершина имеет степень $2 p$ и ее отростки занумерованы разными символами.

В частности, диаграммы Кэли счетных групп, см. [29], принадлежат к этому классу графов с локальной структурой. Граф̆ групшы с конечным числом образуюших $a_{1}, \ldots, a_{p}$ и конечным числом определяюших соотношений $\alpha_{1}=\cdots=\alpha_{n}=1$, где $\alpha_{1}, \ldots, \alpha_{n}$ - слова, выделяется следуюшим нелокальным условием: любой цикл в графе порождается $\alpha_{1}, \ldots, \alpha_{n}$, и наоборот (напомним, что любому пути на графе можно поставить в соответствие слово, т.е. элемент группы). Свободная некоммутативная группа соответствует случаю $n=0$, т.е. когда нет определяющих соотношений.

ТЕОРема 11. Предельная гиббсовская мера на $\mathscr{A}_{\infty}^{(0)}$ с потенциалом $\Phi+\Phi_{1}$ сосредоточена на дереве, соответствующем свободной группе с п образующими.

Мы не приводим здесь доказательства, однако заметим, что оно отличается от предыдушего для $p$-регулярных графов тем, что множество отростков разбивается на $2 p$ подмножеств $A\left(a_{i}\right), A\left(a_{i}^{-1}\right)$ и свобода соединения отростков ограничена только тем, что отростки $A\left(a_{i}\right)$ можно соединять только с отростками из $A\left(a_{i}^{-1}\right)$.

Этот результат, по-видимому, допускает следующее широкое обобщение. Рассмотрим множество $\mathscr{H}_{N, 2 p}\left(\alpha_{1}, \ldots, \alpha_{n}\right)$ графов, соответствуюших групшам из многообразия групп, определяемых соотношениями $\alpha_{1}=\cdots=\alpha_{n}=1$ (и возможно другими). Тогда предельная гиббсовская мера сосредоточена на свободной относительно этого многообразия группе (т.е. только с соотношениями $\alpha_{1}=\cdots=\alpha_{n}=1$ ), так как 
образование дополнительных коротких циклов маловероятно. Такие результаты могут играть роль и в физике, объясняя, как нелокальные объекты могут получаться с большой вероятностью из локальных условий.

4.5. Шкалы. Здесь мы кратко скажем о важном обобщении, которое позволяет вьйти за рамки термодинамического предельного перехода, ведушего к предельным гиббсовским семействам.

Пусть заданы гиббсовские семейства $\mu_{N}$ на множествах $\mathscr{B}_{N}$ конечных графов с локальной структурой, где $N$ - некоторьй параметр, такой как число вершин, радиус и т. д. Они индуцируют меры $\nu_{N}$ на соответствующих множествах $\mathscr{G}_{N}$ конечных графов. Пусть также задана неубьваюшая функция $f: \mathbb{Z}_{+} \rightarrow \mathbb{R}_{+}$. Определим макроразмерность случайного (в смысле мер $\nu_{N}$ ) конечного графа на шкале $f(N)$. Пусть $O_{f(N)}(v)$ - окрестность вершины $v$ радиуса $f(N)$. Положим

$$
D_{N}(f)=\left\langle\frac{1}{|V(G)|} \sum_{v \in V(G)} \frac{\ln O_{f(N)}(v)}{\ln f(N)}\right\rangle_{\nu_{N}} .
$$

Если сушествует предел $\lim _{N \rightarrow \infty} D_{N}(f)=D(f)$, то $D(f)$ назовем макроразмерностью на шкале $f$.

Можно определять соответствуюшие инварианты для счетного гиббсовского семейства, получаемые предельным переходом $N \rightarrow \infty$. См., например, определение макроразмерности счетного комплекса в [30]. Такое определение макроразмерности, однако, соответствует минимальной шкале $f$, где $f(N)$ растет как угодно медленно с ростом $N$.

Аналогично можно определять другие топологические характеристики на разных шкалах. Пусть $\mathscr{B}_{N}=\mathscr{A}_{N}$, т.е. $V(G)=N$. Например, пусть $h\left(O_{d}(v)\right)$ - число независимых циклов в $d$-окрестности вершины $v$. Экспонента для числа независимых циклов на шкале $f$ определяется как

$$
b(f)=\lim _{N \rightarrow \infty} \frac{1}{N}\left\langle\sum_{v} \frac{h\left(O_{f(N)}(v)\right)}{\left|O_{f(N)}(v)\right|}\right\rangle,
$$

если предел существует.

ПРЕДЛОЖЕНИЕ 4. В условиях теоремы 10 имеем: $b(f)=0$ для любой шкальи $f(N) \leqslant(1-\varepsilon) \log _{p-1} N$, әде $\varepsilon>0-$ произвольно, и $p / 2-1$ для любой шкаль $f(N) \geqslant(1+\varepsilon) \log _{p-1} N$.

ДокАЗАтельство. Рассмотрим окрестность $O_{(1-\varepsilon) \log _{p-1} N}(v)$ некоторой вершины $v$. В ней может быть не более $(p-1)^{(1-\varepsilon) \log _{p-1} N}=N^{1-\varepsilon}$ вершин. Каждое из ребер, выходящих из этих вершин, инцидентно другой вершине этой окрестности с вероятностью не более $N^{-\varepsilon}$. Поэтому число таких ребер не превосходит $N^{-\varepsilon} p N^{1-\varepsilon}=$ $p N^{1-2 \varepsilon}$. С каждым же таким ребром возникает не более одного нового цикла. Отсюда и следует первое утверждение.

Для доказательства второго обозначим диаметр случайного графа через $D(N)$. Известно [20], что для любого $\varepsilon>0$

$$
P_{N}\left(1-\varepsilon<\frac{D(N)}{\log _{p-1} N}<1+\varepsilon\right) \rightarrow 0, \quad N \rightarrow \infty .
$$


Во всем графе число независимых циклов равно $(p / 2-1) N-1$, что вытекает из формулы Эйлера $V-L+M=1$, где $M$ - число линейно независимых циклов. Поэтому $M=L-V=(p N / 2)-N$.

4.6. О дискретной квантовой гравитации. В заключение весьма уместно сказать о развитии квантовой гравитации в настоящее время. Ответ на вопрос "Что такое квантовая гравитация?" не может быть точно сформулирован. Есть много подходов к формулировке и решению этой проблемы (безудержный оптимизм некоторых физических работ всегда быстро сменялся более спокойным взглядом на ситуацию). Как мы увидим сейчас, многие подходы укладываются в схему, разработанную в данной статье. Основная трудность, не говоря о необходимости фундаментальных идей, состоит в конкретизации модели, исходя из многочисленных требований физики, которые также все еше неясны.

В физике считается, что так называемая стандартная модель квантовой теории поля отвечает практическим нуждам, описьвая все (электромагнитное, слабое и сильное) взаимодействия в природе, кроме гравитационного. Хотя стандартная модель в размерности 4 пространства-времени еще не построена в смысле конструктивной квантовой теории поля (т.е. с точки зрения общепринятого взгляда на математическую строгость), она допускает по крайней мере четко формулируемые определения и утверждения-гипотезы. Последние могут быть даны как в рамках аксиом Уайтмана, так и в виде скейлинг-предела решетчатых приближений. Однако при всех попытках создания единой теории, включающей гравитационное взаимодействие, даже точные определения исчезают. То, что есть в настоящее время, - это куски "будущей теории”, основанные на разных физических, методологических и даже философских принципах. Я попытаюсь сделать некоторую их классификацию, оставаясь в рамках математики, т.е. точных определений и формулировок.

Заметим сначала, что не видно никаких способов прямого обобщения аксиом Уайтмана, или, в евклидовом варианте, Остервальдера-Шредера (хотя аксиомы типа $S$-матрицы, где пространство-время играет малую роль, обобщаются разными способами - аксиомы топологической теории поля, диаграммы теории струн). Все основные их понятия основаны на классическом и фиксированном пространстве-времени. Например, локальность использует метрику Минковского, а унитарность - само сушествование классического времени. Если, в рамках аксиом Уайтмана, присоединить к полям материи метрику как новое поле, то она (как показьвает опыт конструктивной теории поля) не будет непрерывной функцией, что может изменить топологию пространства-времени в малом, делая неясным само понятие локальности. Тогда говорят, что квантование метрики приводит к “квантованию” пространства-времени.

Решетчатое приближение представляет собой гораздо более благоприятный объект для обобщения. И действительно, все сушествующие теории допускают дискретную переформулировку. Так, обычная евклидова квантовая теория поля (ограничимся для простоты бозонными полями) имеет дело с решеткой $\mathbb{Z}_{\varepsilon}^{d}$ с шагом $\varepsilon$, объемом $\Lambda=[-N, N]^{d} \subset \mathbb{R}^{d}$, с полями $\phi(x) \in \mathbb{R}^{m}, x \in \mathbb{Z}_{\varepsilon}^{d}$, и со статсуммой

$$
Z_{\Lambda, \varepsilon}=\left[\prod_{x \in \Lambda \cap \mathbb{Z}_{\varepsilon}^{d}} \int_{\mathbb{R}^{m}} d \phi(x)\right] \exp \left(-\sum_{\left\langle x, x^{\prime}\right\rangle} \Phi\left(\phi(x), \phi\left(x^{\prime}\right) ; \varepsilon\right)\right),
$$


где $x, x^{\prime}$ - любая пара ближайших соседей в $\Lambda \cap \mathbb{Z}_{\varepsilon}^{d}, \Phi$ - вешественная функщия на $\mathbb{R}^{m} \times \mathbb{R}^{m}$, зависящая также от параметра $\varepsilon$. Основная математическая проблема существование пределов разнообразных величин при $\varepsilon \rightarrow 0$.

В рамках этой статьи мы можем лишь кратко перечислить разные направления в построении дискретных моделей. Мы покажем, что они являются гиббсовскими семействами, явно указав класс графов с локальной структурой и потенциал. Физические обзоры различных направлений в квантовой гравитации см. [31]-[33].

Важно отметить, что почти все перечисляемые ниже теории занимаются квантованием метрики, но не топологии, так как рассматриваемый класс граффов связан с единственньм фиксированным многообразием или с классом многообразий довольно ограниченной топологии. Ввиду последнего, а также того, что часть теории представляется единой для всех моделей, общая теория гиббсовских семейств представляется необходимой.

Теория Редже. Теория Редже - хронологически самый ранний (см. [34]) дискретный вариант классической общей теории относительности. В отличие от модели динамических триангулящий, где длины всех ребер считаются равными, в теории Редже они являются произвольными. Пусть фиксирована триангулящия $T$ гладкого многообразия $M$ размерности $d$, и пусть $S_{k}(T), k=0, \ldots, d,-$ множество $k$-мерных симплексов триангуляции $T$. Пусть $l_{i}$ - длина стороны $i \in S_{1}(T)$ и $\mathscr{L}(T)=\left\{l_{i}, i \in S_{1}(T)\right\}$ - множество наборов длин, по которьм на каждом симплексе восстанавливается положительно определенная евклидова метрика. Тогда статсумма имеет вид

$Z_{N}=\sum_{T:|T|=N} \exp (-S(T)) d \Lambda(T), \quad S(T)=\lambda \sum_{B \in S_{d-2}(T)} v(B) \varepsilon(B)+\mu \sum_{\Delta \in S_{d}(T)} v(\Delta)$,

где $d \Lambda(T)$ - ограничение меры $\prod_{i \in S_{1}(T)} d l_{i}$ на $\mathscr{L}(T), v(\cdot)$ означает объем, $\varepsilon(B)$ - дефектный угол $(d-2)$-мерной грани $B$, которьй мы не будем определять (см. [34]). Они могут быть выражены через $l_{i}$. Таким образом, в квантовом евклидовом подходе $l_{i}$ являются случайными величинами, удовлетворяюшими соответствуюшим неравенствам, которые усложняют введение свободной меры.

Динамические триангуляции. Пожалуй, наиболее богата результатами модель, назьваемая “динамические триангуляции”, возникшая из теории струн.

Заметим, что из всех направлений в квантовой гравитации только теория струн включает в себя стандартную модель и пытается доводить теорию до “числа". Хотя теория струн следует формальной технике квантовой теории поля, ни о каких аксиомах речи не идет. Теория струн развивает скорее теорию матрицы рассеяния, чем наши фундаментальные представления о пространстве-времени.

Дискретная струна в евклидовом подходе есть гиббсовское семейство на некоторых подмножествах $\mathscr{A}_{N}$ множества $\bigcup_{\rho, k} \mathscr{A}_{N}(\rho, k)$ триангулящий $T$ поверхностей рода $\rho$ с $k$ дырками. На каждом треугольнике $i$ определен спин $\sigma_{i}$ со значениями в $\mathbb{R}^{d}$. Обьчно в качестве $\mathscr{A}_{N}$ берется $\mathscr{A}_{N}=\mathscr{A}_{N}(0,0)$ или $\bigcup_{\rho} \mathscr{A}_{N}(\rho, 0)$. При этом каноническая статсумма имеет вид

$$
Z_{N}=\sum_{T} \int_{\mathscr{A}_{N}} \exp \left(-\lambda \rho(T)-\sum_{\langle i, j\rangle}\left(\sigma_{i}-\sigma_{j}\right)^{2}\right) \prod_{i \in T} d \sigma_{i}
$$


Другие подходы к теории струн имеют, однако, большую популярность. Например, спектр свободной бозонной струны может быть найден явно в гамильтоновом подходе, что не сделано (и, по-видимому, трудно сделать) в подходе динамических триангуляций. Это загадочное обстоятельство показьвает тонкость связей между разными подходами к одному и тому же физическому объекту.

Матричные теории. Матричные модели используются для перечисления карт (и более сложных объектов) на поверхностях. Гладкой картой называется тройка $(S, G, \phi)$, где $S$-гладкая компактная ориентированная поверхность, $G$ - связньй граф (одномерньй комплекс), $\phi: G \rightarrow S$ - вложение такое, что образы ребер граф̆а $G$ являются гладкими дугами, а дополнение состоит из областей, гомеоморфных дискам. Карта (или комбинаторная карта) есть класс эквивалентности гладких карт. При этом две гладкие карты $\left(S, G, \phi_{1}\right),\left(S, G, \phi_{2}\right)$ называются эквивалентньми, если существует гомеоморфизм $f: S \rightarrow S$, переводящий вершины и дуги $\phi_{1}(G)$ соответственно в вершины и дуги $\phi_{2}(G)$.

Модель случайных матриц есть вероятностное распределение $\mu$ на множестве самосопряженных $n \times n$-матриц $\phi=\left(\phi_{i j}\right)$ с плотностью

$$
\frac{d \mu}{d \nu}=Z^{-1} \exp \left(-\operatorname{tr}\left(\frac{\phi^{2}}{2 h}\right)-\operatorname{tr}(V)\right)
$$

где $V=\sum a_{k} \phi^{k}$ - полином от $\phi$, ограниченньй снизу, $\nu$ - мера Лебега на вешественном $n^{2}$-мерном пространстве векторов $\left(\phi_{i i}, \operatorname{Re} \phi_{i j}, \operatorname{Im} \phi_{i j}, i<j\right)$. Если $V=0$, то мера $\mu=\mu_{0}$ будет гауссовой с ковариациями $\left\langle\phi_{i j}, \phi_{k l}^{*}\right\rangle=\left\langle\phi_{i j}, \phi_{l k}\right\rangle=h \delta_{i k} \delta_{j l}$. Тогда плотность $\mu$ относительно гауссовой меры $\mu_{0}$ равна

$$
\frac{d \mu}{d \mu_{0}}=Z_{0}^{-1} \exp (-\operatorname{tr}(V))
$$

Для сушествования меры $\mu$ необходимо, чтобы старший коэффициент $a_{p}$ полинома $V$ был положительным, а $p$ было четным. Для этого случая сушествует хорошо развитая теория таких моделей, см. [35].

Фундаментальная связь (идушая от Хооф̆та) между матричными моделями и перечислением комбинаторных карт на поверхностях дается формальным рядом по семиинвариантам или по диаграммам (см. [36])

$$
\log Z=\sum_{k=1}^{\infty} \frac{(-1)^{k}}{k !}\langle\operatorname{tr}(V), \ldots, \operatorname{tr}(V)\rangle=\sum_{k=1}^{\infty} \frac{(-1)^{k}}{k !} \sum_{D_{k}} I\left(D_{k}\right),
$$

где $\sum_{D_{k}}$ - сумма по всем связным диаграммам $D_{k}$ с $k$ вершинами и $L=L\left(D_{k}\right)$ ребрами. Пусть, например, $V=a \phi^{4}$. Тогда каждая диаграмма имеет занумерованные вершины $1, \ldots, k, L=2 k$ ребер, каждая вершина имеет занумерованные отростки $1,2,3,4$, соответству юшие множителям произведения $\phi_{i j} \phi_{j k} \phi_{k l} \phi_{l i}$. Отросток, например. соответствуюший $\phi_{i j}$, можно представлять как двустороннюю полоску (определяя так назьваемый “ленточный”, или “толстый”, граф), стороны которой имеют матричные индексы $i$ и $j$ соответственно. При этом в окрестности вершины полоски 


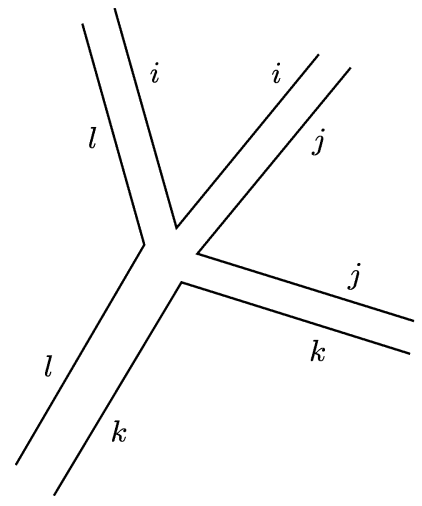

Рис. 2. Вершина ленточного графа

укладьваются на плоскости согласно приводимому рисунку. Договоримся, что спаривание отростков-полосок делается так, что спаренные стороны имеют одинаковые индексы.

Так как индекс встречается четное число раз в каждой вершине, то для данного ребра $l$ с индексом $i$ есть единственный связный путь в диаграмме по ребрам с индек$\operatorname{coм} i$, проходящий через $l$. Эти пути замкнуты и назьваются индексными петлями. Суммируя тогда по индексам, мы получим множитель $n^{N}$, где $N=N\left(D_{k}\right)$ - число индексных петель. В результате мы получим формальньй ряд

$$
\sum_{k} \frac{(-a)^{k}}{k !} \sum_{D_{k}} h^{2 k} n^{N\left(D_{k}\right)}=\sum_{k}\left(-4 a h^{2}\right)^{k} \sum_{E_{k}} n^{N\left(E_{k}\right)}=\sum_{k, N}\left(-4 a h^{2}\right)^{k} n^{N} M(k, N),
$$

где в сумме по $E_{k}$ суммирование ведется по всем графам с неупорядоченным множеством вершин, а множество отростков каждой вершины циклически упорядочено. При переходе от $D$ к $E$ введен множитель $\frac{k ! 4^{k}}{A(E)}$, где $A(E)$ - мошность группы автоморфизмов $E$. Мы опускаем $A(E)$, считая, что для "почти всех" (при $k \rightarrow \infty$ ) графов $A(E)=1$.

В последней сумме $M(k, N)$ - число карт с $k$ вершинами и $N$ клетками. Действительно, нетрудно понять, что для каждого граффа $E$ существует единственное (с точностью до комбинаторной эквивалентности) вложение $f(E)$ этого графа в компактную ориентируемую поверхность $S_{\rho}$ некоторого рода $\rho$ такое, что каждая индексная петля ограничивает открытую область поверхности $S_{\rho}$, гомеоморфную диску. Эта карта имеет $k$ вершин, $2 k$ ребер и $N$ граней. По формуле Эйлера $k=N+2 \rho-2$ получаем следуюшее разложение, полагая $h=1, a=b / n$ :

$$
\log Z=\sum_{N, \rho}(-4 b)^{N+2 \rho-2} n^{-2 \rho+2} M_{\rho}(N)
$$

где $M_{\rho}(N)$ - число карт рода $\rho$ с $N$ клетками. Отсюда, например, при $\rho=0$ члены $M_{\rho}(N)$ выделяются формально как

$$
\lim _{n \rightarrow \infty} \frac{\log Z}{n^{2}}=\sum_{N}(-4 b)^{N-2} M_{0}(N) .
$$


Вообще следует сказать, что применение матричного метода к комбинаторным задачам является строгим только для подсчета числа триангулящий поверхности с одной (см. [37]) или конечным числом клеток (см. [38], [39]). Очень ясно написанное введение в матричный метод для карт для математика можно найти в [40], полные, но менее математичные изложения имеются во многих физических работах, см. [41]. Случай фиксированного рода $\rho$ (который соответствует подсчетам диаграмм в теории струн по теории возмущений) хорошо продвинут только в случае размерности $d=0$ вмешаюшего пространства или, на физическом уровне строгости, для следующего обобщения - для модели с $Q$ матрицами $M_{q}$, где действие имеет вид

$$
\operatorname{Tr}\left(\sum_{q=1}^{Q} V\left(M_{q}\right)+\sum_{q=1}^{Q-1} M_{q} M_{q+1}\right)
$$

а также для его непрерьвного аналога, см. обзор [42].

Необходимо отметить, что случай больших (т.е. растуших вместе с $N$ ) $\rho$, соответствующий на физическом языке изучению "пены” пространства-времени в смысле Хокинга, почти не изучен даже при отсутствии спина, т.е. для $d=0$.

Спиновая пена. Спиновой пеной назьвается граф̆ Г вместе с локальной структурой двумерного комплекса (при этом каждая вершина и каждое ребро принадлежат по крайней мере одной двумерной клетке) и функциями $a(f)$ на множестве $\Gamma^{(2)}$ двумерных клеток $f$ и $b(l)$ на множестве ребер $\Gamma^{(1)}$. При этом $a(f)$ задает неприводимое представление групшы (Ли или квантовой) $G$, пусть $H_{a(f)}$ - гильбертово пространство этого представления. Если ребро $l$ инцидентно клеткам $f_{1}, \ldots, f_{k}$, то положим $H_{l}=H_{a\left(f_{1}\right)} \otimes \cdots \otimes H_{a\left(f_{k}\right)}$. Пусть $H_{l}^{0}$ - инвариантное подпространство в $H_{l}$. Выберем раз и навсегда в инвариантных пространствах тензорного произведения произвольных неприводимых представлений ортонормированные базисы. Тогда функция $b(l)$ задает один из элементов этого базиса.

На некотором таком классе конечных спиновых графов $\mathscr{A}$ (например, с $N$ вершинами) со структурой двумерного комплекса определим статистическую сумму

$$
Z_{\mathscr{A}}=\sum_{\Gamma \in \mathscr{A}} Z(\Gamma), \quad Z(\Gamma)=\sum_{\{a(\cdot), b(\cdot)\}} \prod_{f \in \Gamma^{(2)}} \operatorname{dim} a(f) \prod_{v \in \Gamma^{(0)}} \Phi\left(O_{1}(v)\right)
$$

где $\Phi$ - некоторая функция от окрестности вершины $v$ радиуса 1 . Если $\Phi$ положительна, то этим задается гиббсовское семейство.

Насколько мне известно, такие статсуммы рассматривались только как формально алгебраические объекты. Вообше, в физике часто пишутся статсуммы, не являющиеся положительными, и вопрос о придании смысла величинам в этом случае полностью открыт. Квантовые дискретные пространства, вводимые в следующем ниже разделе 5, могут быть полезнее гиббсовских.

Приведем несколько примеров, подробнее см. [43]. Для вершины $v$ обозначим $H_{v}=$ $H_{l_{1}} \otimes \cdots \otimes H_{l_{m}}$, где $l_{1}, \ldots, l_{m}$ - все 1-клетки, инцидентные $v$. Так как каждая 2-клетка $f_{i}$ инцидентна ровно двум ребрам, исходящим из $v$, то соответствующее $H_{a\left(f_{i}\right)}$ появляется в $H_{v}$ дважды. Поэтому $H_{v}$ естественно представляется как тензорный квадрат, и 
для каждого его элемента определен след Tr. Тогда модель Тураева-Огури-КрейнаЙеттера задается функцией

$$
\Phi=\operatorname{Tr}\left(b\left(l_{1}\right) \otimes \cdots \otimes b\left(l_{m}\right)\right) .
$$

Эта статсумма конечна, если в качестве группы рассматривается квантовая группа $S U(2)_{q}$. Основной результат состоит в том, что $Z(\Gamma)$ не зависит от $\Gamma$, если $Г$ пробегает двумерный остов триангулящии данного 4-мерного многообразия. 3-мерньй вариант этой модели - модель Тураева-Виро, а 3-мерньй вариант с группой $S U(2)$ - модель Понцано-Редже (см. [44]), развиваюшая модель Редже и постулируюшая дискретность длин ребер: $l=\delta j, j=0, \frac{1}{2}, 1, \frac{3}{2}, \ldots$ С каждым $j$ связьвается представление групшы $S U(2)$ спина $j$.

Модели спиновой пены дают возможность гиббсовского подхода к так назьваемой петлевой квантовая гравитации, которая ранее рассматривалась в рамках гамильтонова подхода. Она основывалась на новых переменных, введенных Аштекаром в общей теории относительности, и использовала сети Пенроуза как удобньй базис в пространстве функций на множестве всех связностей в пространстве в фиксированный момент времени.

Топологическая квантовая теория поля. Аксиомы топологической квантовой теории поля в [23] скорее напоминают аксиомы $S$-матрицы, нежели дают модели структуры пространства-времени. Гиббсовские семейства ближе физическому представлению топологической теории поля в терминах функциональных интегралов. Известные ее модели имеют дело с конечномерньми гильбертовыми пространствами, отнесенными граничным многообразиям. Классификация таких случаев в размерности 2 имеется в последней главе в [27]. Здесь мы можем только сказать о соответствующих проблемах с точки зрения гиббсовских семейств.

Рассмотрим триангуляии двумерной компактной ориентируемой поверхности рода $\rho$ с $k$ дырками, пусть $S$ - спиновое пространство. Рассмотрим множество $\mathscr{T}\left(N, m_{1}, \ldots, m_{k}\right)$ триангулящй с заданным числом $N$ треугольников и заданными $m_{1}, \ldots, m_{k}$ - числами ребер на каждой из $k$ границ. Для заданного потенциала можно рассматривать условную статсумму $Z_{m_{1} \ldots m_{k}}\left(s_{\text {boundary }}\right)$ при заданных значениях $s_{\text {boundary }}$ спина на гранище, что дает положительную (ненормированную) меру $\mu\left(m_{1}, \ldots, m_{k}\right)$ на конфигурациях $S^{m_{1}} \times \cdots \times S^{m_{k}}$ и меру на

$$
D(k)=\bigcup_{m_{1}, \ldots, m_{k}} S^{m_{1}} \times \cdots \times S^{m_{k}}
$$

для данного $k$, или даже на $\bigcup_{k} D(k)$. В частности, когда эти меры конечны и при каких скейлингах возможен слабьй предел этих мер? Это может дать примеры, когда гильбертовы пространства имеют бесконечную размерность.

Причинные множества. Причинным множеством $V$ (см. краткий обзор [45]) называется частично-упорядоченное множество с отношением $\leqslant$, удовлетворяюшее условиям транзитивности, рефлексивности и ащикличности (если $x \leqslant y$ и $y \leqslant x$, то $x=y$ ). Данное (конечное или счетное) причинное множество определяет направленньй граф $G$ (диаграмма Хассе $V$ ), так что $V$ становится множеством вершин $G$, причем от $x$ к $y$ есть направленное ребро, если $x \leqslant y, x \neq y$, и если нет другого $z$ такого, 
что $x \leqslant z \leqslant y$. Наоборот, любой направленный граф без циклов определяет причинное множество $V$. Поэтому причинные множества сводятся к графам с локальной структурой. Причинные множества интересны тем, что для них могут быть определены дискретные аналоги светового конуса и других используемых в общей теории относительности понятий.

Некоммутативные решетки. С некоммутативной $C^{*}$-алгеброй можно связать двойственньй объект, интерпретируемьй как дискретное некоммутативное пространство. Эта тема относится уже к следуюшей (квантовой) части статьи, и мы скажем только несколько слов.

Топологические пространство $X$ можно аппроксимировать дискретньми множествами двумя способами: либо брать все более плотные его подмножества, либо с помощью вложенной последовательности покрытий $\mathscr{U}^{n}=\left\{\mathscr{U}_{i}^{n}\right\}$ (как множество всех замкнутых точек их проективного предела). Произвольное покрытие компактного пространства $X$ порождает конечное разбиение $X$, а множество блоков этого разбиения будет $T_{0}$-пространством в фактор-топологии. Задание $T_{0}$-топологии на конечном или счетном множестве эквивалентно заданию отношения частичного порядка на нем: $x \leqslant y$ тогда и только тогда, когда $y$ принадлежит замьканию $\{x\}$. Как мы видели, с частично упорядоченным множеством естественно связьвается направленный граф̆его диаграмма Хассе.

Каждое конечное частично упорядоченное множество $V$ является множеством неприводимых представлений $\widehat{A}$ некоторой (вообще говоря, не единственной) $C^{*}$-алгебры $A$, см. [46]. Если, кроме того, $A$ сепарабельна, то множество $\widehat{A}$ гомеоморфино пространству $\operatorname{prim} A$ примитивных идеалов $A$, которое назьвается некоммутативной решеткой. Применение некоммутативных решеток в простой квантовомеханической задаче см. в последней главе [46].

\section{5. Квантовые дискретные пространства}

5.1. Квантовые графы. Пусть сначала $S$ не более чем счетно. Обозначим $\mathscr{H}=$ $l_{2}\left(\mathscr{A}^{(0)}\right)$ - гильбертово пространство с ортонормированным базисом $e_{\alpha}$, занумерованным конечными спинграффами $\alpha \in \mathscr{A}^{(0)}:\left(e_{\alpha}, e_{\beta}\right)=\delta_{\alpha \beta}$, где функция $e_{\alpha}(\beta)=\delta_{\alpha \beta}$. Каждый вектор $\phi$ из $\mathscr{H}$ является функцией на множестве $\mathscr{A}^{(0)}$ и может быть записан каK

$$
\phi=\sum \phi(\alpha) e_{\alpha} \in \mathscr{H}, \quad\|\phi\|^{2}=\sum|\phi(\alpha)|^{2}
$$

Состояния системы - волновые функции, т.е. векторы $\phi$ с единичной нормой $\|\phi\|^{2}=$ $\sum|\phi(\alpha)|^{2}=1$.

Определение квантовых “гиббсовских" состояний отличается от классических тем, что в квантовом случае автоматически получается динамика, или гамильтониан. Динамика на квантовых дискретных пространствах связана с подстановками. Грубо говоря, подстановка состоит в замене одного регулярного подграфа $\gamma$ другим $\delta$, но точное определение сложнее, так как надо позаботиться о правиле соединения $\delta$ с дополнением к $\gamma$.

Вершину $v \in V(\gamma) \subset V(G)$ регулярного подграфа $\gamma$ графа $G$ назовем внутренней, если любое инщидентное ей ребро соединяет ее с другой вершиной $\gamma$, и назовем ее гра- 
ничной в противном случае. Множество всех граничных вершин назовем границей $\partial \gamma$ регулярного подграфа $\gamma$.

ОПРЕДЕЛЕНИЕ 5. Правило подстановки (продукция) $\mathrm{Sub}=\left(\Gamma, \Gamma^{\prime}, V_{0}, V_{0}^{\prime}, \varphi\right)$ определяется двумя “мальми” спиновыми графами $\Gamma$ и $\Gamma^{\prime}$, подмножествами $V_{0} \subset V=$ $V(\Gamma), V_{0}^{\prime} \subset V^{\prime}=V\left(\Gamma^{\prime}\right)$ и взаимно однозначным отображением $\varphi: V_{0} \rightarrow V_{0}^{\prime}$, сохраняюшим спины. Пусть имеется изоморфизм $\psi: \Gamma \rightarrow \gamma$ на регулярныи спиновьй подграфф $\gamma$ спинграфа $\alpha$ такой, что $\psi\left(V_{0}\right) \supset \partial \gamma$. Преобразование (подстановка) $T=T(\mathrm{Sub}, \psi)$ спинграфа $\alpha$, соответствуюшее данному правилу подстановки Sub и изоморфизму $\psi$, определяется следуюшим образом. Удалим все ребра подграфа $\gamma=\psi(\Gamma)$, удалим все вершины $V(\gamma) \backslash \psi\left(V_{0}\right)$, в несвязном объединении $\alpha$ и $\Gamma^{\prime}$ отождествим каждое $\psi(v)$, $v \in V_{0}$, с $\varphi(v) \in V_{0}^{\prime}$. Обозначим получившийся спинграф̆ $T(\mathrm{Sub}, \psi) \alpha$. Функция $s$ на $V(\alpha) \backslash V(\gamma)$ наследуется из $\alpha$, а на $V(\gamma)-$ из $\Gamma^{\prime}$.

Примеры подстановок: удаление ребра, добавление ребра в данной вершине с другой новой вершиной, соединение двух вершин ребром, изменение значения спина в одной вершине. При этом сама возможность такой подстановки зависит от некоторой окрестности места подстановки.

Для произвольных $N$ и $p$ пусть $\mathscr{H}_{N, p} \subset \mathscr{H}$ - конечномерное подпространство, порожденное всеми $e_{\alpha}, \alpha \in \bigcup_{n=0}^{N} \mathscr{A}_{n, p}^{(0)}$, пусть $P_{N}$ - ортогональньй проектор на $\mathscr{H}_{N, p}$. Для данного $p$ имеются естественные вложения $\mathscr{H}_{N, p} \subset \mathscr{H}_{N+1, p}$. Далее $p$ фиксировано и этот индекс мы будем опускать, имея в виду, что степени вершин не превосходят $p$. Мь будем обозначать $\mathscr{H}_{N}=\mathscr{H}_{N, p}$.

ОПредЕлЕниЕ 6. Грамматика (точнее, грамматика на графах) определяется конечньм множеством подстановок $\mathrm{Sub}_{i}=\left(\Gamma_{i}, \Gamma_{i}^{\prime}, V_{i, 0}, V_{i, 0}^{\prime}, \varphi_{i}\right), i=1, \ldots,|\mathrm{Sub}|$. Она называется локальной, если для всех $i$ графы $\Gamma_{i}$, соответствующие $\mathrm{Sub}_{i}$, связны. Будем назьвать грамматику локально ограниченной, если при достаточно больших $p$ множества $\bigcup_{N} \mathscr{A}_{N, p}^{(0)}$ инвариантны относительно всех ее подстановок.

Определим основные операторы $a_{i}(j)$. Индекс $i$ соответствует одной из подстановок грамматики. Для данного $i$ рассмотрим всевозможные пары $(\psi, \xi)$, где $\xi \in \mathscr{A}^{(0)}$, а $\psi$ - отображение $\Gamma_{i}$ на регулярный подграф̆ спинграффа $\xi$. При этом пара $(\psi, \xi)$ называется минимальной, если $\psi\left(\Gamma_{i}\right)$ не принадлежит окрестности 0 радиуса меньшего, чем радиус $R(\xi)=R_{0}(\xi)$ самого графа $\xi$. Для данного $i$ занумеруем все регулярные пары индексами $j=1,2, \ldots$ :

$$
\left(\psi_{i 1}, \xi_{i 1}\right),\left(\psi_{i 2}, \xi_{i 2}\right), \ldots,\left(\psi_{i j}, \xi_{i j}\right), \ldots
$$

Если в $\xi$ есть два регулярных подграфа, изоморфных $\Gamma_{i}$ и переходяших друг в друга при нетривиальном автоморфизме граффа $\xi$, то им соответствуют разные индексы $j$ в нашей нумерации.

Положим

$$
a_{i}(j) e_{\alpha}=e_{\beta}, \quad \beta=T\left(\operatorname{Sub}_{i}, \rho \psi_{i j}\right) \alpha
$$

для любого $\alpha$ такого, что сушествует изоморфизм $\rho$ спинграфа $\xi_{i j}$ на окрестность $O_{R(\xi)}(0)$ начала координат графа $\alpha$. Положим $a_{i}(j) e_{\alpha}=0$ в остальных случаях. Ясно, что это определение не зависит от выбора $\rho$. Заметим, что $\left\|a_{i}(j)\right\|=1$. 
Определим линейньй оператор $H=H\left(\left\{\mathrm{Sub}_{i}, i=1, \ldots, k\right\}\right)$, соответствующий грамматике $\mathrm{Sub}_{i}=\left(\Gamma_{i}, \Gamma_{i}^{\prime}, V_{i, 0}, V_{i, 0}^{\prime}, \varphi_{i}\right), i=1, \ldots, k$, как формальную сумму

$$
H=\sum_{i=1}^{k} \sum_{j} \lambda_{i} a_{i}(j)
$$

для некоторых комплексных констант $\lambda_{i}$. Тогда $H$ определен на линейном пространстве $\mathscr{H}^{0}$ конечных линейных комбинаций $e_{\alpha}$. Важно отметить, что этот оператор не зависит от нумерации $\psi_{i, j}$ ввиду следующего эквивалентного определения: для всех $\alpha$

$$
H e_{\alpha}=\sum_{i=1}^{k} \sum_{\psi} e_{T\left(\mathrm{Sub}_{i}, \psi\right) \alpha}
$$

где для данного $i$ сумма берется по всем вложениям $\psi: \Gamma_{i} \rightarrow \alpha$.

Сопряженная подстановка $\mathrm{Sub}^{*}=\left(\Gamma^{*}, \Gamma^{\prime *}, V_{0}^{*}, V_{0}^{\prime *}, \varphi^{*}\right)$ к подстановке Sub определяется свойствами: $\Gamma^{*}=\Gamma^{\prime}, \Gamma^{*}=\Gamma, V_{0}^{*}=V_{0}^{\prime}, V_{0}^{\prime *}=V_{0}, \varphi^{*}=\varphi^{-1}$. Если подстановки $i$ и $i_{1}$ сопряжены, то для любого $j$ сушествует такое $j_{1}$, что $\left(a_{i}(j)\right)^{*}=a_{i_{1}}\left(j_{1}\right)$.

Гамильтониан формально симметричен, $H=H^{*}$, в случае, если для каждого $i$ сушествует $j$ такое, что $\mathrm{Sub}_{j}$ является сопряженной к $\mathrm{Sub}_{i}$ и $\lambda_{i}=\bar{\lambda}_{j}$.

Одномерные грамматики. Если $p=2$, то граф̆ линеен, цикличен или нет. Эквивалентно можно говорить о слове $\alpha=x_{1} \ldots x_{n}, x_{i} \in S$. В этом случае правило подстановки есть пара $\gamma \rightarrow \delta$, а подстановка состоит в преобразовании

$$
\alpha \gamma \beta \rightarrow \alpha \delta \beta
$$

для любых $\alpha, \beta$, где конкатенация слов $\alpha=x_{1} \ldots x_{n}$ и $\beta=y_{1} \ldots y_{m}$ определяется как

$$
\alpha \beta=x_{1} \ldots x_{n} y_{1} \ldots y_{m}
$$

Оператор $a_{i}(j)$ разрешает делать подстановку $i$ в слове $\alpha$ только для подслова $\gamma_{i}$, начинаюшегося с символа с номером $j$. Сопряженной к подстановке $\gamma \rightarrow \delta$ является подстановка $\delta \rightarrow \gamma$.

Самосопряженность. Пусть $H$ симметричен на линейном пространстве $\mathscr{H}^{0}$ конечных линейных комбинаций $e_{\alpha}$. Они являются $C^{\infty}$-векторами (см. [47]) для $H$, т.е. $H e_{\alpha} \in \mathscr{H}^{0}$. Заметим, что $H$, вообще говоря, неограничен.

ТеОРема 12. Пусть грамматика локально ограничена и Н симметричен. Тогда Н существенно самосопряжен на $\mathscr{H}^{0}$.

Доказательство следует из следуюшей леммы и критерия Нельсона, см. [47]. Это определяет группу $\exp (i t H)$. В лемме не требуется симметричности гамильтониана. 
ЛЕмма 10. Для любой грамматики произвольный вектор $\phi \in \mathscr{H}^{0}$ является аналитическим для соответствующего гамильтониана, т.е.

$$
\sum_{k=0}^{\infty} \frac{\left\|H^{k} \phi\right\|}{k !} t^{k}<\infty
$$

для некоторого $t>0$.

Достаточно взять $\phi=e_{\alpha}$ для некоторого $\alpha$. В этом случае число пар $(i, j)$ таких, что $a_{i}(j) e_{\alpha} \neq 0$, не превосходит $C V, V=V(\alpha)$, для некоторой константы $C$. Действительно, число регулярных подграфов, изоморфных данному "малому" подграфу, зависит от числа вершин и от $p$. Напишем разложение $H$ в виде

$$
H=\sum_{a} V_{a}
$$

где $V_{a}$ равен одному из $\lambda_{i} a_{i}(j)$. Тогда

$$
H^{n} e_{\alpha}=\sum_{a_{n}, \ldots, a_{1}} V_{a_{n}} \cdots V_{a_{1}} e_{\alpha}=\sum C_{\beta} e_{\beta}
$$

Максимальное число вершин графов $\beta$ в разложении $V_{a_{n}} \cdots V_{a_{1}} e_{\alpha}$ не превосходит $V(\alpha)+C_{1} n$, так как каждьй множитель прибавляет число вершин, не большее некоторой константы $C_{1}$. Тогда для данных $e_{\alpha}, a_{1}, \ldots, a_{n}$ число операторов $V_{a_{n+1}}$, даюших ненулевой вклад в $V_{a_{n+1}} V_{a_{n}} \cdots V_{a_{1}} e_{\alpha}$, не превосходит $C\left(V(\alpha)+C_{1} n\right)$ для некоторой константы $C$. Значит, число ненулевых членов $V_{a_{n}} \cdots V_{a_{1}} e_{\alpha}$ не превосходит

$$
\begin{aligned}
C^{n} \prod_{j=1}^{n}\left(|V(\alpha)|+C_{1} j\right) & =\left(C C_{1}\right)^{n} \prod_{j=1}^{n}\left(\frac{|V(\alpha)|}{C_{1}}+j\right) \\
& <\left(C C_{1}\right)^{n} n ! \prod_{j=1}^{n}\left(1+\frac{|V(\alpha)|}{C_{1} j}\right)<\left(C C_{1}\right)^{n} n ! n^{2 \frac{|V(\alpha)|}{C_{1}}}
\end{aligned}
$$

а норма каждого члена ограничена $\left(\max \lambda_{i}\right)^{n}$. Это и дает сходимость ряда для $|t|<t_{0}$, где $t_{0}$ не зависит от $\alpha$. Лемма доказана.

5.1.1. $C^{*}$-алгебры. Есть несколько полезных $C^{*}$-алгебр, связанных с грамматиками на графах: две универсальные $\mathbf{B}, \mathbf{C}$ и одна зависяшая от грамматики $\mathbf{A}(G r)$. Определим эти алгебры.

Пусть $\mathbf{C}_{N}$ - алгебра всех операторов в конечномерном пространстве $\mathscr{H}_{N}=\mathscr{H}_{N, p}$. Оператор $C \in \mathbf{C}_{N}$ можно считать действующим в $\mathscr{H}$, если положить $C e_{\alpha}=0$ для $e_{\alpha} \notin \mathscr{H}_{N, p}$. Тогда $\mathbf{C}_{N} \subset \mathbf{C}_{N+1}$, положим $\mathbf{C}=\overline{\bigcup_{N} \mathbf{C}_{N}}$. Алгебра $\mathbf{C}$ состоит из компактных операторов. Она использовалась в [48].

Пусть $\mathbf{A}_{N}=\mathbf{A}_{N}\left(\mathrm{Sub}_{i}, i=1, \ldots, k\right)-C^{*}$-алгебра операторов в $\mathscr{H}$, порожденная всеми $a_{i}(j)$ такими, что $R\left(\xi_{i j}\right) \leqslant N$. Тогда определены естественные вложения $\phi_{N}: \mathbf{A}_{N} \rightarrow \mathbf{A}_{N+1}$. Индуктивньй предел $\bigcup_{N} \mathbf{A}_{N}=\mathbf{A}^{0} C^{*}$-алгебр $\mathbf{A}_{N}$ назьвается локальной алгеброй, а его замыкание по норме $\mathbf{A}=\mathbf{A}\left(\mathrm{Sub}_{i}, i=1, \ldots, k\right)$ - квазилокальной алгеброй. Структура $C^{*}$-алгебры $\mathbf{A}=\mathbf{A}\left(\mathrm{Sub}_{i}, i=1, \ldots, k\right)$ зависит от 
грамматики и от фиксированного класса графов. Ниже мы приводим цельй ряд примеров гамильтонианов и $C^{*}$-алгебр.

Универсальная $C^{*}$-алгебра $\mathbf{B}$ порождена операторами $a_{i}(j)$ для всех возможных подстановок. В не совпадает с алгеброй всех ограниченных операторов - например, многие диагональные операторы в базисе $e_{\alpha}$ не включены в $\mathbf{B}$.

5.1.2. Автоморфизмы. Далее всегда предполагается локальная ограниченность грамматики и фиксируется $p$ такое, что множества $\bigcup_{N} \mathscr{A}_{N, p}^{(0)}$ инвариантны относительно всех ее подстановок.

Формальный гамильтониан определяет дифференщирование локальной алгебры $\mathbf{A}^{0}$. Рассмотрим

$$
H_{N}=\sum_{i=1}^{\mid \text {Sub } \mid} \sum_{j: R\left(\xi_{i j}\right) \leqslant N} \lambda_{i} a_{i}(j)
$$

Определим групшу автоморфизмов А следуюшим образом. Возьмем локальньй элемент $A \in \mathbf{A}^{0}$ и $N$ такое, что $A \in \mathbf{A}_{N}$, и положим

$$
\alpha_{t}^{(N)}(A)=\exp \left(i H_{N} t\right) A \exp \left(-i H_{N} t\right)
$$

Теорема 13. Существует $t_{0}>0$ такое, что для любого локального $A$ и любого $t,|t|<t_{0}$, существует предел по норме

$$
\lim _{N \rightarrow \infty} \alpha_{t}^{(N)}(A)
$$

Это определяет единственный автоморфизм квазилокальной алгебры.

ДокАЗАТЕльствО. Рассмотрим ряд Дайсона-Швингера

$$
A_{t}^{(N)}=A+\sum_{n=1}^{\infty} \frac{(i t)^{n}}{n !}\left[H_{N}, \ldots,\left[H_{N},\left[H_{N}, A\right]\right] \ldots\right] .
$$

Можно ограничиться локальньм $A=a_{i}(k)$ для некоторых $i, k$.

Лемма 11. Норма оператора $\frac{(i t)^{n}}{n !}\left[H_{N}, \ldots,\left[H_{N},\left[H_{N}, A\right]\right] \ldots\right]$ ограничена сверху посредством $(C t)^{n}$ независимо от $N$.

Заметим, что коммутатор принадлежит А и равен сумме коммутаторов вида

$$
\left[a_{i_{n}}\left(j_{n}\right), \ldots,\left[a_{i_{2}}\left(j_{2}\right),\left[a_{i_{1}}\left(j_{1}\right), A\right]\right] \ldots\right]
$$

умноженных на $\lambda_{i_{1}} \cdots \lambda_{i_{n}}$. Каждьй из коммутаторов равен сумме $2^{n}$ членов вида

$$
\pm a_{q_{n}}\left(p_{n}\right) \cdots a_{q 2}\left(p_{2}\right) a_{q_{1}}\left(p_{1}\right)
$$

Докажем, что после сокрашений останется не более $\prod_{j=1}^{n} C j=C^{n} n$ ! таких членов (независимо от $N$ ). Для этого надо воспользоваться вторым определением гамильтониана. Введем обозначение: если $a_{i}(j) e_{\alpha}=e_{T(\operatorname{Sub}, \psi)}$, то будем писать $a_{i}(j) e_{\alpha}=$ $a(T(\mathrm{Sub}, \psi)) e_{\alpha}$. Тогда для любого $\alpha$, любых двух правил подстановки $i_{1}, i_{2}$ и двух 
отображений $\psi_{1}: \Gamma_{i_{1}} \rightarrow \gamma_{1}, \psi_{2}: \Gamma_{i_{2}} \rightarrow \gamma_{2}$ на пересекающиеся регулярные подграфы $\gamma_{1}, \gamma_{2}$ графа $\alpha$ соответствуюшие преобразования в терминах $a_{i}(j)$ дадут сокрашаюшиеся члены:

$$
\begin{aligned}
& a\left(T\left(T\left(\mathrm{Sub}_{1}, \psi_{1}\right) \alpha, \mathrm{Sub}_{2}, \psi_{2}\right)\right) a\left(T\left(\alpha, \mathrm{Sub}_{1}, \psi_{1}\right)\right) e_{\alpha} \\
& \quad-a\left(T\left(T\left(\mathrm{Sub}_{2}, \psi_{2}\right) \alpha, \mathrm{Sub}_{1}, \psi_{1}\right)\right) a\left(T\left(\alpha, \mathrm{Sub}_{2}, \psi_{2}\right)\right) e_{\alpha}=0
\end{aligned}
$$

и т. д. по индукции.

Подобным образом можно доказать, при $N \rightarrow \infty A_{t}^{(N)}$ сходится почленно (для любого $n$ ) к ряду

$$
A_{t}=A+\sum_{n=1}^{\infty} \frac{(i t)^{n}}{n !}[H, \ldots,[H,[H, A]] \ldots] .
$$

Каждьй член последнего ряда определен, и ряд сходится, ввиду данной оценки, по норме для всех достаточно малых $t$. Сушествование группы автоморфизмов для всех $t$ может быть доказано теперь, как в теореме Робинсона для квантовых спиновых систем, см. [4].

5.1.3. КМШ-состояния на А. Здесь мы будем рассматривать КМШ-состояния на $\mathbf{A}$, которые являются естественньм обобщением КМШ-состояний на решетке. Иногда, однако, полезно рассматривать КМШ-состояния на алгебре $\mathbf{C}$ (см. [48]), где ситуация иная.

Мы ограничимся случаем $p=2$, т.е. одномерным линейным случаем, и будем предполагать, что $2 \leqslant|S|<\infty$. Алгебра $\mathbf{A}_{N}$, в отличие от квантовых спиновых систем, вообще говоря (если при подстановках меняется длина слова), не является конечномерной. Можно ввести конечномерные алгебры $\mathbf{F}_{N}$, порожденные всеми операторами $a_{i}^{(N)}(j)=P_{N} a_{i}(j) P_{N}$. Будем считать, что эти операторы действуют в $\mathscr{H}_{N}$. Определим состояния на $\mathbf{F}_{N}$, положив для любого $A_{N} \in \mathbf{F}_{N}$

$$
\begin{gathered}
\left\langle A_{N}\right\rangle_{N}=Z_{N}^{-1} \operatorname{Tr}_{N}\left[A_{N} \exp \left(-\beta P_{N} H P_{N}\right)\right] \\
Z_{N}=\operatorname{Tr}_{N} \exp \left(-\beta P_{N} H P_{N}\right)
\end{gathered}
$$

где $\operatorname{Tr}_{N}-$ след в $\mathscr{H}_{N}$. Любая предельная точка этой последовательности определяет состояние на $\mathbf{A}$

$$
\langle A\rangle=\lim _{N \rightarrow \infty}\left\langle P_{N} A P_{N}\right\rangle_{N}
$$

где $A$ локально.

ТЕОРема 14. Если $\beta$ достаточно мало, то $\log Z_{N} \sim f N$ для некоторой константы $f>0$.

ДокАЗАТЕльство. Заметим сначала, что для $H=0$ это очевидно, так как $\operatorname{Tr}_{H} 1$ $=\operatorname{dim} \mathscr{H}_{N}=2^{N+1}$.

Можно написать

$$
Z_{N}=\sum_{\alpha: R_{0}(\alpha) \leqslant N} z(\alpha), \quad z(\alpha)=\left(e_{\alpha}, \exp \left(-\beta P_{N} H P_{N}\right) e_{\alpha}\right)
$$


или

$$
Z_{N}=\sum_{k=0}^{\infty} \sum_{I_{k}, J_{k}} \sum_{\alpha:|\alpha| \leqslant N} \frac{(-\beta)^{k}}{k !} z\left(\alpha,\left(I_{k}, J_{k}\right)\right),
$$

где для упорядоченных наборов $I_{k}=\left(i_{1}, \ldots, i_{k}\right), J_{k}=\left(j_{1}, \ldots, j_{k}\right)$

$$
z\left(\alpha,\left(I_{k}, J_{k}\right)\right)=\lambda_{i_{1}} \cdots \lambda_{i_{k}}\left(e_{\alpha}, P_{N} a_{i_{k}}\left(j_{k}\right) P_{N} \cdots P_{N} a_{i_{1}}\left(j_{1}\right) P_{N} e_{\alpha}\right),
$$

т.е. $z\left(\alpha,\left(I_{k}, J_{k}\right)\right)$ равен либо $\lambda_{i_{1}} \cdots \lambda_{i_{k}}$, либо 0. Наша цель - получить кластерное представление для

$$
z(N) \doteq \sum_{\alpha:|\alpha|=N} z(\alpha)
$$

вида

$$
z(N)=\sum c_{U_{1}} \cdots c_{U_{n}}
$$

точно определяемое ниже, чтобы применить общую теорию кластерных разложений из [36]. Для вьвода разложения (26) мы используем пересуммирование разложения (25). Для получения (26) нам понадобится индуктивное построение.

В $(26)$ сумма берется по наборам непересекаюшихся подмножеств $\left(U_{1}, \ldots, U_{n}\right)$. Обозначим $U_{0}=\{1, \ldots, N\} \backslash \bigcup_{i=1}^{n} U_{i}$. Мы определим сначала $U_{0}$, а затем "кластеры" $U_{i}$ и числа $c_{U_{i}}$.

Мы сделаем это отдельно для каждого члена

$$
\frac{(-\beta)^{k}}{k !} z\left(\alpha,\left(I_{k}, J_{k}\right)\right)
$$

соответствуюшего слову $\alpha$ длины $N$ и некоторому $\left(I_{k}, J_{k}\right)$, а затем просуммируем результаты.

Определение $U_{0}=U_{0}\left(\alpha,\left(I_{k}, J_{k}\right)\right)$. Скажем, что подстановка $T=T(\operatorname{Sub}, \psi)$ не трогает символ на месте $v$ слова $\alpha$, если образ $\psi$ не содержит $v$. Удобно обозначить $T_{p}$ подстановку, соответствуюшую оператору $b(p)=a_{i_{p}}\left(j_{p}\right), p=1, \ldots, k$. Символ $v$ слова $\alpha$ назьвается нетронутым для данного $\left(I_{k}, J_{k}\right)$, если никакая из подстановок $T_{p}$, $p=1, \ldots, k$, его не трогает. Аналогично, символ $v$ слова $b(s) \ldots b(1) e_{\alpha}$ назьвается нетронутым для данного $\left(I_{k}, J_{k}\right)$, если никакая $T_{l}$ с $s<l \leqslant k$ не трогает его. Для данных $\left(\alpha,\left(I_{k}, J_{k}\right)\right)$ множество $U_{0}=U_{0}\left(\alpha,\left(I_{k}, J_{k}\right)\right)$ определяется как множество всех символов слова $\alpha$, принадлежаших всем словам $b(s) \ldots b(1) e_{\alpha}$ и не тронутых ни для каких $s$.

Определение кластеров $U_{i}\left(\alpha,\left(I_{k}, J_{k}\right)\right)$. Обозначим $\alpha_{0}=\alpha, \alpha_{s}=T_{s} \cdots T_{1} \alpha$, $s \geqslant 1$. Введем систему разбиений $g_{s r}, r, s=0, \ldots, k$, множества символов слова $\alpha_{s}$ на подмножества. Разбиение $g_{0}=g_{0,0}$ определяется как разбиение $\alpha$ на отдельные символы, т.е. разбиение множества $\{1, \ldots, N\}$ на $N$ блоков.

По заданной подстановке $T$, которая переводит слово $\alpha$ в слово $\beta$, и по заданному разбиению $g=g(\alpha)$ множества $V(\alpha)$ символов слова $\alpha$ определим разбиения $g(\beta)$ слова $\beta$. Пусть $V_{1}(\alpha, T)$ - множество символов слова $\alpha$, оставляемых нетронутьми подстановкой $T$. Будем считать, что подстановка $T$ удаляет в новом слове $\beta$ множество символов $V(\alpha) \backslash V_{1}(\alpha)$ и добавляет некоторое множество символов $V_{2}$. Таким образом, $V(\beta)=V_{1}(\alpha) \cup V_{2}$. Назовем разбиение $g(\beta)=g(\beta, g(\alpha), T)$ слова $\beta$ разбиением, 
индуцированным разбиением $g=g(\alpha)$ и подстановкой $T$, если вьполнены следующие условия:

1) если блок $I$ разбиения $g(\alpha)$ принадлежит $V_{1}(\alpha)$, то этот блок остается блоком разбиения $g(\beta)$;

2 ) вершины $V_{2}$ образуют один блок вместе со всеми (не принадлежашими $V_{1}(\alpha)$ ) вершинами всех других блоков $I$ разбиения $g(\alpha)$, пересекаюшимися с $V(\alpha) \backslash$ $V_{1}(\alpha)$.

Определим теперь по индукции разбиения $g_{s}=g_{s, 0}=g_{s}\left(\alpha_{s}\right)$ слов $\alpha_{s}$. Если $g_{s}$ уже определено, то $g_{s+1}$ - разбиение слова $\alpha_{s+1}$, индуцированное подстановкой $T_{s+1}$ и разбиением $g_{s}$ слова $\alpha_{s}$. Далее используем индукцию по $r$. Если разбиение $g_{s+1, r}$ слова $\alpha_{s+1}$ определено, то $g_{s, r+1}$ определяется как разбиение слова $\alpha_{s}$, индуцированное сопряженной подстановкой $T_{s}^{*}$.

Рассмотрим разбиение $g_{0, k}$. Его блоками могут быть отдельные нетронутые символы и другие, состоящие более чем из одного символа. Эти другие обозначим $U_{i}=$ $U_{i}\left(\alpha,\left(I_{k}, J_{k}\right)\right)$ и назовем кластерами относительно заданной пары $\alpha,\left(I_{k}, J_{k}\right)$. Заметим, что число $L=L\left(\alpha,\left(I_{k}, J_{k}\right)\right)$ кластеров не превосходит $k$. Кроме того, множество $\{1, \ldots, k\}$ однозначно разбивается на подмножества $M_{1}=M_{1}\left(\alpha,\left(I_{k}, J_{k}\right)\right), \ldots$, $M_{L}=M_{L}\left(\alpha,\left(I_{k}, J_{k}\right)\right)$ подстановок, относяшихся к одному из кластеров $1, \ldots, L$ соответственно. Пусть $\left(I_{m_{i}}, J_{m_{i}}\right)$, где $I_{m_{i}}=I_{m_{i}}\left(I_{k}, J_{k}\right)$ и $J_{m_{i}}=J_{m_{i}}\left(I_{k}, J_{k}\right)$ - поднабор (с сохраненным порядком) набора $\left(I_{k}, J_{k}\right)$, соответствующий $M_{i}$.

Определение разложения: разложение по связным группам. Рассмотрим некоторое множество $B \subset\{1, \ldots, N\}$. Если ему может соответствовать кластер, то оно является интервалом.

Пусть $M_{1}, \ldots, M_{L}$ - подмножества множества $\{1, \ldots, k\}$ такие, что $\bigcup_{i=1}^{L} M_{i}=$ $\{1, \ldots, k\}$. Обозначим $m_{i}$ мощность множества $M_{i}$. Чтобы получить мультипликативное разложение (26), возьмем сумму всех $\frac{(-\beta)^{k}}{k !} z\left(\alpha,\left(I_{k}^{\prime}, J_{k}^{\prime}\right)\right)$, где наборы $\left(I_{k}^{\prime}, J_{k}^{\prime}\right)$ отличаются от $\left(I_{k}, J_{k}\right)$ лишь порядком членов, причем порядок внутри каждого из множеств $M_{i}$ не меняется. Эта сумма равна

$$
\begin{aligned}
\frac{(-\beta)^{k}}{m_{1} ! \cdots m_{L} !} z\left(\alpha,\left(I_{k}, J_{k}\right)\right) & =\prod_{i=1}^{L} \frac{(-\beta)^{m_{i}}}{m_{i} !} z\left(\alpha,\left(I_{m_{i}}\left(\left(I_{k}, J_{k}\right)\right), J_{m_{i}}\left(\left(I_{k}, J_{k}\right)\right)\right)\right) \\
& =\prod_{i=1}^{L} \frac{(-\beta)^{m_{i}}}{m_{i} !} \prod_{j \in M_{i}} \lambda_{j} .
\end{aligned}
$$

Для определения разложения (26) используем пересуммирование. Для данных подмножеств $U_{0}, U_{1}, \ldots, U_{n}$ рассмотрим все $z\left(\alpha,\left(I_{k}, J_{k}\right)\right)$ такие, что $U_{i}\left(z\left(\alpha,\left(I_{k}, J_{k}\right)\right)\right)=$ $U_{i}, i=0,1, \ldots, n$, и определим для данного связного $U$

$$
c_{U}=\sum \frac{(-\beta)^{k}}{k !} z\left(\alpha,\left(I_{k}, J_{k}\right)\right),
$$

где сумма берется по всем словам $\alpha$ с множеством символов $V(\alpha)=U$ и по наборам $\left(I_{k}, J_{k}\right)$, для которых $U$ является одним связньм кластером. Мы имеем кластерную оценку

$$
c_{U}<c_{1}(C \beta)^{|U|}
$$


Отсюда стандартньй вывод теории кластерных разложений дает $\ln z(N) \sim f N$ для некоторой константы $f>0$. Поэтому

$$
\ln Z_{N} \sim \log \left[z(N)\left(1+\frac{z(N-1)}{z(N)}+\cdots\right] \sim c N\right.
$$

Теорема 15. Существует $\beta_{0}>0$ такое, что для каждого локального А пре$\partial e \Omega$

$$
\langle A\rangle=\lim _{N \rightarrow \infty}\left\langle P_{N} A P_{N}\right\rangle_{N}
$$

существует и аналитичен по $\beta$ для $\beta<\beta_{0}$.

ДокАЗАтельство. Возьмем, например, $A=P_{j, \delta}$-проектор на подпространство, порожденное словами, содержашими слово $\delta$ на месте $j$. Имеем

$$
\begin{aligned}
\operatorname{Tr}_{N}\left[\exp \left(-\beta P_{N} H P_{N}\right) A\right] & =\sum_{k=0}^{\infty} \frac{(-\beta)^{k}}{k !} \operatorname{Tr}_{N}\left(\left(P_{N} H P_{N}\right)^{k} A\right) \\
& =\sum_{k=0}^{\infty} \frac{(-\beta)^{k}}{k !} \sum_{I_{k}, J_{k}} \operatorname{Tr}_{N}\left(a_{i_{k}}\left(j_{k}\right) \ldots a_{i_{1}}\left(j_{1}\right) A\right)
\end{aligned}
$$

где сумма по всем $I_{k}=\left(i_{1}, \ldots, i_{k}\right), J_{k}=\left(j_{1}, \ldots, j_{k}\right)$. Дальнейшее доказательство совершенно аналогично и сводится к применению общей теории кластерных разложений.

ЗАмЕчАнИЕ 5. Случай спинграфов с $p \geqslant 3$ должен быть аналогичен теории, развитой в разделе 4.3 .

5.2. Примеры и структура гамильтонианов. От общей теории мы переходим к простейшим примерам в одномерном случае. Но сначала укажем связь с квантованием Тоофта.

5.2.1. Квантование по Тоофту. Еще Фейнман [49] предполагал, что на планковских длинах физические законы могут иметь вид преобразований конечного множества с дискретным временем. В серии статей [50], [51] Тоофт обсуждает квантование дискретных однозначных (детерминированных) отображений.

Если $f: S \rightarrow S$ - однозначное отображение конечного множества $S$ в себя, то сушествует подмножество $S_{0}$, инвариантное относительно $f$, причем $f: S_{0} \rightarrow S_{0}$ взаимно однозначно. $\mathrm{B} l_{2}\left(S_{0}\right)$ ему соответствует перестановочная матрица $U$, определяемая равенством

$$
(U \phi)(s)=\phi\left(f^{-1} s\right) .
$$

Преобразование $U$ в гильбертовом пространстве $l_{2}(S)$ и считается (по Тоофту) квантованием детерминированного отображения $f$. При этом $U$ эквивалентна прямой сумме циклических сдвигов на дискретных окружностях. Примеры физических систем, для которых возможно такое представление, основаны фактически на унитарной эквивалентности гамильтониана оператору умножения, или (через преобразование Фурье) генератору сдвига. 
Необходимо подчеркнуть, что речь идет как бы о квантовании системы “первого порядка". Тогда существует матрица $H$ такая, что

$$
U_{t}=\exp (i t H), \quad U_{1}=U .
$$

В нецелые моменты времени $U_{t}$ не определяет детерминированного отображения $S$ в себя. Более того, $H$ определена не однозначно, а лишш с точностью до умножения каждого собственного значения на корень определенной степени (длины цикла) из единицы.

Простейший физический пример - частица со спином в магнитном поле: в конечномерном линейном пространстве с базисом $e_{m}, m=-l, \ldots, l$, диагонализация гамильтониана имеет вид $H e_{m}=\mu m e_{m}$. Однако в другом базисе

$$
f_{n}=\frac{1}{\sqrt{N}} \sum_{m} \exp \left(-\frac{2 \pi i m n}{N}\right) e_{m}, \quad n=0,1, \ldots, N-1, \quad N=2 l+1,
$$

в дискретные интервалы времени $t-t^{\prime}=\frac{2 \pi k}{\mu N}$ мы имеем детерминированную динамику $\exp \left(i \frac{2 \pi k}{\mu N} H\right): f_{n} \rightarrow f_{n+k}(\bmod N)$. Тоофт приводит также менее банальные примеры. Однако самые обшие примеры такого рода строятся с помошью квантовых грамматик.

Квантовые грамматики на графрах вводились независимо, но их можно рассматривать и в рамках этих идей Тоофта, но с новыми элементами: операция подстановки на многих граф̆ах неоднозначна (является недетерминированной, в терминологии информатики), множество $S$ счетно (возможен неограниченньй рост). Хотя есть много вариантов, как работать с недетерминированными отображениями, однородность (независимость от места в графе) делает выбор квантования единственным с точностью до технической реализации: последовательная реализация каждого отображения, синхронный параллелизм, асинхронньй параллелизм. Мы выбрали последний вариант. Если же есть несколько детерминированных отображений, то появляется возможность приписьвать им коэффициенты-веса.

5.2.2. Квантовые спиновые системы на фиксированном графе. Если гамильтониан не меняет граф, а только конфигурации, то мы получаем квантовую спиновую систему на этом фиксированном графе. Обратно, любая квантовая спиновая система может быть представлена как грамматика на графе, что может быть достигнуто подбором констант $\lambda_{i}$. В этом случае вложение $\phi_{N}: \mathbf{A}_{N} \rightarrow \mathbf{A}_{N+1}$ задается посредством $A \rightarrow$ $A \otimes 1 \otimes \cdots \otimes 1$. Такие алгебры много изучались, см. [4].

5.2.3. Линейные грамматики и теплищевы операторы. В некоторых случаях получаются интересные примеры $C^{*}$-алгебр, которые ранее возникали из других соображений. Тогда $C^{*}$-алгебра $\mathbf{A}_{N}$, порожденная подстановкой $a \rightarrow a a$, изоморфна $C^{*}$-алгебре теплицевых операторов в $l_{2}\left(\mathbb{Z}_{+}\right)$, порожденной сдвигами. Исследование структуры $C^{*}$-алгебры часто более простая задача, чем исследование спектра гамильтониана, но зачастую уже она дает информацию о спектральном разложении.

Рассмотрим случай, когда $S=\{a, w\}$ состоит из двух букв, а подстановки имеют вид $a w \rightarrow, w \rightarrow a w$. Положим $\lambda(a w \rightarrow w)=\lambda(w \rightarrow a w)=\lambda$. Такая грамматика называется в информатике правой линейной грамматикой. Тогда подпространства 
$\mathscr{H}\left(L_{k}\right)$, где $L_{k}-$ множество слов с точно $k$ символами $w$, инвариантны. Например, $L_{1}=\left\{a^{m} w=a a \ldots a w, m=0,1,2, \ldots\right\}$. Назовем $\mathscr{H}\left(L_{k}\right) k$-частичным пространством. Пусть $H_{k}$ - ограничение $H$ на $\mathscr{H}\left(L_{k}\right)$.

ТЕОремА 16 (одночастичный спектр). $H_{1}$ на $\mathscr{H}\left(L_{1}\right)$ унитарно әквивалентен оператору умножсения на $\lambda\left(z+z^{-1}\right)$ в $L_{2}\left(S^{1}, d \nu\right)$, где $d \nu$ - мера Лебега на единичном круге $S^{1}$ в комплексной плоскости $\mathbb{C}$.

$\mathscr{H}\left(L_{1}\right)$ изоморфно $l_{2}\left(\mathbb{Z}_{+}\right)$посредством $a a \ldots a w \rightarrow m$. При этом изоморфизме $H$ становится теплицевым оператором в $l_{2}\left(\mathbb{Z}_{+}\right)$, равным

$$
\lambda b+\lambda b^{*},
$$

где $b$ - левый сдвиг в $l_{2}\left(\mathbb{Z}_{+}\right)$,

$$
(b f)(m)=f(m-1), \quad m=1,2, \ldots, \quad(b f)(0)=0 .
$$

A $C^{*}$-алгебра $\mathbf{A}$ переходит в $C^{*}$-алгебру $\mathbf{W}_{1}$ теплицевых операторов, т.е. $C^{*}$-алгебру операторов в $l_{2}\left(\mathbb{Z}_{+}\right)$, порожденную $b$. Заметим, что коммутатор $\left[b, b^{*}\right]$ конечномерен. Более общо, имеет место следующая точная последовательность алгебр:

$$
0 \rightarrow K \rightarrow^{j} W_{1} \rightarrow^{\pi} W \rightarrow 0
$$

где $K$ - замкнутьй двусторонний идеал в $W_{1}$, состояший из всех компактных операторов в $l_{2}\left(\mathbb{Z}_{+}\right), j$ - вложение, $\pi$ - естественная проекция $W_{1}$ на $W_{1} / K \sim W$. Действительно, коммутатор любых двух элементов из $W_{1}$ компактен. Последнее легко проверить для мономов $b_{1} b_{2} \ldots b_{k}$, где $b_{i}=b$ или $b^{*}$. Переходом к пределу это доказывается для любых элементов. Отсюда следует, что $W_{1} / K$ коммутативна. Положим

$$
y=\pi b, \quad y^{*}=y^{-1}=\pi b^{*} .
$$

Можно доказать, что элементы

$$
\sum_{i=-k}^{k} c_{i} y^{i}
$$

все различны и что их норма равна $\sum\left|c_{i}\right|$ - достаточно рассмотреть их действие на функции $f(m)$, равные единище для некоторого достаточно большого $m$ и нулю в остальных точках. Отсюда следует, что $W_{1} / K \sim W$. И, значит, непрерьвная часть спектра совпадает со спектром оператора умножения.

Чтобы доказать отсутствие дискретного спектра, перепишем уравнение $A f-\lambda f$ $=\phi$ в виде хорошо известного разностного уравнения

$$
\begin{aligned}
\phi_{n} & =-\lambda f_{n}+f_{n-1}+f_{n+1}, \quad n \geqslant 1, \\
\phi_{0} & =-\lambda f_{0}+f_{1} .
\end{aligned}
$$

Если $\phi_{n} \equiv 0$, то у него нет решений из $l_{2}$. 
Рассмотрим теперь оператор $H$ на всем гильбертовом пространстве, причем мы ограничиваемся только словами с символом $w$ на конце. Каждое такое слово может быть представлено в виде

$$
\alpha=a^{m_{1}} w a^{m_{2}} w \ldots a^{m_{k}} w, \quad m_{1}, m_{2}, \ldots, m_{k}=0,1,2, \ldots
$$

Соответственно, каждьй базисньй вектор в $\mathscr{H}\left(L_{k}\right)$ может быть записан как тензорное произведение

$$
e_{\alpha}=e_{\alpha(1)} \otimes e_{\alpha(1)} \otimes \cdots \otimes e_{\alpha(1)}, \quad \alpha(i)=a^{m_{i}} w
$$

Иными словами, $\mathscr{H}\left(L_{k}\right)$ изоморфно $k$-й тензорной степени $\mathscr{H}\left(L_{1}\right)$, а эволюция имеет вИД

$$
\exp \left(i t H_{1}\right) \otimes \cdots \otimes \exp \left(i t H_{1}\right)
$$

или

$$
H_{k}=H_{1} \otimes 1 \otimes \cdots \otimes 1+\cdots+1 \otimes 1 \otimes \cdots \otimes H_{1} .
$$

Мы доказали следующий результат.

ТЕОРема 17 (многочастичньй спектр). Гильбертово пространство имеет следующее разложсение на инвариантные подпространства:

$$
\mathscr{H}\left(\Sigma^{*}\right)=\bigoplus_{k=0}^{\infty} \mathscr{H}\left(L_{k}\right), \quad \mathscr{H}\left(L_{k}\right)=\mathscr{H}\left(L_{1}\right) \otimes \cdots \otimes \mathscr{H}\left(L_{1}\right)
$$

Гамильтониан $H_{k}$ на $\mathscr{H}\left(L_{k}\right)$ унитарно әквивалентен умножсению на функцию

$$
\sum_{j=1}^{k} \lambda\left(z_{j}+z_{j}^{-1}\right)
$$

в пространстве $L_{2}\left(S^{k}, d \nu\right)$ функций $f\left(z_{1}, \ldots, z_{k}\right)$.

5.2.4. Расширение и сжатие пространства. Пусть алфавит состоит из одного символа $a$. Рассмотрим две подстановки $a \rightarrow a a, a a \rightarrow a, \lambda_{1}=\lambda_{2}=\lambda$, и гамильтониан

$$
H=\lambda \sum_{j=1}^{\infty}\left(a_{1}(j)+a_{2}(j)\right)
$$

с вещественньм $\lambda$. В этом случае гильбертово пространство $\mathscr{H}$ изоморфно $l_{2}\left(\mathbb{Z}_{+}\right)$, так как $a a . . . a$ может быть отождествлено со своей длиной минус 1. Гамильтониан унитарно эквивалентен матрице Якоби

$$
(H f)(n)=\lambda(n-1) f(n-1)+\lambda n f(n+1) .
$$

Назовем этот оператор одночастичным оператором, имея в виду, что "частица" ассоциируется с квантом пространства. Эволюция пространства здесь состоит в (квантовом) расширении и сжатии независимо в каждой точке. 
Найдем обобщенные собственные функции из уравнения

$$
\left(H_{1}-\lambda\right) f=\phi
$$

или

$$
\phi_{n}=n f_{n+1}+(n-1) f_{n-1}-\lambda f_{n} .
$$

Вводя производящие функции

$$
F(z)=\sum_{n=1}^{\infty} f_{n} z^{n}, \quad \Phi(z)=\sum_{n=1}^{\infty} \phi_{n} z^{n},
$$

получим для $F(z)$ следуюшее уравнение:

$$
F^{\prime}-\frac{\lambda+\frac{1}{z}}{1+z^{2}} F=\frac{\Phi}{1+z^{2}}
$$

Однородное уравнение имеет следуюшее решение:

$$
\exp \left(\int \frac{\lambda+\frac{1}{z}}{1+z^{2}} d z\right)=z\left(\frac{z+i}{z-i}\right)^{\frac{1}{2}+\frac{i \lambda}{2}} .
$$

Отсюда следует, например, отсутствие дискретного спектра. Более подробно эта модель исследована в [52].

5.2.5. Лоренщевы модели и грамматики. Лоренщева модель, см. [53], [54], определяется так. Граф строится следуюшим образом. На цилиндре $\mathbb{R} \times S^{1}=\{(x, t)\}$ на каждой из прямых (слоев) $t=0, \ldots, N$ имеется $l(t)$ точек $x_{1}(t), \ldots, x_{l(t)}(t)$, следуюших друг за другом по часовой стрелке. Каждая пара соседей соединена ребром на прямой $t=$ const. Каждая точка $x_{i}(t)$ соединена ребрами с $k(i, t)=1,2, \ldots$ идушими подряд точками слоя $t+1$, пусть $K(i, t+1)$ - множество этих точек. Предполагается, что $K(i, t+1) \cap K(i+1, t+1)$ состоит точно из одной точки, крайней для каждого из них, соответственно справа и слева. Нумерация точек неважна, граф рассматривается с точностью до изоморфизма, сохраняющего слои. Каждому графу $G$ ставится в соответствие "амплитуда"

$$
z(G)=\exp \left(\lambda \sum_{i, t} k(i, t)\right)
$$

Заметим, что $\sum_{i} k(i, t)$ равно числу треугольников между слоями $t$ и $t+1$. В то же время $\sum_{i, t} k(i, t)=2 \sum_{t} l(t)$.

Здесь $k_{i}$ - произвольные положительные числа, и унитарность этого преобразования явно не просматривается. Если же $k_{i} \leqslant$ const, то можно построить унитарньй и евклидов аналоги данного преобразования.

Унитарный аналог совпадает с построенной квантовой грамматикой, расширением и сжатием пространства. Действительно, рассмотрим в момент 0 триангуляцию диска с $m$ ребрами на гранище. Последовательность ребер на границе отождествим со словом $a a \ldots a$ длины $m$. Действие оператора, отвечающего подстановке $a \rightarrow a a$, 
состоит в прикреплении треугольника (имеющего два других ребра вовне диска) к соответствующему ребру границы, а действие оператора, отвечающего подстановке $a a \rightarrow a$, состоит в прикреплении треугольника к двум последовательным ребрам границы так, чтобы одно ребро было вовне диска.

Случайный аналог этой квантовой грамматики был изучен в [11]. Было показано, что получается другой универсальньй класс. Чтобы возник класс, соответствующий динамическим триангулящиям с экспонентой $\frac{7}{2}$, надо рассматривать немарковскую динамику с квадратичньм преобразованием мер.

Лоренщевы модели как гиббсовские семейства подробно изучались в [52]. Важной для физики является связь таких моделей с причинными множествами, см. обзоры [45], [53].

5.2.6. Частища на квантовом одномерном пространстве. Рассмотрим грамматику с $S=\{a, w\}$ и подстановками

$$
a \rightarrow a a, \quad a a \rightarrow a, \quad a w \rightarrow w a, \quad w a \rightarrow a w
$$

Подпространство $\mathscr{H}_{1}$, порожденное словами $a^{k} w a^{l}, k, l=0,1,2, \ldots$, с одним символом $w$, инвариантно. Гамильтониан является смесью $H$, определенного вьше, и дискретного лапласиана $H_{1}$ для свободной шредингеровской частицы в $l_{2}$ на конечном множестве.

5.2.7. Два типа квантов пространства. Пусть $S=\{a, b\}$, рассмотрим четыре подстановки

$$
\text { 1) } a \rightarrow a a, \quad 2) \quad a a \rightarrow a, \quad 3) \quad b \rightarrow b b, \quad \text { 4) } b b \rightarrow b
$$

Здесь есть два инвариантных одночастичных пространства $\mathscr{H}_{a}, \mathscr{H}_{b}$. Например, $\mathscr{H}_{a}$ порождается словами $a, a^{2}=a a, \ldots, a^{n}, \ldots$ Есть два инвариантных двухчастичных пространства $\mathscr{H}_{a b}=\mathscr{H}_{a} \otimes \mathscr{H}_{b}, \mathscr{H}_{b a}=\mathscr{H}_{b} \otimes \mathscr{H}_{a}$. Например, $\mathscr{H}_{a b}$ порождено словами $a^{k} b^{l}, k, l>0$. В общем случае для каждого $n$ существует два инвариантных $2 n$-частичных пространства: $\mathscr{H}_{(a b)_{n}}$, порожденное словами $a^{k_{1}} b^{l_{1}} \ldots a^{k_{n}} b^{l_{n}}$, $\mathscr{H}_{(a b)_{n}}=\mathscr{H}_{a} \otimes \mathscr{H}_{b} \otimes \cdots \otimes \mathscr{H}_{a} \otimes \mathscr{H}_{b}$, и $\mathscr{H}_{(b a)_{n}}$, определяемое аналогично. Так же для любого $n$ существует два инвариантных $(2 n+1)$-частичных пространства $\mathscr{H}_{b(a b)^{n}}=\mathscr{H}_{b} \otimes \mathscr{H}_{a} \otimes \mathscr{H}_{b} \otimes \cdots \otimes \mathscr{H}_{a} \otimes \mathscr{H}_{b}$ и $\mathscr{H}_{(a b)^{n} a}$, определяемое аналогично. Спектр гамильтониана здесь сводится, ввиду разложения

$\mathscr{H}=\mathscr{H}_{0} \oplus \mathscr{H}_{a} \oplus \mathscr{H}_{b} \oplus \mathscr{H}_{a b} \oplus \mathscr{H}_{b a} \oplus \cdots \oplus \mathscr{H}_{(a b)_{n}} \oplus \mathscr{H}_{(b a)_{n}} \oplus \mathscr{H}_{b(a b)^{n}} \oplus \mathscr{H}_{(a b)^{n} a} \cdots$,

к спектру двух одночастичных гамильтонианов, о которых речь шла вьше. Например, на $\mathscr{H}_{a b}$ эволющия задается гамильтонианом $\mathscr{H}_{a} \otimes 1+1 \otimes \mathscr{H}_{b}$.

5.2.8. Симметрии и квантовые грамматики. Здесь мы отметим, что с данной квантовой грамматикой связаны некоторые представления алгебры Ли $\mathscr{D}$ диффеоморфизмов окружности. Обозначим $D_{n}, D_{-n}$ - гамильтонианы, отвечаюшие подстановкам $a \rightarrow a^{n+1}$ и $a^{n+1} \rightarrow a, n \geqslant 0$, соответственно. Тогда в базисе $\nu_{k}=e_{a^{k}}$ имеем, например, $D_{n} \nu_{k}=k \nu_{k+n}, n \geqslant 0$. Однако при $n<0$ мы имеем только $D_{n} \nu_{k}=(k+n+1) \nu_{k+n}$. Для больших $k$ и фиксированных $m, n$ можно доказать, что

$$
\left(D_{m} D_{n}-D_{n} D_{m}\right) \nu_{k}=(m-n+o(1)) D_{n+m} \nu_{k}
$$


В этом случае мы скажем, что задано асимптотическое представление алгебры $\mathscr{D}$. Мы получаем больше для замкнутых слов (периодические граничные условия). Для них соотношение $D_{n} \nu_{k}=k \nu_{k+n}$ имеет место для всех $|n|<k$, и, значит, соотношение

$$
\left(D_{m} D_{n}-D_{n} D_{m}\right) \nu_{k}=(m-n) D_{n+m} \nu_{k}
$$

имеет место для всех $|n|,|m| \ll k$. Иначе говоря, последнее соотношение имеет место для всех $m, n$ везде, кроме конечномерного подпространства $\mathscr{H}(m, n)$. Заметим, что в обоих случаях спектр $D_{0}$ совпадает с $\mathbb{Z}_{+}$.

Чтобы получить точное представление алгебры $\mathscr{D}$, надо рассматривать замкнутые слова из двух символов $a, a^{-1}$ с соотношениями $a a^{-1}=a^{-1} a=e$, где $e-$ пустое слово, и подстановки $D_{n}: a \rightarrow a^{n+1}, a^{-1} \rightarrow a^{n+1}$ для всех $n \in \mathbb{Z}$. Заметим, что речь идет о представлении $-V_{0,0}$ в обозначениях [55].

Рассмотрим линейные циклические графы (т.е. слова с периодическими граничными условиями) и грамматику на них с подстановками

$$
a \rightarrow a a, \quad a a \rightarrow a, \quad a w \rightarrow w a, \quad w a \rightarrow a w .
$$

Выделим среди них слова с одним символом $w$. Заметим, что все замкну тые слова одинаковой длины с единственньм символом $w$ эквивалентны. Порожденное ими гильбертово пространство обозначим $\mathscr{H}_{1}$, оно изоморфно $l_{2}\left(\mathbb{Z}_{+}\right)$. Заметим, что в $\mathscr{H}_{1}$ действует асимптотическое представление алгебры Вирасоро.

Если перейти от классов эквивалентности замкнутых слов к занумерованным словам, т.е. к базису $e_{k} \otimes j, j \in \mathbb{Z}_{k}$, то будет определено действие групшы сдвигов $\mathbb{Z}:$ на каждом $-e_{k} \otimes j \rightarrow e_{k} \otimes(j+n), n \in \mathbb{Z}$, где $j+n$ берется по модулю $k$. Таким образом, симметрия (ранее тривиальная) проявляется в добавленной нумерации. Действие $\mathbb{Z}$ можно представить с помощью фермионных переменных в соответствии с обсужденным выше квантованием Тоофта, см. [50], [51].

\section{СПИСОК ЛИТЕРАТУРЫ}

[1] Р.Л.Добрушин. Описание случайного поля при помощи условных вероятностей и условия его регулярности // Теория вероятн. и ее примен. 1968. Т. 13. № 2. С. 201-229.

[2] O. Lanford, D. Ruelle. Observables at infinity and states with short range correlations in statistical mechanics // Comm. Math. Phys. 1969. V. 13. P. 174-215.

[3] Дж. Глимм, А. Джаффе. Математические методы квантовой физики. М.: Мир, 1984.

[4] O. Brattelli, D. Robinson. Operator Algebras and Quantum Statistical Mechanics. I, II. New York: Springer-Verlag, 1979, 1981.

[5] A. Connes. A short survey of noncommutative geometry // J. Math. Phys. 2000. V. 41. P. $3832-3866$.

[6] G. Landi. An Introduction to Noncommutative Spaces and their Geometries. Berlin: Springer-Verlag, 1997. (Lecture Notes in Phys. V. 51.)

[7] G. Landi, F. Lizzi. Projective systems of noncommutative lattices as a pregeometric substratum // Quantum Groups and Fundamental Physical Applications / ed. D. Kastler, M. Rosso. Nova Science Publishers, USA, 1998.

[8] Yu. Manin. Topics in Noncommutative Geometry. Princeton: Princeton Univ. Press, 1991.

[9] R. Penrose. Angular momentum: an approach to combinatorial space-time // Quantum Theory and Beoynd / ed. T. Bastin. Cambridge: Cambridge Univ. Press, 1971. P. 151-180.

[10] J. Baez. Spin network states in gauge theory // Adv. Math. 1996. V. 117. P. 253-272. 
[11] В. Малышев. Вероятность вокруг квантовой гравитации // УМН. 1999. Т. 54. № 4. C. $3-46$.

[12] J. Ambjorn, M. Carfora, A. Marzuoli. The geometry of dynamical triangulations // http://xxx.lanl.gov/hep-th/9612069.

[13] D. V. Boulatov. Three-dimensional simplicial gravity and combinatorics of group presentations // J. High Energy Phys. 1998. V. 10. № 7.

[14] E. Bender, E. Canfield, L. Richmond. The asymptotic number of rooted maps on a surface. II. Enumeration by vertices and faces // J. Combin. Theory Ser. A. 1993. V. 63. P. $318-329$.

[15] М. Крикун, В. Малышев. Асимптотическое число карт на компактных ориентированных поверхностях // Дискретн. матем. 2001. Т. 13. № 2. С. 89-98.

[16] T. Walsh, A. Lehman. Counting rooted maps by genus. I-III // J. Combin. Theory Ser. B. 1972. V. 13. P. 192-218; 1972. V. 13. P. 122-141; 1975. V. 18. P. 222-259.

[17] P. Bialas, Z. Burda, D. Johnston. Phase diagram of the mean-field model of simplicial gravity // Nuclear Phys. B. 1999. V. 542. P. 413-424.

[18] G. Thorleifson. Lattice gravity and random surfaces // Nuclear Phys. B. Proc. Suppl. 1999. V. 73. P. 133-145.

[19] V. Malyshev, A. Yakovlev. Condensation in large closed Jackson networks // Ann. Appl. Probab. 1996. V. 6. №1. P. 92-115.

[20] B. Bollobas. Random Graphs. London: Academic Press, 1985.

[21] I. Goulden, D. Jackson. Combinatorial Enumeration. New York: Wiley, 1983.

[22] V. Kazakov, M. Staudacher, Th. Wynter. Exact solution of discrete two-dimensional $R^{2}$ gravity // Nuclear Phys. B. 1996. V. 471. P. 309-333.

[23] М. Атья. Геометрия и физика узлов. М.: Мир, 1995.

[24] Y. Watabiki. Construction of noncritical string field theory by transfer matrix formalism in dynamical triangulation // Nuclear Phys. B. 1995. V. 441. № 1-2. P. 119-163.

[25] J. Baez. Higher-dimensional algebra and Planck-scale physics // http://xxx.lanl.gov/gr-qc /9902017.

[26] N. Ishibashi, H. Kawai. String field theory of noncritical strings // Phys. Lett. B. 1994. V. 322. P. $67-78$.

[27] J. Ambjorn, B. Durhuus, T. Jonsson. Quantum Geometry. Cambridge, 1997.

[28] W. Tutte. The enumerative theory of planar maps // A Survey of Combinatorial Theory / ed. J. Srivastava et al. Amsterdam: North-Holland, 1973. P. 437-448.

[29] W. Magnus, A. Karrass, D. Solitar. Combinatorial Group Theory. New York: Wiley, 1966.

[30] В. Мальшев. Макроразмерность - инвариант локальной динамики // Теория вероятн. и ее примен. 2000. Т. 45. № 2. С. 368-374.

[31] Ph. Gibbs. The small scale structure of space-time: A bibliographical Review // Preprint, 1995, hep-th/9506171.

[32] D. Wallace. The quantization of gravity - an introduction // Preprint, 2000, gr-qc/0004005.

[33] C. Rovelli. Strings, loops and others: a critical survey of the present approaches to quantum gravity // Preprint, 1998, gr-qc/9803024.

[34] J. Frohlich. Regge calculus and discretized gravitational functional integral // Preprint. Zürich, 1990 .

[35] L. A. Pastur. Spectral and probabilistic aspects of random matrix models // Algebraic and Geometric Methods in Mathematical Physics / ed. A. Boutet de Monvel, V. A. Marchenko. Dordrecht: Kluwer, 1996. P. 207-242. (Math. Phys. Stud. V. 19.)

[36] В. А. Малышев, Р. А. Минлос. Гиббсовские случайные поля. М.: Наука, 1985.

[37] J. Harer, D. Zagier. The Euler characteristic of the moduli space of curves // Invent. Math. 1986. V. 85. P. 457-485.

[38] M. Kontsevich. Intersection theory on the moduli spaces of curves and the matrix Airy function // Comm. Math. Phys. 1992. V. 147. №1. P. 1-23.

[39] A. Okounkov. Gromov-Witten theory, Hurwitz numbers, and matrix models. I // E-print archive, 2001, math.AG/0101147. 
[40] A. Zvonkin. Matrix integrals and map enumeration: An accessible introduction // Math. Comput. Modelling. 1997. V. 26. № 8-10. P. 281-304.

[41] E. Brezin, C. Itzykson, G. Parisi, J. Zuber. Planar Diagrams // Comm. Math. Phys. 1978. V. 59. P. 35-47.

[42] V. A. Kazakov. Solvable matrix models. Talk delivered at Workshop "Matrix Models and Painleve equations", Berkeley, 1999 // E-print archive, 2000, hep-th/0003064.

[43] M. Reisenbereger, C. Rovelli. Spin foams as Feynman diagrams // ArXiv:gr-qc/000 2083

[44] E. Witten. 2+1 gravity as an exactly soluble model // Nuclear Phys. B. 1988. V. 311. P. $46-78$.

[45] D. Reid. Introduction to causal sets: an alternate view of space-time structure // http://xxx.lanl.gov/gr-qc/9909075.

[46] G. Landi. An introduction to noncommutative spaces and their geometry // E-print archive, 1997, hep-th/0003064.

[47] М. Рид, Б. Саймон. Методы современной математической физики. Т. 2. М.: Мир, 1978.

[48] V. Malyshev. Quantum Grammars // J. Math. Phys. 2000. V. 7. P. 4508-4520.

[49] R. Feynman. Simulating physics with computers // Internat. J. Theoret. Phys. 1982. V. 21. P. $467-488$.

[50] G. 't Hooft. Quantization of discrete deterministic theories by Hilbert space extension // Nuclear Phys. B. 1990. V. 342. P. 471-485.

[51] G. 't Hooft. Determinism and dissipation in quantum gravity // Preprint, 2000, http://xxx.lanl.gov/hep-th/0003005.

[52] V. Malyshev, A. Yambartsev, A. Zamyatin. Unitary and completely-positive Lorentzian models // Moscow Math. J. 2001. V. 1. №3.

[53] J. Ambjoern, J. Jurkiewich, R. Loll. Lorentzian and Euclidean quantum gravity analytical and numerical results // http://xxx.lanl.gov/hep-th/0001124.

[54] J. Ambjoern, R. Loll. Non-perturbative Lorentzian quantum gravity, causality and topology change // Nuclear Phys. B. 1999. V. 536. № 1-2. P. 407-434.

[55] V. G. Kac, A. K. Raina. Bombay Lectures on Highest Weight Representations of Infinite Dimensional Lie Algebras. Singapore: World Scientific, 1987. (Adv. Ser. Math. Phys. V. 2.)

Московский государственньй

Поступила в редакцию

университет им. М. В. Ломоносова

17.11 .2000 